\title{
Pacific
}

Journal of

Mathematics

\section{CLASSIFICATION OF COHOMOGENEITY ONE MANIFOLDS IN LOW DIMENSIONS}

COREy A. Hoelscher 


\title{
CLASSIFICATION OF COHOMOGENEITY ONE MANIFOLDS IN LOW DIMENSIONS
}

\author{
COREy A. HoElscher
}

\begin{abstract}
A cohomogeneity one manifold is a manifold whose quotient by the action of a compact Lie group is one-dimensional. Such manifolds are of interest in Riemannian geometry in the context of nonnegative sectional curvature, as well as in other areas of geometry and in physics. We classify compact simply connected cohomogeneity one manifolds in dimensions 5, 6 and 7. We also show that all such manifolds admit metrics of nonnegative sectional curvature, with the possible exception of two families of manifolds.
\end{abstract}

\section{Introduction}

Manifolds with nonnegative curvature play a special role in Riemannian geometry, but finding new examples is particularly difficult. Recently, Grove and Ziller [2000] constructed a large class of nonnegatively curved metrics on certain cohomogeneity one manifolds, that is, manifolds with an action by a compact Lie group whose orbit space is one-dimensional. In particular, they showed that all principal $S^{3}$ bundles over $S^{4}$ can be written as cohomogeneity one manifolds with metrics of nonnegative sectional curvature. They also showed that every compact cohomogeneity one manifold admits a metric of nonnegative Ricci curvature and admits a metric of positive Ricci curvature if and only if its fundamental group is finite. So cohomogeneity one manifolds provide a good setting for examples of manifolds with certain curvature restrictions. Cohomogeneity one actions are of independent interest in the field of group actions since they are the simplest examples of inhomogeneous actions. They also arise in physics as new examples of Einstein and EinsteinSasaki manifolds [Conti 2007, Gibbons et al. 2004, Gauntlett et al. 2004] and as manifolds with $G_{2}$ and Spin(7)-holonomy [Cleyton and Swann 2002, Cvetič et al. 2004, Reidegeld 2009]. It is then interesting to ask how big the class of cohomogeneity one manifolds is. Such manifolds where classified in dimensions four and lower in [Parker 1986] and [Neumann 1968], and some partial results for dimension eight can be found in [Gambioli 2008]. Physicists are interested in those of dimension 5, 7 and 8, and many of the most interesting examples appearing in

MSC2000: 53C20, 57S15.

Keywords: cohomogeneity one manifolds, nonnegative sectional curvature. 
[Grove and Ziller 2000] were 7-dimensional. This paper mainly classifies compact simply connected cohomogeneity one manifolds in dimensions 5, 6 and 7.

Before we state the theorem we will review some facts about cohomogeneity one manifolds. A compact cohomogeneity one manifold with finite fundamental group has a description in terms of its group diagram

$$
G \supset K^{-}, K^{+} \supset H
$$

where $G$ is the group that acts, that is assumed to be compact, $H$ is a principal isotropy subgroup, and $K^{ \pm}$are certain nonprincipal isotropy subgroups that both contain $H$; see Section 1.1 for details. We will henceforth describe actions in terms of their corresponding group diagrams.

If the group $G$ is disconnected then the identity component still acts by cohomogeneity one. Further, since the isometry group of a compact Riemannian manifold is a compact Lie group, it is natural to restrict our attention to actions by compact groups. So we will always assume that $G$ is compact and connected.

Cohomogeneity one actions can be easily built from lower-dimensional actions by taking products. Say $G_{1}$ acts by cohomogeneity one on $M_{1}$, and $G_{2}$ acts transitively on $M_{2}$. Then it is clear that $G_{1} \times G_{2}$ acts by cohomogeneity one on $M_{1} \times M_{2}$, as a product. Such actions are referred to as product actions.

We call a cohomogeneity one action of $G$ on $M$ reducible if there is a proper normal subgroup of $G$ that still acts by cohomogeneity one with the same orbits. This gives a way of reducing these actions to simpler actions. Conversely, there is a natural way of extending an arbitrary cohomogeneity one action to an action by a possibly larger group. Such extensions, called normal extensions, are described in more detail in Section 1.11. It turns out that every reducible action is a normal extension of its restricted action. Therefore it is natural to restrict ourselves to nonreducible actions in the classification.

Recall that a cohomogeneity one action is nonprimitive if all the isotropy subgroups, $\mathrm{K}^{-}, \mathrm{K}^{+}$and $H$ for some group diagram representation, are all contained in some proper subgroup $L$ in $G$. Such a nonprimitive action is well known to be equivalent to the usual $G$ action on $G \times_{L} M_{L}$, where $M_{L}$ is the cohomogeneity one manifold given by the group diagram $L \supset K^{-}, K^{+} \supset H$. With these definitions in place, we are ready to state the main result.

Theorem A. Every nonreducible cohomogeneity one action on a compact simply connected manifold of dimension 5, 6 or 7 by a compact connected group is equivalent to one of the following:

(i) an isometric action on a symmetric space,

(ii) a product action,

(iii) the $\mathrm{SO}(2) \mathrm{SO}(n)$ action on the Brieskorn variety $B_{d}^{2 n-1}$, 
(iv) one of the primitive actions listed in Table I or a nonprimitive action from Table II.

Hence every cohomogeneity one action on such a manifold by a compact connected group is a normal extension of one of these actions.

Remark. When reading the tables below, we observe the following conventions and notations. In all cases we denote $H_{ \pm}:=H \cap K_{0}^{ \pm}$. Here $H_{+}$is either $H_{0}$ in the case that $\operatorname{dim} K^{+} / H>1$, or $H_{0} \cdot \mathbb{Z}_{n}$ for some $n \in \mathbb{Z}$ in the case $\operatorname{dim} K^{+} / H=1$, and similarly for $H_{-}$. Here and throughout, $L_{0}$ denotes the identity component of a given Lie group $L$. Next, we always know that $H \subset K^{ \pm}$, and this puts some unstated restrictions on the groups in the tables. We understand $H_{0}$ to be trivial unless otherwise stated. In the tables, we also assume, when we have a group of the form $\left\{\left(e^{i p \theta}, e^{i q \theta}\right)\right\}$, that $\operatorname{gcd}(p, q)=1$, and similarly for other such groups. Furthermore $\theta, \phi \in \mathbb{R}$ and $z, w \in S^{1} \subset \mathbb{C} \subset \mathbb{W}$ are taken to vary arbitrarily, while integers $a, b, c, m, n, p, q, r, s, \lambda, \mu$ are understood to be fixed within a given group diagram. Finally, $i, j, k \in S^{3} \subset \mathbb{W}$ are the usual unit quaternions.

Notice that many of the diagrams are not effective, since $G$ and $H$ share a finite normal subgroup. We have allowed this possibility so that our descriptions are simpler. The effective version of each action can always be determined by quotienting each group in the diagram by $Z(G) \cap H$, where $Z(G)$ is the center of $G$. See Section 1.1 for details.

In Section 5.3, we collect some facts about each family of actions in Tables I and II. This section would be of interest to the reader who wants to quickly know what can be easily said about these actions. For example some of these actions are of types (i), (ii) and (iii) of Theorem A for special choices of parameters. In fact, it has since been shown in [Hoelscher 2010a] that all actions of type $N_{A}^{6}$ are isometric actions on $S^{3} \times S^{3}$ and hence this entry could have been left out of Table II. See Section 5.3 for more details. We also describe the isometric actions on symmetric spaces from Theorem A in Section 5.1 and the Brieskorn actions in Section 5.2.

The next theorem addresses nonnegative sectional curvature. Verdiani [2004] and Grove, Wilking, and Ziller [2008] classified simply connected cohomogeneity one manifolds admitting invariant metrics of positive sectional curvature. Since it is very difficult to distinguish between manifolds admitting positive curvature and those that merely admit nonnegative curvature, it is interesting to see which cohomogeneity one manifolds admit invariant metrics of nonnegative curvature.

One particularly interesting example in this context is the Brieskorn variety $B_{d}^{2 n-1}$ with the cohomogeneity one action by $\mathrm{SO}(n) \mathrm{SO}(2)$; see Section 5.2. In [Grove et al. 2006], it was shown that $B_{d}^{2 n-1}$ admits an invariant metric of nonnegative sectional curvature if and only if $n \leq 3$ or $d \leq 2$. So most of these actions do not admit invariant nonnegatively curved metrics. 


\begin{tabular}{|c|c|}
\hline$P^{5}$ & $S^{3} \times S^{1} \supset\left\{\left(e^{i \theta}, 1\right)\right\} \cdot H,\left\{\left(e^{j p \theta}, e^{i \theta}\right)\right\} \supset\langle(j, i)\rangle$ \\
\hline$P_{A}^{7}$ & $\begin{aligned} S^{3} \times S^{3} \supset\left\{\left(e^{i p_{-} \theta}, e^{i q_{-} \theta}\right)\right\},\left\{\left(e^{j p_{+} \theta}, e^{j q_{+} \theta}\right)\right\} \cdot H & \supset\langle(i, i)\rangle, \\
& \text { where } p_{-}, q_{-} \equiv 1 \bmod 4\end{aligned}$ \\
\hline$P_{B}^{7}$ & $\begin{array}{r}S^{3} \times S^{3} \supset\left\{\left(e^{i p_{-} \theta}, e^{i q_{-} \theta}\right)\right\} \cdot H,\left\{\left(e^{j p_{+} \theta}, e^{j q_{+} \theta}\right)\right\} \cdot H \supset\langle(i, i),(1,-1)\rangle, \\
\text { where } p_{-}, q_{-} \equiv 1 \bmod 4, p_{+} \text {even }\end{array}$ \\
\hline$P_{C}^{7}$ & $\begin{array}{r}S^{3} \times S^{3} \supset\left\{\left(e^{i p_{-} \theta}, e^{i q_{-} \theta}\right)\right\} \cdot H,\left\{\left(e^{j p_{+} \theta}, e^{j q_{+} \theta}\right)\right\} \cdot H \supset \Delta Q, \\
\text { where } p_{ \pm}, q_{ \pm} \equiv 1 \bmod 4\end{array}$ \\
\hline$P_{D}^{7}$ & $\begin{array}{l}\beta^{3} \times S^{3} \supset\left\{\left(e^{i p \theta}, e^{i q \theta}\right)\right\}, \Delta S^{3} \cdot \mathbb{Z}_{n} \supset \mathbb{Z}_{n}, \\
\quad \text { where } n=2 \text { and } p \text { or } q \text { even; or } n=1 \text { and } p \text { and } q \text { arbitrary }\end{array}$ \\
\hline
\end{tabular}

Table I. Primitive cohomogeneity one manifolds of Theorem A

On the other hand, Grove and Ziller [2000] described a construction for metrics of nonnegative sectional curvature on a large class of cohomogeneity one manifolds. They showed that every cohomogeneity one manifold with two nonprincipal orbits of codimension 2 admits an invariant metric of nonnegative sectional curvature. The following theorem relies heavily on that result.

Theorem B. Every nonreducible cohomogeneity one action of a compact connected group on a compact simply connected manifold of dimension 7 or less admits an invariant metric of nonnegative sectional curvature, except the Brieskorn variety $B_{d}^{7}$ for $d \geq 3$, and possibly some of the members of the family

$$
S^{3} \times S^{3} \supset\left\{\left(e^{i p \theta}, e^{i q \theta}\right)\right\}, \Delta S^{3} \cdot \mathbb{Z}_{n} \supset \mathbb{Z}_{n}
$$

of actions, where $(p, q)=1$ and either $n=1$, or else $p$ or $q$ is even and $n=2$.

Remark. In the case $n=2$ and $q=p+1$, these actions are isometric actions on certain positively curved Eschenburg spaces ([Ziller 1998] or [Grove et al. 2008]). So in fact, many of the members of this exceptional family are already known to admit invariant metrics of positive sectional curvature. It is then reasonable to expect many more of them to admit nonnegative curvature as well.

Determining the full topology of all the manifolds appearing in the classification above is a difficult problem. However, in dimension 5 we can give a complete answer here.

Theorem C. Every compact simply connected cohomogeneity one manifold of dimension 5 must be diffeomorphic to $S^{5}$, $\mathrm{SU}(3) / \mathrm{SO}(3)$, or one of the two $S^{3}$ bundles over $S^{2}$. 


\begin{tabular}{|c|c|}
\hline$N^{5}$ & $\begin{array}{c}S^{3} \times S^{1} \supset\left\{\left(e^{i p_{-} \theta}, e^{i q_{-} \theta}\right)\right\} \cdot H,\left\{\left(e^{i p_{+} \theta}, e^{i q_{+} \theta}\right)\right\} \cdot H \supset H_{-} \cdot H_{+}, \\
\text {where } K^{-} \neq K^{+}, \quad\left(q_{-}, q_{+}\right) \neq \mathbf{0}, \quad \operatorname{gcd}\left(q_{-}, q_{+}, d\right)=1, \\
d=\#\left(K_{0}^{-} \cap K_{0}^{+}\right) / \#\left(H \cap K_{0}^{-} \cap K_{0}^{+}\right)\end{array}$ \\
\hline & $\begin{array}{cc}3 \times T^{2} \supset\left\{\left(e^{i a_{-} \theta}, e^{i b_{-} \theta}, e^{i c_{-} \theta}\right)\right\} \cdot H,\left\{\left(e^{i a_{+} \theta}, e^{i b_{+} \theta}, e^{i c_{+} \theta}\right)\right\} \cdot H \supset H \\
\text { where } K^{-} \neq K^{+}, \quad H=H_{-} \cdot H_{+}, & \operatorname{gcd}\left(b_{ \pm}, c_{ \pm}\right)=1, \\
a_{ \pm}=r b_{ \pm}+s c_{ \pm}, & K_{0}^{-} \cap K_{0}^{+} \subset H\end{array}$ \\
\hline $\begin{array}{l}6 \\
B\end{array}$ & ${ }^{3} \times S^{3} \supset\left\{\left(e^{i \theta}, e^{i \phi}\right)\right\},\left\{\left(e^{i \theta}, e^{i \phi}\right)\right\} \supset\left\{\left(e^{i p \theta}, e^{i q \theta}\right)\right\} \cdot \mathbb{Z}_{n}$ \\
\hline${ }_{C}$ & $\times S^{3} \supset T^{2}, S^{3} \times \mathbb{Z}_{n} \supset S^{1} \times \mathbb{Z}_{n}$ \\
\hline & $\times S^{3} \supset T^{2}, S^{3} \times S^{1} \supset\left\{\left(e^{i p \theta}, e^{i \theta}\right)\right\}$ \\
\hline$E$ & $\times S^{3} \supset S^{3} \times S^{1}, S^{3} \times S^{1} \supset\left\{\left(e^{i p \theta}, e^{i \theta}\right)\right\}$ \\
\hline$F$ & $\mathrm{~J}(3) \supset \mathrm{S}(\mathrm{U}(2) \mathrm{U}(1)), \mathrm{S}(\mathrm{U}(2) \mathrm{U}(1)) \supset \mathrm{SU}(2) \mathrm{SU}(1) \cdot \mathbb{Z}_{n}$ \\
\hline$V_{A}^{7}$ & $\times S^{3} \supset\left\{\left(e^{i p_{-} \theta}, e^{i q_{-} \theta}\right)\right\} \cdot H_{+},\left\{\left(e^{i p_{+} \theta}, e^{i q_{+} \theta}\right)\right\} \cdot H_{-} \supset H_{-} \cdot H_{+}$ \\
\hline$V_{B}^{7}$ & $\begin{aligned} & \times S^{3} \supset\left\{\left(e^{i p \theta}, e^{i q \theta}\right)\right\} \cdot H_{+},\left\{\left(e^{j \theta}, 1\right)\right\} \cdot H_{-} \supset H_{-} \cdot H_{+} \\
& \text {where } H_{ \pm}=\mathbb{Z}_{n_{ \pm}} \subset K_{0}^{ \pm}, n_{+} \leq 2,4 \mid n_{-}, p_{-} \equiv \pm n_{-} / 4 \bmod n_{-}\end{aligned}$ \\
\hline$V_{C}^{7}$ & $\begin{aligned} 3 & \times S^{3} \supset\left\{\left(e^{i p \theta}, e^{i q \theta}\right)\right\}, S^{3} \times \mathbb{Z}_{n} \supset H \\
& \text { where }(q, n)=1 \text { and } \mathbb{Z}_{n} \simeq H \subset\left\{\left(e^{i p \theta}, e^{i q \theta}\right)\right\}\end{aligned}$ \\
\hline $\mathrm{V}_{D}^{7}$ & $\begin{array}{r}3 \times S^{3} \times S^{1} \supset\left\{\left(z^{p} w^{\lambda m}, z^{q} w^{\mu m}, w\right)\right\},\left\{\left(z^{p} w^{\lambda m}, z^{q} w^{\mu m}, w\right)\right\} \supset H_{0} \cdot \mathbb{Z}_{n} \\
\quad \text { where } H_{0}=\left\{\left(z^{p}, z^{q}, 1\right)\right\}, p \mu-q \lambda=1 \text { and } \mathbb{Z}_{n} \subset\left\{\left(w^{\lambda m}, w^{\mu m}, w\right)\right\}\end{array}$ \\
\hline & $\begin{array}{l}S^{3} \times S^{3} \times S^{1} \\
\quad \supset\left\{\left(z^{p} w^{\lambda m_{-}}, z^{q} w^{\mu m_{-}}, w^{n_{-}}\right)\right\} H,\left\{\left(z^{p} w^{\lambda m_{+}}, z^{q} w^{\mu m_{+}}, w^{n_{+}}\right)\right\} H \supset H \\
\text { where } H=H_{-} \cdot H_{+}, H_{0}=\left\{\left(z^{p}, z^{q}, 1\right)\right\}, K^{-} \neq K^{+}, p \mu-q \lambda=1 \\
\operatorname{gcd}\left(n_{-}, n_{+}, d\right)=1 \text {, where } d \text { is the index of } H \cap K_{0}^{-} \cap K_{0}^{+} \text {in } K_{0}^{-} \cap K_{0}^{+}\end{array}$ \\
\hline$V_{F}^{7}$ & $\begin{array}{r}S^{3} \times S^{3} \times S^{1} \supset\left\{\left(e^{i p \phi} e^{i a \theta}, e^{i \phi}, e^{i \theta}\right)\right\}, S^{3} \times S^{1} \times \mathbb{Z}_{n} \supset\left\{\left(e^{i p \phi}, e^{i \phi}, 1\right)\right\} \cdot \hat{H} \\
\mathbb{Z}_{n} \simeq \hat{H} \subset\left\{\left(e^{i a \theta}, 1, e^{i \theta}\right)\right\}\end{array}$ \\
\hline$N_{G}$ & $\mathrm{U}(3) \supset \mathrm{S}(\mathrm{U}(1) \mathrm{U}(2)), \mathrm{S}(\mathrm{U}(1) \mathrm{U}(2)) \supset T^{2}$ \\
\hline$N_{H}^{7}$ & $\begin{array}{c}\mathrm{SU}(3) \times S^{1} \supset\left\{\left(\beta\left(m_{-} \theta\right), e^{i n_{-} \theta}\right)\right\} \cdot H,\left\{\left(\beta\left(m_{+} \theta\right), e^{i n_{+} \theta}\right)\right\} \cdot H \supset H \\
H_{0}=\mathrm{SU}(1) \mathrm{SU}(2) \times 1, \quad H=H_{-} \cdot H_{+}, \quad K^{-} \neq K^{+}, \\
\beta(\theta)=\operatorname{diag}\left(e^{-i \theta}, e^{i \theta}, 1\right), \quad \operatorname{gcd}\left(n_{-}, n_{+}, d\right)=1 \\
\text { where } d \text { is the index of } H \cap K_{0}^{-} \cap K_{0}^{+} \text {in } K_{0}^{-} \cap K_{0}^{+}\end{array}$ \\
\hline$N_{I}^{7}$ & $\mathrm{p}(2) \supset \mathrm{Sp}(1) \mathrm{Sp}(1), \mathrm{Sp}(1) \mathrm{Sp}(1) \supset \mathrm{Sp}(1) \mathrm{SO}(2)$ \\
\hline
\end{tabular}

Table II. The nonprimitive cohomogeneity one manifolds from Theorem A. 
In particular, the actions of type $P^{5}$ are all actions on $\mathrm{SU}(3) / \mathrm{SO}(3)$, and the actions of type $N^{5}$ are either on $S^{3} \times S^{2}$ or the nontrivial $S^{3}$ bundle over $S^{2}$, depending on the parameters.

For dimension 6, Hoelscher [2010a] found the analogous result by identifying the 6-manifolds that remain from Theorem A up to diffeomorphism. Such a result in dimension 7 would be much more difficult; however the first steps are taken in [Escher and Ultman 2008], where the cohomology rings of the 7-manifolds from Table I are computed, and in [Hoelscher 2010b], where the homology groups of the 7-manifolds from Table II are computed up to a group extension problem in a few cases.

The paper is organized as follows. In Section 1, we discuss cohomogeneity one manifolds in general and develop some basic facts that will be useful throughout. The classification will take place in Sections 2 to 4. Next, in Sections 5 through 7 we look at some of the actions in more detail and prove the main theorems.

\section{Cohomogeneity one manifolds}

In this section we will discuss the cohomogeneity one action of a Lie group $G$ on a manifold $M$ in general. We first review the basic structure of such actions, and see that they are completely determined by certain isotropy subgroups. We will then discuss how we can determine the fundamental group of the manifold from these isotropy groups. We will also give some helpful restrictions on the possible groups that can occur in our situation.

Throughout this section, $G$ will denote a compact connected Lie group and $M$ will be a closed and connected manifold, unless explicitly stated otherwise.

1.1. Basic structure. The action of a compact Lie group $G$ on a manifold $M$ is said to be cohomogeneity one if there are orbits of codimension 1, or equivalently if the orbit space $M / G$ is one-dimensional. If $M$ is connected it follows that $M / G$ is either $(-\infty, \infty),[0, \infty),[-1,1]$ or $S^{1}$. In the first two cases, $M$ will not be compact and in the last case $M$ will not be simply connected, since it will be fibered over a circle. We are only interested in compact simply connected manifolds so we will henceforth restrict our attention to those $M$ with $M / G \approx[-1,1]$. We will refer to such manifolds as interval cohomogeneity one manifolds.

To review the well-known structure of $M$ further, choose an arbitrary but fixed $G$-invariant Riemannian metric on $M$, and let $\pi: M \rightarrow M / G \approx[-1,1]$ denote the projection. Let $c:[-1,1] \rightarrow M$ be a minimal geodesic between the two nonprincipal orbits $\pi^{-1}(-1)$ and $\pi^{-1}(1)$. It then follows that $c$ meets all orbits orthogonally and that the isotropy group of $G$ is constant on the interior of $c$. For convenience, reparameterize the quotient interval $M / G \approx[-1,1]$ so that $\pi \circ$ $c=\mathrm{id}_{[-1,1]}$. Denote the isotropy groups by $H=G_{c(0)}=G_{c(t)}$ for $t \in(-1,1)$ and 
$K^{ \pm}=G_{c( \pm 1)}$ and let $D_{ \pm}$denote the disk of radius 1 normal to the nonprincipal orbits $\pi^{-1}( \pm 1)=G \cdot c( \pm 1)$ at $c( \pm 1)$. One can see then that $K^{ \pm}$acts on $D_{ \pm}$and does so transitively on $\partial D_{ \pm}$with isotropy $H$ at $c(0)$. Therefore $S^{l_{ \pm}}:=\partial D_{ \pm}=$ $K^{ \pm} \cdot c(0) \approx K^{ \pm} / H$. The slice theorem [Bredon 1972] tells us that the tubular neighborhoods of the nonprincipal orbits have the form $\pi^{-1}[-1,0] \approx G \times{ }_{K^{-}} D_{-}$ and $\pi^{-1}[0,1] \approx G \times{ }_{K^{+}} D_{+}$. Therefore we have decomposed our manifold into two disk bundles $G \times{ }_{K^{ \pm}} D_{ \pm}$glued along their common boundary $\pi^{-1}(0)=G \cdot c(0) \approx$ $G / H$. That is,

$$
M \approx G \times_{K^{-}} D_{-} \cup_{G / H} G \times_{K^{+}} D_{+}, \quad \text { where } S^{l_{ \pm}}=\partial D_{ \pm} \approx K^{ \pm} / H .
$$

This describes $M$ entirely in terms of $G$ and the isotropy groups $K^{ \pm} \supset H$. The collection of $G$ with its isotropy groups $G \supset K^{+}, K^{-} \supset H$ is called the group diagram of the cohomogeneity one manifold. Note: In the group diagram we understand that $G$ contains both subgroups $K^{-}$and $K^{+}$and that both $\mathrm{K}^{-}$and $\mathrm{K}^{+}$ contain $H$ as a subgroup.

Conversely, let $G \supset K^{+}, K^{-} \supset H$ be compact groups with $K^{ \pm} / H \approx S^{l_{ \pm}}$. We know from the classification of transitive actions on spheres [Besse 1978, page 195] that the $K^{ \pm}$action on $S^{l_{ \pm}}$must be linear and hence it extends to an action on the disk $D_{ \pm}$bounded by $S^{l_{ \pm}}$for each \pm . Therefore one can construct a cohomogeneity one manifold $M$ using (1-1). So a cohomogeneity one manifold $M$ with $M / G \approx[-1,1]$ determines a group diagram $G \supset K^{+}, K^{-} \supset H$ with $K^{ \pm} / H \approx S^{l_{ \pm}}$; conversely, such a group diagram determines a cohomogeneity one action. This reduces classifying such cohomogeneity one manifolds to finding subgroups of compact groups with certain properties.

Recall an action of $G$ on $M$ is effective if no element $g \in G$ fixes $M$ pointwise, except $g=1$. We claim that a cohomogeneity one action, as above, is effective if and only if $G$ and $H$ do not share any nontrivial normal subgroups. It is clear that if $N$ is the ineffective kernel of the $G$ action, that is, $N=\operatorname{ker}(G \rightarrow \operatorname{Diff}(M))$, then $N$ will be a normal subgroup of both $G$ and $H$. Conversely, let $N$ be the largest normal subgroup shared by $G$ and $H$. Then, as above, $N$ fixes the entire geodesic $c$ pointwise. Therefore, since $N$ is normal, it fixes all of $M$ pointwise. So it is easy to determine the effective version of any cohomogeneity one action from its group diagram alone. Because of this, we will generally allow our actions to be ineffective; however we will be most interested in the almost effective actions, that is, actions with at most a finite ineffective kernel. In this case, $N$ is a discrete normal subgroup and hence $N \subset Z(G)$, where $Z(G)$ is the center of $G$. Then in fact $N=H \cap Z(G)$ in this case, by what we said above.

The question of whether or not two different group diagrams determine the same action will be important to understand. We say the action of $G_{1}$ on $M_{1}$ is strictly equivalent to the action of $G_{2}$ on $M_{2}$ if there is a diffeomorphism $f: M_{1} \rightarrow M_{2}$ and 
an isomorphism $\phi: G_{1} \rightarrow G_{2}$ such that $f(g \cdot x)=\phi(g) \cdot f(x)$ for all $x \in M_{1}$ and $g \in G_{1}$. Similarly we say the actions of $G_{1}$ and $G_{2}$ on $M_{1}$ and $M_{2}$, respectively, are (effectively) equivalent if their effective versions are strictly equivalent. We will classify cohomogeneity one actions up to this type of equivalence. However, when $G_{1}=G_{2}$, a stronger type of equivalence is sometimes preferred: A map $f: M_{1} \rightarrow M_{2}$ between $G$-manifolds is $G$-equivariant if $f(g \cdot x)=g \cdot f(x)$ for all $x \in M_{1}$ and $g \in G$. The next proposition, taken from [Grove et al. 2008], applies to $G$-equivariant diffeomorphisms.

Proposition 1.2. Let a cohomogeneity one action of $G$ on $M$ be given by the group diagram $G \supset K^{-}, K^{+} \supset H$. Then any of the following operations on the group diagram will result in a $G$-equivariantly diffeomorphic manifold.

(i) Switching $K^{-}$and $K^{+}$.

(ii) Conjugating each group in the diagram by the same element of $G$.

(iii) Replacing $K^{-}$with $a K^{-} a^{-1}$ for $a \in N(H)_{0}$.

Conversely, the group diagrams for two G-equivariantly diffeomorphic manifolds must be taken to each other by some combination of these three operations.

The next corollary is particularly helpful when finding the group diagram for a given cohomogeneity one action. Recall that the groups $\mathrm{K}^{-}, \mathrm{K}^{+}$and $H$ are the isotropy groups along a minimal geodesic between nonprincipal orbits, with respect to some $G$ invariant metric on $M$. In most cases it is not convenient to explicitly find such a metric and geodesic. The following corollary solves this problem.

Corollary 1.3. Let $M$ be an interval cohomogeneity one manifold for the group $G$ and let $\gamma:[-1,1] \rightarrow M$ be any continuous curve between nonprincipal orbits that meets each orbit precisely once and that is differentiable at the nonprincipal orbits with derivative transverse to these orbits. If $\gamma$ satisfies the further property $G_{\gamma(t)}=G_{\gamma(0)}$ for all $t \in(-1,1)$, then $G \supset G_{\gamma(-1)}, G_{\gamma(1)} \supset G_{\gamma(0)}$ is a valid group diagram for the action of $G$ on $M$.

Proof. Fix a $G$ invariant metric on $M$, and let $c:[-1,1] \rightarrow M$ be a minimal geodesic between nonprincipal orbits. Then the group diagram along $c$ is given by $K^{ \pm}=G_{c( \pm 1)}$ and $H=G_{c(0)}$. After reparameterizing $\gamma$ we can assume that $\gamma(t)$ and $c(t)$ are in the same $G$-orbit for each $t \in[-1,1]$. This reparametrization will not affect the property of the derivative of $\gamma$ at the nonprincipal orbits. After applying some element of $G$ to $c$, we can also assume that $c(0)=\gamma(0)$.

Since the principal part of $M$ is $G$ equivariantly diffeomorphic to $G / H \times(-1,1)$, we can write $\gamma(t)=g(t) c(t)$ for some continuous curve $g:(-1,1) \rightarrow G$, with $g(0)=e$. That $\gamma^{\prime}( \pm 1)$ exists and is transverse to the nonprincipal orbits means that we can extend $g(t)$ to a continuous function on $[-1,1]$ with $\gamma(t)=g(t) c(t)$. 
We have $G_{\gamma(t)}=G_{g(t) c(t)}=g(t) G_{c(t)} g(t)^{-1}$ for all $t$. Since $\gamma(0)=c(0)$ and by our hypothesis, we also know $G_{\gamma(t)}=G_{\gamma(0)}=H$ for all $t \in(-1,1)$. Therefore $H=G_{\gamma(t)}=g(t) G_{c(t)} g(t)^{-1}=g(t) H g(t)^{-1}$ for all $t \in(-1,1)$. Since $g(0)=e$ it follows that $g(t) \in N(H)_{0}$ for all $t \in(-1,1)$. By continuity it follows that $n_{ \pm}:=g( \pm 1) \in N(H)_{0}$ as well. Putting this together we find that the diagram $G \supset G_{\gamma(-1)}, G_{\gamma(1)} \supset G_{\gamma(0)}$ is the diagram $G \supset n_{-} K^{-} n_{-}^{-1}, n_{+} K^{+} n_{+}^{-1} \supset H$, which represents our original action by Proposition 1.2.

Definition 1.4. We say the cohomogeneity one manifold $M_{G}$ is nonprimitive if it has some group diagram representation $G \supset K^{-}, K^{+} \supset H$ for which there is a proper connected closed subgroup $L \subset G$ with $L \supset K^{-}, K^{+}$. It then follows that $L \supset K^{-}, K^{+} \supset H$ is a group diagram that determines some cohomogeneity one manifold $M_{L}$.

Example. As an example, consider the group diagram $S^{3} \supset\left\{e^{i \theta}\right\},\left\{e^{j \theta}\right\} \supset\{ \pm 1\}$. There is no proper subgroup $L$ that contains both $K^{-}=\left\{e^{i \theta}\right\}$ and $K^{+}=\left\{e^{j \theta}\right\}$. However, by Proposition 1.2 this action is equivalent to the action with group diagram $S^{3} \supset\left\{e^{i \theta}\right\},\left\{e^{i \theta}\right\} \supset\{ \pm 1\}$. So in fact, this action is primitive.

Proposition 1.5. Take a nonprimitive cohomogeneity one manifold $M_{G}$ with $L$ and $M_{L}$ as in Definition 1.4. Then $M_{G}$ is $G$-equivariantly diffeomorphic to $G \times{ }_{L} M_{L}=$ $\left(G \times M_{L}\right) / L$, where L acts on $G \times M_{L}$ by $\ell \star(g, x)=\left(g \ell^{-1}, \ell x\right)$. Hence there is a fiber bundle $M_{L} \rightarrow M_{G} \rightarrow G / L$.

Proof. Let $c$ be a minimal geodesic in $M_{L}$ between nonprincipal orbits. Then it is clear that the curve $\tilde{c}(t)=(1, c(t)) \in G \times M_{L}$ is a geodesic where we equip $G \times M_{L}$ with the product metric for the biinvariant metric on $G$. It is also clear that $\tilde{c}$ is perpendicular to the $L$ orbits in $G \times M_{L}$. Therefore $c$ descends to a geodesic $\hat{c}$ in $G \times{ }_{L} M_{L}$, which is perpendicular to the $G$ orbits. The isotropy groups of the $G$ actions on $G \times{ }_{L} M_{L}$ are clearly given by $G_{\hat{c}(t)}=L_{c(t)}$, and hence this $G$ action on $G \times{ }_{L} M_{L}$ is cohomogeneity one with group diagram $G \supset K^{-}, K^{+} \supset H$.

1.6. The fundamental group. We will generally be looking at cohomogeneity one actions in terms of their group diagrams. Since this paper is concerned with simply connected cohomogeneity one manifolds, it will be important to be able to determine the fundamental group of the manifold using only the group diagram. In this section we will show how to do this and give strong but simple conditions on which group diagrams can give simply connected manifolds. Recall we are assuming that $G$ is compact and connected throughout this section.

Proposition 1.7 [Grove et al. 2008, Lemma 1.6]. Let $M$ be a compact simply connected cohomogeneity one manifold for the group $G$ as above. Then $M$ has no exceptional orbits, and hence, in the notation above, $l_{ \pm} \geq 1$, or equivalently $\operatorname{dim} K^{ \pm}>\operatorname{dim} H$. 
This next proposition can be considered as the van Kampen theorem for cohomogeneity one manifolds, and tells us precisely how to compute the fundamental groups from the group diagrams alone.

Proposition 1.8 (van Kampen). Let $M$ be the cohomogeneity one manifold given by the group diagram $G \supset K^{+}, K^{-} \supset H$ with $K^{ \pm} / H=S^{l_{ \pm}}$and assume $l_{ \pm} \geq 1$. Then $\pi_{1}(M) \approx \pi_{1}(G / H) / N_{-} N_{+}$, where

$$
N_{ \pm}=\operatorname{ker}\left\{\pi_{1}(G / H) \rightarrow \pi_{1}\left(G / K^{ \pm}\right)\right\}=\operatorname{Im}\left\{\pi_{1}\left(K^{ \pm} / H\right) \rightarrow \pi_{1}(G / H)\right\} .
$$

In particular $M$ is simply connected if and only if the images of $K^{ \pm} / H=S^{l_{ \pm}}$ generate $\pi_{1}(G / H)$ under the natural inclusions.

Proof. We compute the fundamental group of $M$ using van Kampen's theorem. In the notation of Section 1.1, we can decompose $M$ as $\pi^{-1}([-1,0]) \cup \pi^{-1}([0,1])$, where $\pi^{-1}([-1,0]) \cap \pi^{-1}([0,1])=G \cdot x_{0} \approx G / H$. We also know that, with a slight abuse of notation, $\pi^{-1}([0, \pm 1])$ deformation retracts to $\pi^{-1}( \pm 1)=G \cdot x_{ \pm} \approx G / K^{ \pm}$. So in fact we have the homotopy equivalence

$$
\pi^{-1}([0, \pm 1]) \rightarrow G / K^{ \pm}, \quad g \cdot c(t) \mapsto g K^{ \pm} .
$$

Therefore we may use the map induced by the projection $\pi_{1}(G / H) \rightarrow \pi_{1}\left(G / K^{ \pm}\right)$ in place of the map induced by the inclusion $\pi_{1}\left(G \cdot x_{0}, x_{0}\right) \rightarrow \pi_{1}\left(\pi^{-1}([0, \pm 1]), x_{0}\right)$, for van Kampen's theorem.

Now look at the fiber bundle

$$
K^{ \pm} / H \rightarrow G / H \rightarrow G / K^{ \pm}, \quad \text { where } K^{ \pm} / H \approx S^{l_{ \pm}} .
$$

This gives a long exact sequence of homotopy groups:

$$
\begin{array}{r}
\cdots \rightarrow \pi_{i}\left(S^{l_{ \pm}}\right) \stackrel{i_{*}^{ \pm}}{\longrightarrow} \pi_{i}(G / H) \stackrel{\rho_{*}^{ \pm}}{\longrightarrow} \pi_{i}\left(G / K^{ \pm}\right) \stackrel{\partial_{*}}{\longrightarrow} \pi_{i-1}\left(S^{l_{ \pm}}\right) \rightarrow \cdots \\
\cdots \rightarrow \pi_{1}\left(S^{l_{ \pm}}\right) \stackrel{i_{*}^{ \pm}}{\longrightarrow} \pi_{1}(G / H) \stackrel{\rho_{*}^{ \pm}}{\longrightarrow} \pi_{1}\left(G / K^{ \pm}\right) \stackrel{\partial_{*}}{\longrightarrow} \pi_{0}\left(S^{l_{ \pm}}\right)
\end{array}
$$

Notice that this implies $\rho_{*}^{ \pm}: \pi_{1}(G / H) \rightarrow \pi_{1}\left(G / K^{ \pm}\right)$is onto, since $l_{ \pm}>0$. In fact it follows $G / H \rightarrow G / K^{ \pm}$is $l_{ \pm}$-connected, but we will not need this.

By van Kampen's theorem, $\pi_{1}(M) \approx \pi_{1}(G / H) / N_{-} N_{+}$, where $N_{ \pm}=\operatorname{ker}\left(\rho_{*}^{ \pm}\right)$. Finally, by (1-3), we see $N_{ \pm}=\operatorname{ker}\left(\rho_{*}^{ \pm}\right)=\operatorname{Im}\left(i_{*}^{ \pm}\right)$, and this concludes the proof.

We now give a reformulation of [Grove et al. 2008, Lemma 1.6], which will be very convenient for dealing with the case that $l_{-}$or $l_{+}$is greater than 1 .

Corollary 1.9. Suppose $M$ is the cohomogeneity one manifold given by the group diagram $G \supset K^{+}, K^{-} \supset H$ with $K^{ \pm} / H=S^{l_{ \pm}}$.

(i) Suppose $l_{-} \geq 1$ and $l_{+}>1$ and hence $H \cap K_{0}^{+}=H_{0}$. Then $\pi_{1}(M) \approx \pi_{1}\left(G / K^{-}\right)$ and in particular, if $M$ is simply connected, $K^{-}$is connected. 
(ii) Suppose $l_{-}, l_{+}>1$. Then $\pi_{1}(M) \approx \pi_{1}(G / H) \approx \pi_{1}\left(G / K^{ \pm}\right)$and in particular, if $M$ is simply connected, all of $H, K^{-}$and $K^{+}$are connected.

This corollary tells us how to deal with the case that $l_{-}$or $l_{+}$is greater than one. For the case that both $l_{ \pm}=1$ the following lemma will be very helpful.

Lemma 1.10. Suppose $M$ is the cohomogeneity one manifold given by the group diagram $G \supset K^{+}, K^{-} \supset H$. Denote $H_{ \pm}=H \cap K_{0}^{ \pm}$and let $\alpha_{ \pm}:[0,1] \rightarrow K_{0}^{ \pm}$be curves that generate $\pi_{1}\left(K^{ \pm} / H\right)$, with $\alpha_{ \pm}(0)=1 \in G$. M is simply connected if and only if

(i) $H$ is generated as a subgroup by $H_{-}$and $H_{+}$, and

(ii) $\alpha_{-}$and $\alpha_{+}$generate $\pi_{1}\left(G / H_{0}\right)$.

Remark. The curves $\alpha_{ \pm}$are, in general, not loops in $G / H_{0}$. However we can compose them in $G / H_{0}$ either via pointwise multiplication in $G$ or via lifting their compositions in $G / H$, where they are loops, to $G / H_{0}$. When we say $\alpha_{ \pm}$generate $\pi_{1}\left(G / H_{0}\right)$, we mean the combinations of these curves that form loops in $G / H_{0}$ generate $\pi_{1}\left(G / H_{0}\right)$. Also notice that if $\operatorname{dim}\left(K^{-} / H\right)>1$, we can simply take $\alpha_{-}(t)=1$ for all $t$, and similarly for $\alpha_{+}$.

The fact that $\pi_{1}(M)=0$ implies (i) is equivalent to [Grove et al. 2008, Lemma 1.7]. Our independent proof here leads to the full version of this lemma.

Proof. By Proposition 1.8, $M$ is simply connected if and only if $\pi_{1}(G / H)=$ $\left\langle\alpha_{-}\right\rangle \cdot\left\langle\alpha_{+}\right\rangle$, where $\alpha_{ \pm}$are considered as loops in $G / H$. Furthermore, since $\alpha_{ \pm}$ are loops in $K^{ \pm} / H$, it follows that $a_{ \pm}:=\alpha_{ \pm}(1) \in K_{0}^{ \pm} \cap H=H_{ \pm}$. It is clear that the group generated by $H_{-}$and $H_{+}$is the same as the group generated by $a_{-}, a_{+}$ and $H_{0}$. Therefore condition (i) is equivalent to the statement that $H$ is generated by $a_{-}, a_{+}$and $H_{0}$.

First assume that $M$ is simply connected, so that $\pi_{1}(G / H)=\left\langle\alpha_{-}\right\rangle \cdot\left\langle\alpha_{+}\right\rangle$. Since the map $G / H_{0} \rightarrow G / H$ is a cover, it is clear that $\alpha_{-}$and $\alpha_{+}$generate $\pi_{1}\left(G / H_{0}\right)$. We must only show that $H$ is generated by $a_{-}, a_{+}$and $H_{0}$.

Choose an arbitrary component $h H_{0}$ of $H$. We claim that some product of $a_{-}$ and $a_{+}$will lie in $h H_{0}$. For this, let $\gamma:[0,1] \rightarrow G$ be an arbitrary path with $\gamma(0)=1$ and $\gamma(1) \in h H_{0}$. Then $\gamma$ represents a loop in $G / H$ and since $\pi_{1}(G / H)=$ $\left\langle\alpha_{-}\right\rangle \cdot\left\langle\alpha_{+}\right\rangle$, we must have $[\gamma]=\left[\alpha_{-}\right]^{n}\left[\alpha_{+}\right]^{m}$ for some $m, n \in \mathbb{Z}$, where $[\cdot]$ denotes the corresponding class in $\pi_{1}(G / H)$.

We now make use of the following observation. In general, for compact Lie groups $J \subset L$, take paths $\beta_{ \pm}:[0,1] \rightarrow L$, with $\beta_{ \pm}(0)=1$ and $\beta_{ \pm}(1) \in J$. Then we see that $\left(\beta_{-} \cdot \beta_{+}(1)\right) \circ \beta_{+}$is fixed endpoint homotopic to $\beta_{-} \cdot \beta_{+}$in $L$, where $\beta_{-} \cdot \beta_{+}(1)$ is the path $t \mapsto \beta_{-}(t) \cdot \beta_{+}(1)$, the symbol $\circ$ denotes path composition, and $\beta_{-} \cdot \beta_{+}$is the path $t \mapsto \beta_{-}(t) \cdot \beta_{+}(t)$. Therefore $\left[\beta_{-}\right]\left[\beta_{+}\right]=\left[\beta_{-} \cdot \beta_{+}\right]$as classes in $\pi_{1}(J / L)$. 
In our case, this implies $[\gamma]=\left[\alpha_{-}\right]^{n}\left[\alpha_{+}\right]^{m}=\left[\alpha_{-}^{n} \cdot \alpha_{+}^{m}\right]$ in $\pi_{1}(G / H)$. Now look at the cover $G / H_{0} \rightarrow G / H$. Since the paths $\gamma$ and $\alpha_{-}^{n} \cdot \alpha_{+}^{m}$ both start at $1 \in G$, it follows that $\gamma$ and $\alpha_{-}^{n} \cdot \alpha_{+}^{m}$ both end in the same component of $H$. Hence

$$
\left(\alpha_{-}^{n} \cdot \alpha_{+}^{m}\right)(1)=\alpha_{-}(1)^{n} \cdot \alpha_{+}(1)^{m}=a_{-}^{n} \cdot a_{+}^{m} \in h H_{0} .
$$

Therefore, $a_{-}, a_{+}$and $H_{0}$ generate $H$, proving (i).

Next suppose (i) and (ii) hold. Again, since the map $G / H_{0} \rightarrow G / H$ is a cover, $\pi_{1}(G / H)$ is generated by $\pi_{1}\left(G / H_{0}\right)$ and a collection of curves in $G / H_{0}$ that go from $H_{0}$ to each component of $H$. The curves $\alpha_{-}$and $\alpha_{+}$already generate $\pi_{1}\left(G / H_{0}\right)$ by assumption. Saying $a_{-}, a_{+}$and $H_{0}$ generate $H$ is equivalent to saying that combinations of $\alpha_{-}$and $\alpha_{+}$can reach any component of $H$, when considered as paths in $G / H_{0}$. Hence $\alpha_{ \pm}$generate $\pi_{1}(G / H)$, and $M$ is simply connected by Proposition 1.8.

1.11. Extensions and reductions. In this section we will describe a natural way of reducing certain cohomogeneity one actions to actions by smaller groups with the same orbits. We will also describe a way of extending actions to larger groups, and we will see that these two processes are inverses of each other.

Proposition 1.12. Let $M$ be the cohomogeneity one manifold given by the group diagram $G \supset K^{+}, K^{-} \supset H$ and suppose $G=G_{1} \times G_{2}$ with $\operatorname{proj}_{2}(H)=G_{2}$. Then the subaction of $G_{1} \times 1$ on $M$ is also by cohomogeneity one, with the same orbits, and with isotropy groups $K_{1}^{ \pm}=K^{ \pm} \cap\left(G_{1} \times 1\right)$ and $H_{1}=H \cap\left(G_{1} \times 1\right)$.

Proof. Recall that the action of $G$ on each orbit $G \cdot x$ is equivalent to the $G$ action on $G / G_{x}$. So it is enough to test the claim on each type of orbit: $G / K^{+}, G / K^{-}$ and $G / H$. Let $G / G_{x}$ be one such orbit and notice that $H \subset G_{x}$. Then for each element $\left(g_{1}, g_{2}\right) G_{x} \in G / G_{x}$, there is some element of $H$ of the form $\left(h_{1}, g_{2}\right)$ since $\operatorname{proj}_{2}(H)=G_{2}$. Then $\left(g_{1}, g_{2}\right) G_{x}=\left(g_{1} h_{1}^{-1}, 1\right) \cdot\left(h_{1}, g_{2}\right) G_{x}=\left(g_{1} h_{1}^{-1}, 1\right) G_{x}$ and hence an arbitrary point $\left(g_{1}, g_{2}\right) G_{x}$ is in the $G_{1} \times 1$ orbit of $(1,1) G_{x}$. This proves $G_{1} \times 1$ acts on $M$ with the same orbits as $G$ and hence still acts by cohomogeneity one. The fact that the isotropy groups of the $G_{1} \times 1$ action are $K_{1}^{ \pm}=K^{ \pm} \cap\left(G_{1} \times 1\right)$ and $H_{1}=H \cap\left(G_{1} \times 1\right)$ is then clear.

We will now describe a way of extending a given cohomogeneity one action to an action by a possibly larger group. Let $M$ be a cohomogeneity one manifold with group diagram $G_{1} \supset K_{1}^{-}, K_{1}^{+} \supset H_{1}$, and let $L$ be a compact connected subgroup of $N\left(H_{1}\right) \cap N\left(K_{1}^{-}\right) \cap N\left(K_{1}^{+}\right)$. Notice that $L \cap H_{1}$ is normal in $L$ and define $G_{2}:=L /\left(L \cap H_{1}\right)$. We then define an action by $G_{1} \times G_{2}$ on $M$ orbitwise by $\left(\hat{g}_{1},[l]\right) \star g_{1}\left(G_{1}\right)_{x}=\hat{g}_{1} g_{1} l^{-1}\left(G_{1}\right)_{x}$ on each orbit $G_{1} /\left(G_{1}\right)_{x}$ for $\left(G_{1}\right)_{x}=H_{1}$ or $K_{1}^{ \pm}$.

Definition 1.13. Such an extension is called a normal extension. 
Proposition 1.14. This extension describes a smooth action of $G:=G_{1} \times G_{2}$ on $M$ with the same orbits as $G_{1}$ and with group diagram

$$
G_{1} \times G_{2} \supset\left(K_{1}^{-} \times 1\right) \cdot \Delta L,\left(K_{1}^{+} \times 1\right) \cdot \Delta L \supset\left(H_{1} \times 1\right) \cdot \Delta L,
$$

where $\Delta L=\{(l,[l]) \mid l \in L\}$.

Proof. Clearly this action is well defined and has the same orbits as the original $G_{1}$ action. Now let $c:[-1,1] \rightarrow M$ be a minimal geodesic between nonprincipal orbits in $M$ such that $\left(G_{1}\right)_{c(t)}=H_{1}$ for $t \in(-1,1)$ and $\left(G_{1}\right)_{c( \pm 1)}=K_{1}^{ \pm}$. Then it is clear that the isotropy subgroups of $G=G_{1} \times G_{2}$ are

$$
H:=G_{c(t)}=H_{1} \cdot \Delta L \quad \text { for } t \in(-1,1) \text { and } K^{ \pm}:=G_{c( \pm 1)}=K_{1}^{ \pm} \cdot \Delta L,
$$

where we are identifying $G_{1}$ with $G_{1} \times 1$. So if we can show that the action is smooth and that there is a $G$-invariant metric on $M$ such that $c$ is a minimal geodesic, we will be done.

Let $\bar{M}$ be the manifold with group diagram $G \supset K^{-}, K^{+} \supset H$, with the corresponding geodesic $\bar{c}$, as above. Note that $\operatorname{proj}_{2}(H)=\operatorname{proj}_{2}\left(H_{1} \cdot \Delta L\right)=G_{2}$. Hence by Proposition $1.12, G_{1}$ still acts isometrically on $\bar{M}$ by cohomogeneity one with isotropy groups

$$
\left(H_{1} \cdot \Delta L\right) \cap\left(G_{1} \times 1\right)=H_{1} \times 1 \quad \text { and } \quad\left(K_{1}^{ \pm} \cdot \Delta L\right) \cap\left(G_{1} \times 1\right)=K_{1}^{ \pm} \times 1 .
$$

So $\bar{M}$ and $M$ are $G_{1}$-equivariantly diffeomorphic, by the map $\phi: g_{1} \cdot \bar{c}(t) \mapsto g_{1} \cdot c(t)$.

We now claim that $\phi$ is also $G$-equivariant. To see this define the set-theoretic map $\psi: \bar{M} \rightarrow M, g \cdot \bar{c}(t) \mapsto g \cdot c(t)$. This is well defined since $G$ has the same isotropy group at $\bar{c}(t)$ as at $c(t)$. This set map is clearly $G$-equivariant, by definition. By restricting to elements of the form $g_{1} \cdot \bar{c}(t)$ for $g_{1} \in G_{1}$, we see that $\psi\left(g_{1} \cdot \bar{c}(t)\right)=\phi\left(g_{1} \cdot \bar{c}(t)\right)$. Since the $G_{1}$ orbits are equal to the $G$ orbits in $\bar{M}$ by Proposition 1.12, $\psi=\phi$ as maps. In particular, $\psi$ is a diffeomorphism since $\phi$ is. Therefore $\bar{M}$ is $G$-equivariantly diffeomorphic to $M$.

Proposition 1.15. For $M$ as in Proposition 1.12, the action by $G=G_{1} \times G_{2}$ occurs as a normal extension of the reduced action of $G_{1} \times 1$ on $M$.

Proof. We first claim that we can assume $H \cap\left(1 \times G_{2}\right)=1$, which will be useful for technical reasons. To see this, suppose $H_{2}:=H \cap\left(1 \times G_{2}\right)$ is nontrivial. $H_{2}$ is obviously normal in $H$, and it is also normal in $G$ since $\operatorname{proj}_{2}(H)=G_{2}$. Then there is a more effective version of the same action by

$$
\left(G_{1} \times G_{2}\right) / H_{2} \approx G_{1} \times\left(G_{2} / H_{2}\right)=: G_{1} \times \tilde{G}_{2} .
$$

We still have $\operatorname{proj}_{2}(\tilde{H})=\tilde{G}_{2}$ for this action, where $\tilde{H}$ is the new principal isotropy group, and this time $\tilde{H} \cap\left(1 \times \tilde{G}_{2}\right)=1$. So assume $H \cap\left(1 \times G_{2}\right)=1$. 
Consider the reduced action with diagram $G_{1} \times 1 \supset K_{1}^{-} \times 1, K_{1}^{+} \times 1 \supset H_{1} \times 1$ from Proposition 1.12. Let $L=\operatorname{proj}_{1}\left(H_{0}\right) \subset G_{1}$. We claim that the original $G_{1} \times G_{2}$ action is equivalent to the normal extension of the $G_{1}$ action via $L$. First notice that since $H_{1}$ is normal in $H$, it is also normal in $L=\operatorname{proj}_{1}\left(H_{0}\right)$. Similarly $L$ is in the normalizer of $K_{1}^{ \pm}$. So in fact $L \subset N\left(H_{1}\right) \cap N\left(K_{1}^{-}\right) \cap N\left(K_{1}^{+}\right)$.

Now, notice the map $\operatorname{proj}_{1}: H_{0} \rightarrow L=\operatorname{proj}_{1}\left(H_{0}\right)$ is onto with trivial kernel, since we assumed $H \cap\left(1 \times G_{2}\right)=1$. Therefore $\operatorname{proj}_{1}$ is a Lie group isomorphism and hence has an inverse $\psi: L \rightarrow H_{0}$ which must have the form $\psi(l)=(l, \phi(l))$ for some map $\phi: L \rightarrow G_{2}$. Notice that $\phi$ maps $L$ onto $G_{2}$ with kernel $H_{1} \cap L$. Therefore $G_{2} \approx L /\left(H_{1} \cap L\right)$, via $\phi$.

Notice that $H_{0}=\psi(L)=\{(l, \phi(l))\}$. It is also clear that $H=H_{1} \cdot H_{0}$ and similarly $K^{ \pm}=K_{1}^{ \pm} \cdot H_{0}$. Therefore we can write the group diagram for our original $G_{1} \times G_{2}$ action as

$$
G_{1} \times G_{2} \supset K_{1}^{-} \cdot H_{0}, K_{1}^{+} \cdot H_{0} \supset H_{1} \cdot H_{0} .
$$

Then, after the isomorphism $G_{1} \times G_{2} \rightarrow G_{1} \times\left(L /\left(H_{1} \cap L\right)\right),\left(g_{1}, \phi(l)\right) \mapsto\left(g_{1},[l]\right)$, $H_{0}$ becomes $\Delta L:=\{(l,[l])\}$ and this diagram becomes exactly the diagram in (1-4). Therefore the original action by $G_{1} \times G_{2}$ is equivalent to the normal extension of the $G_{1}$ action along $L$.

Definition 1.16. We say the cohomogeneity one action of a group $G$ on a manifold $M$ is reducible if there is a proper closed normal subgroup of $G$ that acts on $M$ with the same orbits.

Every compact connected Lie group has a cover of the form $G_{1} \times \cdots \times G_{l} \times T^{n}$, where the $G_{i}$ are simple Lie groups. Therefore every cohomogeneity one action can be given almost effectively with $G=G_{1} \times \cdots \times G_{l} \times T^{n}$. In this case, we claim the action is reducible if and only if $H$ projects onto some factor of $G$. Proposition 1.12 proves this claim in one direction. Conversely, suppose that some proper closed normal subgroup $N$ of $G$ acts by cohomogeneity one with the same orbits. Since the orbits of $G$ are connected, we can assume that $N$ is connected. Therefore $N=\prod_{i \in I} G_{i} \times T^{p}$ for some subset $I \subset\{1, \ldots, l\}$ and some $T^{p} \subset T^{n}$. Then let $L=\prod_{i \notin I} G_{i} \times T^{q}$, where $T^{p} \times T^{q}=T^{n}$, so that $G=N \times L$. The assumption that $N$ acts on $M$ with the same orbits means that $N$ acts transitively on $G / H=(N \times L) / H$. This means we can write any element $(n, \ell) H \in G / H$ as $(\tilde{n}, 1) H$, and hence $H$ must project onto $L$.

Most importantly, this section shows that the classification of cohomogeneity one manifolds is quickly reduced to the classification of the nonreducible ones. Therefore we will assume in our classification that all our actions are nonreducible and we will loose little generality, since every other cohomogeneity one action will be a normal extension of a nonreducible action. 
1.17. More limitations on the groups. This section gives a few more restrictions on the groups that can act by cohomogeneity one on simply connected manifolds. The first addresses the case that the group has an abelian factor.

Proposition 1.18. Let $M$ be the cohomogeneity one manifold given by the group diagram $G \supset K^{+}, K^{-} \supset H$, where $G=G_{1} \times T^{m}$ acts almost effectively and nonreducibly and $G_{1}$ is semisimple. Then we know $H_{0}=H_{1} \times 1 \subset G_{1} \times 1$. Further, if $M$ is simply connected, then $m \leq 2$ and we have the following:

(i) If $m=1$, then at least one of $\operatorname{proj}_{2}\left(K_{0}^{ \pm}\right)$is equal to $S^{1}$, say $\operatorname{proj}_{2}\left(K_{0}^{-}\right)$. Then $K^{-} / H \approx S^{1}$ and $K^{-}=S_{-}^{1} \cdot H$ for a circle group $S_{-}^{1}$, with $\operatorname{proj}_{2}\left(S_{-}^{1}\right)=S^{1}$. Furthermore, if $\operatorname{rank}(H)=\operatorname{rank}\left(G_{1}\right)$ or if $H_{1}$ is maximal-connected in $G_{1}$, then $H, K^{-}$and $K^{+}$are all connected, $K^{-}=H_{1} \times S^{1}$, and $K^{+}$is either $H_{1} \times S^{1}$ or has the form $K_{1} \times 1$ for $K_{1} / H_{1} \approx S^{l_{+}}$.

(ii) If $m=2$, then $K^{ \pm} / H$ are both circles and $K^{ \pm}=S_{ \pm}^{1} \cdot H$ for circle groups $S_{ \pm}^{1}$, with $\operatorname{proj}_{2}\left(S_{-}^{1}\right) \cdot \operatorname{proj}_{2}\left(S_{+}^{1}\right)=T^{2}$. Furthermore, if $\operatorname{rank}(H)=\operatorname{rank}\left(G_{1}\right)$, then the $G$ action is equivalent to the product action of $G_{1} \times T^{2}$ on $\left(G_{1} / H_{1}\right) \times S^{3}$, where $T^{2}$ acts on $S^{3} \subset \mathbb{C}^{2}$ by component-wise multiplication.

Proof. In all cases $\operatorname{proj}_{2}\left(K_{0}^{ \pm}\right)$is a compact connected subgroup of $T^{m}$. Now say $\operatorname{proj}_{2}\left(K_{0}^{-}\right)$is nontrivial. It must then be a torus $T^{n} \subset T^{m}$. Then we have $\operatorname{proj}_{2}: K_{0}^{-} \rightarrow T^{n}$ with kernel $K_{0}^{-} \cap\left(G_{1} \times 1\right)$. Therefore we have the fiber bundle

$$
\left(K_{0}^{-} \cap\left(G_{1} \times 1\right)\right) /\left(H_{1} \times 1\right) \rightarrow K_{0}^{-} /\left(H_{1} \times 1\right) \rightarrow K_{0}^{-} /\left(K_{0}^{-} \cap\left(G_{1} \times 1\right)\right) \approx T^{n},
$$

which gives a piece of a long exact sequence:

$$
\pi_{1}\left(K_{0}^{-} /\left(H_{1} \times 1\right)\right) \rightarrow \pi_{1}\left(T^{n}\right) \rightarrow \pi_{0}\left(\left(K_{0}^{-} \cap\left(G_{1} \times 1\right)\right) /\left(H_{1} \times 1\right)\right) .
$$

The last group in this sequence is finite and the middle group is infinite. This means that $K_{0}^{-} / H_{0}$ has infinite fundamental group. Given that this space is a sphere, it follows that $K^{-} / H \approx S^{1}$. Therefore $K_{0}^{-}=H_{0} \cdot S_{-}^{1}$ for some circle group $S_{-}^{1}$ with $\operatorname{proj}_{2}\left(S_{-}^{1}\right)=S^{1} \subset T^{m}$. Similarly, if $\operatorname{proj}_{2}\left(K_{0}^{+}\right)$is nontrivial, then $K_{0}^{+}=H_{0} \cdot S_{+}^{1}$ for $S_{+}^{1}$ with $\operatorname{proj}_{2}\left(S_{+}^{1}\right)=S^{1} \subset T^{m}$.

We know that $\operatorname{proj}_{2}\left(K_{0}^{-}\right)$and $\operatorname{proj}_{2}\left(K_{0}^{+}\right)$generate some torus $T^{n}$ in $T^{m}$, with $n \leq 2$. It is clear that if $m>n$ then $K^{-} / H$ and $K^{+} / H$ will not generate $\pi_{1}(G / H)$ and hence $M$ will not be simply connected, by Proposition 1.8. Therefore, $m \leq 2$, and if $m=1$ then one of $K^{ \pm}$must be a circle as above, and if $m=2$ then both $K^{ \pm}$ must be circles as above. This proves the first part of the proposition.

For the second part, if rank $H_{1}=\operatorname{rank} G_{1}$ or if $H_{1}$ is maximal-connected in $G_{1}$, we first claim that $\operatorname{proj}_{1}\left(K_{0}^{-}\right)=H_{1}$ if $K^{-} / H \approx S^{1}$. In the case that $H_{1}$ is maximal in $G_{1}$ this is clear since if $\operatorname{proj}_{1}\left(K_{0}^{-}\right)$is larger than $H_{1}$ it would be all of $G_{1}$. Yet there is no compact semisimple group $G_{1}$ with subgroup $H_{1}$ where $G_{1} / H_{1} \approx S^{1}$. For the case that $\operatorname{rank}\left(H_{1}\right)=\operatorname{rank}\left(G_{1}\right)$, recall that for a general compact Lie group, the rank 
and the dimension have the same parity modulo 2 . Since $K^{-}=S_{-}^{1} \cdot H, \operatorname{proj}_{1}\left(K_{0}^{-}\right)$is at most one dimension larger than $H_{1}$. But if $\operatorname{proj}_{1}\left(K_{0}^{-}\right)$is of one higher dimension than $H_{1}$ it would follow that $\operatorname{rank}\left(\operatorname{proj}_{1}\left(K_{0}^{-}\right)\right)=\operatorname{rank}\left(H_{1}\right)+1=\operatorname{rank}\left(G_{1}\right)+1$, a contradiction since $\operatorname{proj}_{1}\left(K_{0}^{-}\right) \subset G_{1}$. Therefore $\operatorname{proj}_{1}\left(K_{0}^{-}\right)=H_{1}$ in either case. Then since $K^{-}=S_{-}^{1} \cdot H$, it follows that $K_{0}^{-}=H_{1} \times S_{-}^{1} \subset G_{1} \times T^{m}$. Similarly if $K^{+} / H \approx S^{1}$, then $K_{0}^{+}=H_{1} \times S_{+}^{1}$.

To see that all the groups are connected in this case, we note that if $K_{0}^{-} \cap H$ is not $H_{0}$, then $H \cap 1 \times S^{1}$ is nontrivial and there is a more effective action for the same groups with $H \cap 1 \times S^{1}=1$. So we can assume that $K_{0}^{-} \cap H=H_{0}$. If in addition $K^{+} / H \approx S^{1}$, then by the same argument $K_{0}^{+} \cap H=H_{0}$ as well. If $\operatorname{dim}\left(K^{+} / H\right)>1$ then $K_{0}^{+} \cap H=H_{0}$ already, since $K_{0}^{+} /\left(H \cap K_{0}^{+}\right)$would be a simply connected sphere. In any case we know that $K_{0}^{ \pm} \cap H=H_{0}$. Then, by Lemma 1.10, $H$ must be connected, forcing $K^{-}$and $K^{+}$to be connected as well.

Now, it only remains to prove the last statement of (ii). In this case we already know $K^{-}=H_{1} \times S_{-}^{1}$ and $K^{+}=H_{1} \times S_{+}^{1}$. It is then clear that $K^{-} / H$ and $K^{+} / H$ generate $\pi_{1}(G / H) \approx \pi_{1}\left(\left(G_{1} / H_{1}\right) \times T^{2}\right)$ if and only if $S_{-}^{1}$ and $S_{+}^{1}$ generate $\pi_{1}\left(T^{2}\right)$. This happens precisely when there is an automorphism of $T^{2}$ taking $S_{-}^{1}$ to $S^{1} \times 1$ and $S_{+}^{1}$ to $1 \times S^{1}$. From Proposition 1.8 we can assume this automorphism exists. After this automorphism the group diagram has the form

$$
G_{1} \times S^{1} \times S^{1} \supset H_{1} \times S^{1} \times 1, H_{1} \times 1 \times S^{1} \supset H_{1} \times 1 \times 1 .
$$

It is easy to check that this action is the action described in the proposition (see Section 1.21 for more details).

The next two propositions give the possible dimensions that the group $G$ can have, if it acts by cohomogeneity one.

Proposition 1.19. If a Lie group $G$ acts almost effectively and by cohomogeneity one on the manifold $M^{n}$, then $n-1 \leq \operatorname{dim}(G) \leq n(n-1) / 2$.

Proof. Recall that $\operatorname{dim} G / H=n-1$ for a principal orbit $G \cdot x \approx G / H$, so the first inequality is trivial. Now we claim that $G$ also acts almost effectively on a principal orbit $G \cdot x \approx G / H$. So suppose an element $g \in G$ fixes $G \cdot x$ pointwise. Then in particular $g \in H$. We saw above that $H$ fixes the geodesic $c$ pointwise and hence $g$ fixes all of $M$ pointwise. So, in fact, $G$ acts almost effectively on $G / H$. Now equip $G / H$ with a $G$ invariant metric. It then follows that $G$ maps into Isom $G / H$ with finite kernel. Since $\operatorname{dim} G / H=n-1$, we know $\operatorname{dim}(\operatorname{Isom} G / H) \leq n(n-1) / 2$ and this proves the second inequality.

The following treats the special case where $G$ has the largest possible dimension.

Proposition 1.20. Suppose $G$ is a compact Lie group that acts almost effectively and by cohomogeneity one on the manifold $M^{n}$ with $n>2$, with group diagram 
$G \supset K^{-}, K^{+} \supset H$ and no exceptional orbits. If $\operatorname{dim}(G)=n(n-1) / 2$ and $G$ is simply connected, then $G$ is isomorphic to $\operatorname{Spin}(n)$ and the action is equivalent to the $\operatorname{Spin}(n)$ action on $S^{n} \subset \mathbb{R}^{n} \times \mathbb{R}$, where $\operatorname{Spin}(n)$ acts on $\mathbb{R}^{n}$ via $\mathrm{SO}(n)$, leaving $\mathbb{R}$ pointwise fixed.

Proof. First, since $G$ acts on $M$ almost effectively, we know that $G$ also acts almost effectively on the principal orbits that are equivariantly diffeomorphic to $G / H$. Now endow $G / H$ with the metric induced from a biinvariant metric on $G$, so that $G$ acts by isometry. Therefore we have a Lie group homomorphism $G \rightarrow \operatorname{Isom} G / H$ with finite kernel. Since $\operatorname{dim} G=n(n-1) / 2$ and $\operatorname{dim} G / H=n-1$, it follows that $G / H$ must be a space form [Petersen 1998]. Further, since $G$ is simply connected, it follows that $G / H_{0}$ is a compact simply connected space form. Hence $G / H_{0}$ is isometric to $S^{n-1}$ and $G$ still acts almost effectively and by isometry on $S^{n-1}$. So in fact $G \rightarrow$ Isom $S^{n-1}=\mathrm{SO}(n)$ as a Lie group homomorphism with finite kernel. Since $\operatorname{dim} G=\operatorname{dim} \operatorname{SO}(n)$, it follows that $G$ is isomorphic to $\operatorname{Spin}(n)$. We also know that the only way $\operatorname{Spin}(n)$ can act transitively on an $(n-1)$-sphere is with $\operatorname{Spin}(n-1)$ isotropy; see [Besse 1978, page 195]. Therefore there is an isomorphism $G \rightarrow \operatorname{Spin}(n)$ taking $H_{0}$ to $\operatorname{Spin}(n-1)$.

We also see that $\operatorname{Spin}(n-1)$ is maximal among connected subgroups of $\operatorname{Spin}(n)$. Hence $K^{ \pm}$must both be $\operatorname{Spin}(n)$ and hence $H$ is connected since $n>2$. Therefore the group diagram for this action is $\operatorname{Spin}(n) \supset \operatorname{Spin}(n), \operatorname{Spin}(n) \supset \operatorname{Spin}(n-1)$. It is easy to check that the $\operatorname{Spin}(n)$ action on $S^{n}$ described in the proposition also gives this diagram. Hence the two actions are equivalent.

1.21. Special types of actions. Several types of actions are easily understood and recognized from their group diagrams. We will discuss these here so that we can exclude them in our classification.

Product actions. Say $G$ acts on $M$ by cohomogeneity one with group diagram $G \supset K^{-}, K^{+} \supset H$, and $L$ acts transitively on the homogeneous space $L / J$. Then the action of $G \times L$ on $M \times(L / J)$ as a product, that is, $(g, l) \star(p, \ell J)=(g p, l \ell J)$, is cohomogeneity one. Suppose $c$ is a minimal geodesic in $M$ between nonprincipal orbits, which gives the group diagram above. If we fix an $L$-invariant metric on $L / J$, then in the product metric on $M \times(L / J)$ the curve $\tilde{c}=(c, 1)$ is a minimal geodesic between nonprincipal orbits. Clearly, the resulting group diagram is

$$
G \times L \supset K^{-} \times J, K^{+} \times J \supset H \times J
$$

Conversely, any diagram of this form will give a product action as described above. These diagrams are easy to recognize from the $J$ factor that appears in each of the isotropy groups. 
Sum actions. Suppose $G_{i}$ acts transitively, linearly and isometrically on the sphere $S^{m_{i}} \subset \mathbb{R}^{m_{i}+1}$ with isotropy subgroup $H_{i}$, for $i=1,2$. Then we have an action of $G:=G_{1} \times G_{2}$ on $S^{m_{1}+m_{2}+1} \subset \mathbb{R}^{m_{1}+1} \times \mathbb{R}^{m_{2}+1}$ by taking the product action: $\left(g_{1}, g_{2}\right) \star(x, y)=\left(g_{1} \cdot x, g_{2} \cdot y\right)$. Such actions are called sum actions. Now, fix two unit vectors $e_{i} \in S^{m_{i}}$ with $\left(G_{i}\right)_{e_{i}}=H_{i}$, for $i=1,2$, and define $c(\theta)=\left(\cos (\theta) e_{1}, \sin (\theta) e_{2}\right)$. Upon computing the isotropy groups we find that the orbits through $c(\theta)$ for $\theta \in(0, \pi / 2)$ are codimension one and hence this action is cohomogeneity one. We easily find the group diagram to be

$$
G_{1} \times G_{2} \supset G_{1} \times H_{2}, H_{1} \times G_{2} \supset H_{1} \times H_{2} .
$$

Conversely, take a group diagram of this form. Then $G_{i} / H_{i}$ are spheres and hence by the classification of transitive actions on spheres, $G_{i}$ actually acts linearly and isometrically on $S^{m_{i}} \subset \mathbb{R}^{m_{i}+1}$. Hence this action is a sum action as described above. Diagrams of the form (1-6) are easy to recognize from the $H_{1}$ and $H_{2}$ factors in the "middle" and the $G_{1}$ and $G_{2}$ factors on the "outside" of the pair $K^{-}, K^{+}$. In particular these actions are always isometric actions on symmetric spheres.

Fixed point actions. Here we will completely characterize the cohomogeneity one actions that have a fixed point. In fact we will not put any dimension restrictions on the actions in this subsection. Say $G$ acts effectively and by cohomogeneity one on the simply connected manifold $M$ and assume there is a fixed point $p_{-} \in M$, that is, $G_{p_{-}}=G$. It is clear that the point $p_{-}$cannot be in a principal orbit, so we can assume that $K^{-}=G$. Therefore the group diagram for this action will have the form

$$
G \supset G, K^{+} \supset H \text {. }
$$

Conversely, such a diagram clearly gives an action with a fixed point. Therefore to classify fixed point cohomogeneity one actions we must only classify diagrams of type (1-7).

Because we assumed the action is effective, it follows that the $G$ action on $G / H \approx S^{l_{-}}$is an effective transitive action on a sphere. Such actions were classified by Montgomery, Samelson and Borel (see [Besse 1978, page 195]). Up to equivalence, this gives us the possibilities for $G$ and $H$. In particular $H$ and hence $K^{+}$must be connected. Grove and Ziller [2002, Section 2] list all possible closed connected subgroups $K^{+}$between $H$ and $G$ for each pair $G, H$.

In the case where $K^{+}=G$, we have

$$
G \supset G, G \supset H .
$$

To see what this action is, identify $G / H$ with the unit sphere $S^{l} \subset \mathbb{R}^{l+1}$. We know from the classification of transitive actions on spheres mentioned above that $G$ acts 
linearly and isometrically on $\mathbb{R}^{l+1}$. It is easy to check that $M=S^{l+1} \subset \mathbb{R}^{l+1} \times \mathbb{R}$ with the action given by $g \star(x, t)=(g x, t)$. We will call such actions $t w o$-fixedpoint actions. In particular this is an isometric action on the sphere $S^{l+1}$. Notice that if $H_{0}$ is maximal among connected subgroups of $G$, then (1-8) is the only possible diagram for this $G$ and $H_{0}$, assuming there are no exceptional orbits. This gives the following convenient proposition.

Proposition 1.22. Let $M$ be a simply connected cohomogeneity one manifold for the group $G$, with principal isotropy group $H$, as above. If $H_{0}$ is maximal among connected subgroups of $G$, then the action is equivalent to an isometric two-fixedpoint action on a sphere.

Therefore we must only consider the case in which $K^{+}$is a subgroup strictly between $H$ and $G$. Following the tables given in [Grove and Ziller 2002], we address these cases one by one. We first list the diagram, then the corresponding action. In each case it is easy to check that the action listed gives the corresponding diagram.

- $\mathrm{SU}(n) \supset \mathrm{SU}(n), \mathrm{S}(\mathrm{U}(n-1) \mathrm{U}(1)) \supset \mathrm{SU}(n-1)$ :

$\mathrm{SU}(n)$ on $\mathbb{C P}^{n}$ given by $A \star\left[z_{0}, z_{1}, \ldots, z_{n}\right]=\left[z_{0}, A\left(z_{1}, \ldots, z_{n}\right)\right]$.

- $\mathrm{U}(n) \supset \mathrm{U}(n), \mathrm{U}(n-1) \mathrm{U}(1) \supset \mathrm{U}(n-1)$ :

$\mathrm{U}(n)$ on $\mathbb{C P}^{n}$ given by $A \star\left[z_{0}, z_{1}, \ldots, z_{n}\right]=\left[z_{0}, A\left(z_{1}, \ldots, z_{n}\right)\right]$.

- $\operatorname{Sp}(n) \supset \operatorname{Sp}(n), \operatorname{Sp}(n-1) \operatorname{Sp}(1) \supset \operatorname{Sp}(n-1)$ :

$\operatorname{Sp}(n)$ on $\mathbb{H} \mathbb{P}^{n}$ given by $A \star\left[x_{0}, x_{1}, \ldots, x_{n}\right]=\left[x_{0}, A\left(x_{1}, \ldots, x_{n}\right)\right]$.

- $\operatorname{Sp}(n) \supset \operatorname{Sp}(n), \operatorname{Sp}(n-1) \mathrm{U}(1) \supset \operatorname{Sp}(n-1)$ :

$\operatorname{Sp}(n)$ on $\mathbb{C} \mathbb{P}^{2 n+1}=S^{4 n+3} / S^{1}$ for $S^{4 n+3} \subset \mathbb{H}^{n+1}$ given by

$$
A \star\left[x_{0}, x_{1}, \ldots, x_{n}\right]=\left[x_{0}, A\left(x_{1}, \ldots, x_{n}\right)\right]
$$

- $\operatorname{Sp}(n) \times \operatorname{Sp}(1) \supset \operatorname{Sp}(n) \times \operatorname{Sp}(1), \operatorname{Sp}(n-1) \operatorname{Sp}(1) \times \operatorname{Sp}(1) \supset \operatorname{Sp}(n-1) \Delta \operatorname{Sp}(1)$ : $\operatorname{Sp}(n) \times \operatorname{Sp}(1)$ on $\mathbb{M} \mathbb{P}^{n}$ given by

$$
(A, p) \star\left[x_{0}, x_{1}, \ldots, x_{n}\right]=\left[p x_{0}, A\left(x_{1}, \ldots, x_{n}\right)\right] .
$$

- $\operatorname{Sp}(n) \times \mathrm{U}(1) \supset \mathrm{Sp}(n) \times \mathrm{U}(1), \operatorname{Sp}(n-1) \operatorname{Sp}(1) \times \mathrm{U}(1) \supset \mathrm{Sp}(n-1) \Delta \mathrm{U}(1)$ : $\mathrm{Sp}(n) \times \mathrm{U}(1)$ on $\mathbb{H} \mathbb{P}^{n}$ given by

$$
(A, z) \star\left[x_{0}, x_{1}, \ldots, x_{n}\right]=\left[z x_{0}, A\left(x_{1}, \ldots, x_{n}\right)\right] .
$$

- $\mathrm{Sp}(n) \times \mathrm{U}(1) \supset \mathrm{Sp}(n) \times \mathrm{U}(1), \mathrm{Sp}(n-1) \mathrm{U}(1) \times \mathrm{U}(1) \supset \mathrm{Sp}(n-1) \Delta \mathrm{U}(1)$ : $\mathrm{Sp}(n) \times \mathrm{U}(1)$ on $\mathbb{C} \mathbb{P}^{2 n+1}=S^{4 n+3} / S^{1}$ for $S^{4 n+3} \subset \mathbb{H}^{n+1}$ given by

$$
(A, z) \star\left[x_{0}, x_{1}, \ldots, x_{n}\right]=\left[z x_{0}, A\left(x_{1}, \ldots, x_{n}\right)\right] .
$$

- $\operatorname{Spin}(9) \supset \operatorname{Spin}(9), \operatorname{Spin}(8) \supset \operatorname{Spin}(7)$ :

$\operatorname{Spin}(9)$ on $\mathrm{CaP}^{2}=\mathrm{F}_{4} / \operatorname{Spin}(9)$; see [Iwata 1981].

In conclusion, we have shown the following. 
Proposition 1.23. Every cohomogeneity one action on a compact simply connected manifold with a fixed point is equivalent to one of the isometric actions on a compact rank one symmetric space described above.

1.24. Important Lie groups. It is well known that every compact connected Lie group has a finite cover of the form $G_{s s} \times T^{k}$, where $G_{s s}$ is semisimple and simply connected and $T^{k}$ is a torus. The classification of compact simply connected semisimple Lie groups is also well known. In dimensions 21 and less, such a group must be a product of the following: the 3-dimensional $S^{3} \approx \mathrm{SU}(2) \approx \mathrm{Sp}(1) \approx$ Spin(3) with rank 1; the 8-dimensional SU(3), the 10-dimensional Sp(2) $\approx \operatorname{Spin}(5)$ and 14-dimensional $\mathrm{G}_{2}$, all with rank 2 ; and the 15-dimensional $\mathrm{SU}(4) \approx \operatorname{Spin}(6)$, the 21-dimensional $\operatorname{Sp}(3)$ and the 21-dimensional Spin(7), all with rank 3.

If an arbitrary compact group $G$ acts on a manifold $M$, then every cover $\tilde{G}$ of $G$ still acts on $M$, although less effectively. So allowing for a finite ineffective kernel, and since $G$ will always have dimension 21 or less in our case by Proposition 1.19, we can assume that $G$ is the product of groups listed above, together with a torus $T^{k}$.

For the classifications of cohomogeneity one diagrams we will also need to know the subgroups of the groups listed above, for certain dimensions. These subgroups are well known; see for example [Dynkin 1952]. For the above groups, we will now list the proper connected closed subgroups in the dimensions that will be relevant for our study, for future reference. In $T^{2}$ we have only $\left\{\left(e^{i p \theta}, e^{i q \theta}\right)\right\}$ and for $S^{3}$ we only have $\left\{e^{x \theta}=\cos \theta+x \sin \theta\right\}$ where $x \in \operatorname{Im}\left(S^{3}\right)$. In $S^{3} \times S^{3}$ we have $S^{1} \subset T^{2}$, $T^{2}, S^{3} \times 1,1 \times S^{3}, \Delta S^{3}=\{(g, g)\}, S^{3} \times S^{1}$, and $S^{1} \times S^{3}$. The group SU(3) has $S^{1} \subset T^{2}, T^{2}, \mathrm{SO}(3), \mathrm{SU}(2)$, and $\mathrm{U}(2)=\mathrm{S}(\mathrm{U}(2) \mathrm{U}(1))$. For $\mathrm{Sp}(2)$, in dimensions 4 and higher we have $\mathrm{U}(2), \operatorname{Sp}(1) \mathrm{SO}(2)$, and $\mathrm{Sp}(1) \mathrm{Sp}(1)$. In dimensions 8 or greater $\mathrm{SU}(3)$ is the only such subgroup of $\mathrm{G}_{2}$. And finally $\mathrm{SU}(4)$ has the subgroups $\mathrm{U}(3)$ and $\operatorname{Sp}(2)$ in dimensions 9 or higher.

We can use this information about the subgroups of the classical Lie groups to make the following claim.

Proposition 1.25. Let $M$ be the cohomogeneity one manifold given by the group diagram $G \supset K^{+}, K^{-} \supset H$, where $G$ acts nonreducibly on $M$. Suppose $G$ is the product of groups

$$
G=\prod_{t=1}^{i}(\mathrm{SU}(4)) \times \prod_{t=1}^{j}\left(\mathrm{G}_{2}\right) \times \prod_{t=1}^{k}(\mathrm{Sp}(2)) \times \prod_{t=1}^{l}(\mathrm{SU}(3)) \times \prod_{t=1}^{m}\left(S^{3}\right) \times\left(S^{1}\right)^{n}
$$

where $i, j, k, l, m$ and $n$ are allowed to be zero and where we imagine most of them are zero. Then

$$
\operatorname{dim}(H) \leq 10 i+8 j+6 k+4 l+m .
$$


Of course the most important applications of this proposition will be in the case that $i, j, k, l, m$ and $n$ are all small and mostly zero. Although this might not seem to be of much use, we will see that it will help to rule out many product groups.

Proof. Since the action is nonreducible, we know that $H$ does not project onto any of the factors in this product. That means each $\operatorname{proj}_{v}(H)$ is a proper subgroup. Therefore

$$
H_{0} \subset \prod_{t=1}^{i}\left(I_{t}\right) \times \prod_{t=1}^{j}\left(J_{t}\right) \times \prod_{t=1}^{k}\left(K_{t}\right) \times \prod_{t=1}^{l}\left(L_{t}\right) \times \prod_{t=1}^{m}\left(S_{t}^{1}\right) \times 1 .
$$

We listed the largest possible dimension of each of these subgroups at the beginning of this section. In particular

$$
\operatorname{dim}\left(I_{t}\right) \leq 10, \quad \operatorname{dim}\left(J_{t}\right) \leq 8, \quad \operatorname{dim}\left(K_{t}\right) \leq 6, \quad \operatorname{dim}\left(L_{t}\right) \leq 4 .
$$

\section{Classification in dimension five}

Throughout this section, $M$ will denote a 5-dimensional compact simply connected cohomogeneity one manifold for the compact connected group $G$ that acts almost effectively and nonreducibly, with group diagram $G \supset K^{-}, K^{+} \supset H$, where $K^{ \pm} / H \approx S^{l_{ \pm}}$.

We will complete the classification by finding all such group diagrams that give simply connected manifolds. The first step is to find the possibilities for $G$. Since we allow the action to have finite ineffective kernel, after lifting the action to a covering group of $G$, we can assume $G$ is a product of groups from Section 1.24. In fact we have the following proposition.

Proposition 2.1. $G$ and $H_{0}$ must be one of the pairs of groups listed in Table III, up to equivalence.

Proof. We will first show that all the possibilities for $G$ are listed in the table. We know from Proposition 1.19 that $4 \leq \operatorname{dim} G \leq 10$ and $\operatorname{dim} H=\operatorname{dim} G-4$ since the principal orbits $G / H$ are codimension one in $M$. Further, since $G$ is a product of groups from Section 1.24, $G$ must have the form $\left(S^{3}\right)^{m} \times T^{n}$, SU(3) $\times T^{n}$ or Spin(5). From Proposition 1.18 we can assume $n \leq 2$ in all cases. First suppose that $G=\left(S^{3}\right)^{m} \times T^{n}$. Then by Proposition 1.25 we have $3 m+n-4=\operatorname{dim} H \leq m$, which means $0 \leq 4-2 m-n$. Hence $m \leq 2$ and if $m=2$ then $n=0$. So all the possibilities for groups of the form $\left(S^{3}\right)^{m} \times T^{n}$ are in fact listed in the table. Next suppose $G=\mathrm{SU}(3) \times T^{n}$. Then by Proposition 1.25 again we know that $\operatorname{dim} H \leq 4$, which means $\operatorname{dim} G=\operatorname{dim} H+4 \leq 8$. Hence $\mathrm{SU}(3)$ is the only possibility of this form. Therefore all of the possible groups $G$ are listed in Table III.

Now we will show that for each possible $G$ described above we have listed all the possible subgroups $H_{0}$ of the right dimension. It is clear that if $G=S^{3} \times S^{1}$, 


\begin{tabular}{ccc}
\hline Case & $G$ & $H_{0}$ \\
\hline $1_{5}$ & $S^{3} \times S^{1}$ & $\{1\}$ \\
$2_{5}$ & $S^{3} \times T^{2}$ & $S^{1} \times 1$ \\
$3_{5}$ & $S^{3} \times S^{3}$ & $T^{2}$ \\
$4_{5}$ & $\mathrm{SU}(3)$ & $\mathrm{U}(2)$ \\
$5_{5}$ & Spin(5) & Spin(4) \\
\hline
\end{tabular}

Table III. Possibilities for $G$ and $H_{0}$ in the 5-dimensional case.

then $H$ is discrete. Next, if $G=S^{3} \times T^{2}$, then for the action to be nonreducible, $\operatorname{proj}_{2}(H) \subset T^{2}$ must be trivial. Hence, $H_{0}$ is a closed connected one-dimensional subgroup of $S^{3}$, as stated. If $G=S^{3} \times S^{3}$, then it is clear that $T^{2}$ must be a maximal torus in $G$. If $G=\mathrm{SU}(3)$, we see from Section 1.24 that $H_{0}$ must be $\mathrm{U}(2)$ up to conjugation. Finally, Proposition 1.20 deals with the last case $\operatorname{dim} G=10$.

In the rest of the section we proceed case by case to find all possible diagrams for the pairs of groups listed in Table III. We will do this by finding the possibilities for $K^{ \pm}$, with $K^{ \pm} / H$ a sphere. Recall from Propositions 1.7 and 1.23 that we can assume

$$
\operatorname{dim} G>\operatorname{dim} K^{ \pm}>\operatorname{dim} H .
$$

Case $1_{5}\left(G=S^{3} \times S^{1}\right)$. In this case $H$ must be discrete. It then follows that for $K / H$ to be a sphere, $K_{0}$ itself must be a cover of a sphere. Then from Section 1.24, the only compact connected subgroups of $S^{3} \times S^{1}$ that cover spheres are $S^{3} \times 1$ or circle groups of the form $\left\{\left(e^{x p \theta}, e^{i q \theta}\right)\right\}$ where $x \in \operatorname{Im}(\mathbb{U})$. From Proposition 1.18, we know that at least one of $K_{0}^{ \pm}$is a circle. This leads us into the following cases: both $K_{0}^{ \pm}$are circles or $K_{0}^{-}$is a circle and $K_{0}^{+}=S^{3} \times 1$.

Case $1_{5} \mathrm{~A}\left(K_{0}^{-}\right.$is a circle and $\left.K_{0}^{+}=S^{3} \times 1\right)$. First, from Corollary $1.9, K^{-}$must be connected with $H \subset K^{-}$and $H \cap K_{0}^{+}=1$. After conjugation of $G$, we may assume $K^{-}=\left\{\left(e^{i p \theta}, e^{i q \theta}\right)\right\}$ and $p, q \geq 0$. We also know from Corollary 1.9 that for $M$ to be simply connected, $G / K^{-}=S^{3} \times S^{1} /\left\{\left(e^{i p \theta}, e^{i q \theta}\right)\right\}$ must also be simply connected. It is not hard to see that this happens precisely when $q=1$. Finally, if $H=\mathbb{Z}_{n} \subset K^{-}$the condition that $H \cap\left(1 \times S^{1}\right)=e$ means $(p, n)=1$. Then $K^{+}=K_{0}^{+} \cdot H=\left(S^{3} \times 1\right) \cdot \mathbb{Z}_{n}=S^{3} \times \mathbb{Z}_{n}$. In conclusion, such an action must have the following type of group diagram:

$$
S^{3} \times S^{1} \supset\left\{\left(e^{i p \theta}, e^{i \theta}\right)\right\},\left(S^{3} \times 1\right) \cdot \mathbb{Z}_{n} \supset \mathbb{Z}_{n}
$$

Conversely, such groups clearly determine a simply connected cohomogeneity one manifold, by Proposition 1.8. This family $Q_{C}^{5}$ is described in more detail in Section 5.1. 
Case $1_{5} \mathbf{B}$ (both $K_{0}^{ \pm}$are circle groups). After conjugation we can take

$$
K_{0}^{-}=\left\{\left(e^{i p_{-} \theta}, e^{i q_{-} \theta}\right)\right\} \quad \text { and } \quad K_{0}^{+}=\left\{\left(e^{x p_{+} \theta}, e^{i q_{+} \theta}\right)\right\}
$$

for some $x \in \operatorname{Im}(\mathbb{M}) \cap S^{3}$ and $\left(p_{ \pm}, q_{ \pm}\right)=1$. From Lemma 1.10, we know that $H$ must be generated by $H_{-}=H \cap K_{0}^{-}$and $H_{+}=H \cap K_{0}^{+}$, which are cyclic subgroups of the circles $K_{0}^{-}$and $K_{0}^{+}$, respectively.

We will now have to break this into two more cases, depending on whether $K_{0}^{-}$ and $K_{0}^{+}$are both contained in a torus $T^{2}$ in $G$.

Case $1_{5} \mathbf{B 1}\left(K_{0}^{-}\right.$and $K_{0}^{+}$are not both contained in any torus $\left.T^{2} \subset G\right)$. Here we can assume that $x \neq \pm i$. Further, from (2-2), we see $p_{ \pm} \neq 0$ in this case, since otherwise $K_{0}^{ \pm}$would be contained in the same torus. Further, from Proposition 1.18, we know that at least one of $q_{ \pm}$must be nonzero, say $q_{+} \neq 0$. A computation shows that $N\left(K_{0}^{+}\right)=\left\{\left(e^{x \theta}, e^{i \phi}\right)\right\}$, since $p_{+} q_{+} \neq 0$. Therefore $K^{+} \subset\left\{\left(e^{x \theta}, e^{i \phi}\right)\right\}$, since every compact subgroup of a Lie group is contained in the normalizer of its identity component. Similarly,

$$
K^{-} \subset N\left(K_{0}^{-}\right)= \begin{cases}\left\{\left(e^{i \theta}, e^{i \phi}\right)\right\} & \text { if } q_{-} \neq 0, \\ \left\{\left(e^{i \theta}, e^{i \phi}\right)\right\} \cup\left\{\left(j e^{i \theta}, e^{i \phi}\right)\right\} & \text { if } q_{-}=0 .\end{cases}
$$

Therefore $H \subset K^{-} \cap K^{+} \subset N\left(K_{0}^{-}\right) \cap N\left(K_{0}^{+}\right)$. If $q_{-} \neq 0$, then this means $H \subset\left\{\left(e^{i \theta}, e^{i \phi}\right)\right\} \cap\left\{\left(e^{x \theta}, e^{i \phi}\right)\right\}=\left\{\left( \pm 1, e^{i \phi}\right)\right\}$. Then $H$ lies in the center of $G$ and so by Proposition 1.2, we can conjugate $K^{+}$by any element of $G$ and still have the same action. In particular we can conjugate $K^{+}$to lie in the same torus as $K^{-}$, hence reducing such actions to Case $1_{5} \mathrm{~B} 2$. So we can assume that $q_{-}=0$ and hence $K_{0}^{-}=\left\{\left(e^{i \theta}, 1\right)\right\}$.

Then, we have

$$
H \subset N\left(K_{0}^{-}\right) \cap N\left(K_{0}^{+}\right)=\left(\left\{\left(e^{i \theta}, e^{i \phi}\right)\right\} \cup\left\{\left(j e^{i \theta}, e^{i \phi}\right)\right\}\right) \cap\left\{\left(e^{x \theta}, e^{i \phi}\right)\right\} .
$$

This intersection will again be $\left\{\left( \pm 1, e^{i \phi}\right)\right\}$ unless $x \perp i$. As above, we can again assume $x \perp i$. Further, after conjugation of $G$ by $\left(e^{i \theta_{0}}, 1\right)$ for a certain value of $\theta_{0}$, $K_{0}^{-}$will remain fixed and $K_{0}^{+}$will be taken to $\left\{\left(e^{j p_{+} \theta}, e^{i q_{+} \theta}\right)\right\}$, with $p_{+}, q_{+}>0$. So we can assume

$$
K_{0}^{-}=\left\{\left(e^{i \theta}, 1\right)\right\} \quad \text { and } \quad K_{0}^{+}=\left\{\left(e^{j p_{+} \theta}, e^{i q_{+} \theta}\right)\right\} .
$$

and therefore, $H \subset N\left(K_{0}^{-}\right) \cap N\left(K_{0}^{+}\right)=\{ \pm 1, \pm j\} \times S^{1} \subset S^{3} \times S^{1}$. We saw above that we can assume $H$ is not contained in $\left\{\left( \pm 1, e^{i \phi}\right)\right\}$, and hence $H$ must contain an element of the form $\left(j, z_{0}\right)$, which we can assume also lies in $K_{0}^{+}$, by Lemma 1.10. We can also assume that $H \cap\left(1 \times S^{1}\right)=1$, so that $\#\left(z_{0}\right) \mid \#(j)=4$ and hence $z_{0} \in\{ \pm 1, \pm i\}$, where \# $(g)$ denotes the order of the element $g$. So $H \cap K_{0}^{+}$ is generated by $\left(j, z_{0}\right)$. Similarly, $H \cap K_{0}^{-}$must also be a subset of $\{ \pm 1, \pm j\} \times 1$ 
and is therefore either trivial or $\{( \pm 1,1)\}$. For convenience we break this up into three more cases, depending on the order of $z_{0}$.

Case $1_{5} \mathbf{B 1 a}$ (the order of $z_{0}$ is one, that is, $z_{0}=1$ ). In this case $H=\langle(j, 1)\rangle$, $K_{0}^{-} \cap H=\{( \pm 1,1)\}$ and $K_{0}^{+} \cap H=H$. Hence $K^{+}$is connected. The condition that $H \subset K^{+}$means $4 \mid q_{+}$and $p_{+}$is odd. We can represent $\pi_{1}\left(K^{+} / H\right)$ with the curve $\alpha_{+}:[0,1] \rightarrow K^{+}, t \mapsto\left(e^{2 \pi j p_{+} t / 4}, e^{2 \pi i q_{+} t / 4}\right)$, and we can represent $\pi_{1}\left(K^{-} / H\right)$ with the curve $\alpha_{-}:[0,1] \rightarrow K^{-}, t \mapsto\left(e^{2 \pi i t / 2}, 1\right)$. From Lemma $1.10, M$ will be simply connected if and only if $\alpha_{ \pm}$generate $\pi_{1}(G)$. We see that the possible loops in $G$ that $\alpha_{ \pm}$can form are combinations of $\alpha_{-}^{2}, \alpha_{+}^{4}$ and $\alpha_{-} \circ \alpha_{+}^{2}$. Yet each of these loops can only give an even multiple of the loop $1 \times S^{1} \subset S^{3} \times S^{1}=G$, which generates $\pi_{1}(G)$. Hence $M$ will never be simply connected in this case.

Case $1{ }_{5} \mathbf{B} 1 \mathbf{b}$ (the order of $z_{0}$ is two, that is, $z_{0}=-1$ ). In this case $H=\langle(j,-1)\rangle$, and again $K_{0}^{-} \cap H=\{( \pm 1,1)\}$ and $K_{0}^{+} \cap H=H$, so that $K^{+}$is connected. This time, the condition that $H \subset K^{+}$means that $p_{+}$is odd and $q_{+} \equiv 2 \bmod 4$. Then, we can represent $\pi_{1}\left(K^{+} / H\right)$ with the curve $\alpha_{+}:[0,1] \rightarrow K^{+}$mapping $t \mapsto\left(e^{2 \pi j p_{+} t / 4}, e^{2 \pi i q_{+} t / 4}\right)$ and $\pi_{1}\left(K^{-} / H\right)$ with the curve $\alpha_{-}:[0,1] \rightarrow K^{-}$mapping $t \mapsto\left(e^{2 \pi i t / 2}, 1\right)$, and again $M$ will be simply connected if and only if $\alpha_{ \pm}$generate $\pi_{1}(G)$, by Lemma 1.10. The loops that $\alpha_{ \pm}$can generate are again combinations of $\alpha_{-}^{2}, \alpha_{+}^{4}$ and $\alpha_{-} \circ \alpha_{+}^{2}$ but in this case $\alpha_{-}^{2}$ corresponds to zero times around the loop $1 \times S^{1}, \alpha_{+}^{4}$ corresponds to $q_{+}$times around $1 \times S^{1}$, and $\alpha_{-} \circ \alpha_{+}^{2}$ corresponds to $q_{+} / 2$ times around $1 \times S^{1}$. Together with the constraints $q_{+} \equiv 2 \bmod 4$ and $q_{+}>0$, we see that $M$ will be simply connected if and only if $q_{+}=2$. Therefore this case gives this family of actions:

$\left(Q_{B}^{5}\right) \quad S^{3} \times S^{1} \supset\left\{\left(e^{i \theta}, 1\right)\right\} \cdot H,\left\{\left(e^{j p_{+} \theta}, e^{2 i \theta}\right)\right\} \supset\langle(j,-1)\rangle, \quad$ where $p_{+}>0$ is odd.

These actions are described in more detail in Section 5.2.

Case $1_{5} \mathrm{B1C}$ (the order of $z_{0}$ is four, that is, $z_{0}= \pm i$ ). After a conjugation of $G$, which will not effect the form of $K^{ \pm}$, we can assume $z_{0}=i$, that is, $(j, i) \in H \cap K_{0}^{+}$, although we can no longer assume $p_{+}>0$. As above, $H \cap K_{0}^{-} \subset\{( \pm 1,1)\}$. Yet if $(-1,1) \in H$, then $(-1,-1) \cdot(-1,1)=(1,-1) \in H$, violating our assumption that $H \cap 1 \times S^{1}=1$. Therefore $H=\langle(j, i)\rangle \subset K^{+}, K^{+}$is connected and $K_{0}^{-} \cap H=1$. This also implies that $p_{+}$and $q_{+}$are odd and $p_{+} \equiv q_{+} \bmod 4$. In this case $\pi_{1}\left(K^{+} / H\right)$ can be represented by the curve $\alpha_{+}:[0,1] \rightarrow K^{+}$mapping $t \mapsto\left(e^{2 \pi j p_{+} t / 4}, e^{2 \pi i q_{+} t / 4}\right)$ and $\pi_{1}\left(K^{-} / H\right)$ can be represented by $\alpha_{-}:[0,1] \rightarrow K^{-}$, $t \mapsto\left(e^{2 \pi i t}, 1\right)$. As above, by Lemma $1.10, M$ will be simply connected if and only if the $\alpha_{ \pm}$generate $\pi_{1}(G)$. We see that the only loops in $G$ that $\alpha_{ \pm}$can generate are $\alpha_{-}$and $\alpha_{+}^{4}$, where $\alpha_{-}$in trivial in $\pi_{1}(G)$ and $\alpha_{+}^{4}$ represents $q_{+}$times around the loop $1 \times S^{1}$, which generates $\pi_{1}(G)$. Together with our assumption that $q_{+}>0$, we get that $M$ is simply connected if and only if $q_{+}=1$. This case gives precisely 
the family $P^{5}$ of actions (from the introduction):

$$
\left(P^{5}\right) \quad S^{3} \times S^{1} \supset\left\{\left(e^{i \theta}, 1\right)\right\} \cdot H,\left\{\left(e^{j p_{+} \theta}, e^{i \theta}\right)\right\} \supset\langle(j, i)\rangle \quad \text { where } p_{+} \equiv 1 \bmod 4 .
$$

Case $1_{5} \mathbf{B 2}\left(K_{0}^{-}\right.$and $K_{0}^{+}$are both contained in a torus $\left.T^{2} \subset G\right)$. After conjugation of $G$, we may assume that $K_{0}^{ \pm} \subset\left\{\left(e^{i \theta}, e^{i \phi}\right)\right\}$. It then follows from Lemma 1.10 that $K^{ \pm}, H \subset\left\{\left(e^{i \theta}, e^{i \phi}\right)\right\}$. Here again there will be two cases depending on whether or not $K_{0}^{-}$and $K_{0}^{+}$are distinct circles.

Case $1_{5} \mathbf{B 2 a}\left(K_{0}^{-}=K_{0}^{+}\right)$. Then we can take $K_{0}^{-}=K_{0}^{+}=\left\{\left(e^{i p \theta}, e^{i q \theta}\right)\right\}$ with $q>0$. From Lemma 1.10, it follows that $H$ is a cyclic subgroup of $K_{0}^{ \pm}$and that $K^{ \pm}$is connected. It is then clear from Lemma 1.10 that $q=1$ and hence we have this family of actions:

$$
S^{3} \times S^{1} \supset\left\{\left(e^{i p \theta}, e^{i \theta}\right)\right\},\left\{\left(e^{i p \theta}, e^{i \theta}\right)\right\} \supset \mathbb{Z}_{n} .
$$

Conversely, the resulting manifolds will all be simply connected by Lemma 1.10.

Case $1_{5} \mathbf{B} 2 \mathbf{b}\left(K_{0}^{-} \neq K_{0}^{+}\right)$. Here, say $K_{0}^{ \pm}=\left\{\left(e^{i p_{ \pm} \theta}, e^{i q_{ \pm} \theta}\right)\right\}$ and then $H=H_{-} \cdot H_{+}$ for cyclic subgroups $H_{ \pm} \subset K_{0}^{ \pm}$, by Lemma 1.10. Now let $\alpha_{ \pm}:[0,1] \rightarrow K_{0}^{ \pm}$be curves with $\alpha_{ \pm}(0)=1$ that represent $\pi_{1}\left(K^{ \pm} / H\right)$. Then by Lemma $1.10, M$ will be simply connected if and only if the combinations of $\alpha_{ \pm}$that form loops in $G$ generate $\pi_{1}(G)$. Let $\delta_{ \pm}:[0,1] \rightarrow K_{0}^{ \pm}, t \mapsto\left(e^{2 \pi i p_{ \pm} t}, e^{2 \pi i q_{ \pm} t}\right)$ be curves that pass once around the circles $K_{0}^{ \pm}$. Then $\delta_{ \pm}=\alpha_{ \pm}^{m_{ \pm}}$are two such loops.

To find all such loops, consider the covering map $\wp: \mathbb{R}^{2} \rightarrow T^{2} \subset S^{3} \times S^{1}$, $(x, y) \mapsto\left(e^{2 \pi i x}, e^{2 \pi i y}\right)$ and let $\widetilde{K}^{ \pm}$be the line through the origin and through the point $\left(p_{ \pm}, q_{ \pm}\right)$in $\mathbb{R}^{2}$. Then it is clear that $\wp^{-1}\left(K_{0}^{ \pm}\right)=\widetilde{K}^{ \pm}+\mathbb{Z}^{2}$, the discrete collection of lines in $\mathbb{R}^{2}$ that are parallel to $\widetilde{K}^{ \pm}$and that pass through an integer lattice point.

Now let $\tilde{\gamma}$ be a path in $\mathbb{R}^{2}$ that starts at $(0,0)$ follows $\widetilde{K}^{-}$until the first point of the intersection $\widetilde{K}^{-} \cap\left(\widetilde{K}^{+}+\mathbb{Z}^{2}\right)$, then follows $\widetilde{K}^{+}+\mathbb{Z}^{2}$ to the first integer lattice point $(\lambda, \mu)$. Then $\gamma:=\wp(\tilde{\gamma})$ gives a loop in $G$. Notice that $K_{0}^{-} \cap K_{0}^{+}$is a cyclic subgroup of both $K_{0}^{ \pm}$, and any curve in $K_{0}^{-} \cup K_{0}^{+}$is homotopic within $T^{2}$ to a curve in $K_{0}^{-}$followed by a curve in $K_{0}^{+}$. It then follows that $\delta_{-}, \delta_{+}$and $\gamma$ generate all possible loops in $K_{0}^{-} \cup K_{0}^{+}$. Similarly, if $d$ is the index of $H \cap K_{0}^{-} \cap K_{0}^{+}$in $K_{0}^{-} \cap K_{0}^{+}$, then $\gamma^{d}$ can be imagined as a curve that starts at 1 , travels along $K_{0}^{-}$to the first element of $H$ in $H \cap K_{0}^{-} \cap K_{0}^{+}$, and then follows $K_{0}^{+}$back to the identity. Then $\delta_{-}, \delta_{+}$and $\gamma^{d}$ generate the same homotopy classes of loops as $\alpha_{-}$and $\alpha_{+}$. Therefore $M$ will be simply connected if and only if $\delta_{-}, \delta_{+}$and $\gamma^{d}$ generate $\pi_{1}(G)$.

Let $c:[0,1] \rightarrow G=S^{3} \times S^{1}, t \mapsto\left(1, e^{2 \pi i t}\right)$ represent the generator of $\pi_{1}(G)$. Then it is clear that $\delta_{ \pm}$is homotopic to $c^{q_{ \pm}}$in $G$ and that $\gamma$ is homotopic to $c^{\mu}$ in $G$. Therefore $M$ is simply connected if and only if $\left\langle c^{q_{-}}, c^{q_{+}}, c^{d \mu}\right\rangle=\langle c\rangle$. Notice further that there are no integer lattice points strictly between the lines $\widetilde{K}^{+}$and 
$(\lambda, \mu)+\widetilde{K}^{+}$since there are no lines of $\widetilde{K}^{+}+\mathbb{Z}^{2}$ in this region, by the construction of $\tilde{\gamma}$. Therefore $\left(p_{+}, q_{+}\right)$and $(\lambda, \mu)$ generate all of $\mathbb{Z}^{2}$ and in particular $q_{+}$and $\mu$ are relatively prime. Hence $\left\langle c^{q_{-}}, c^{q_{+}}, c^{d \mu}\right\rangle=\left\langle c^{q_{-}}, c^{q_{+}}, c^{d}\right\rangle$ and therefore $M$ is simply connected if and only if $\operatorname{gcd}\left(q_{-}, q_{+}, d\right)=1$. Therefore, we get precisely the family $N^{5}$ of simply connected diagrams:

$$
\begin{aligned}
&\left(N^{5}\right) \quad S^{3} \times S^{1} \supset\left\{\left(e^{i p_{-} \theta}, e^{i q_{-} \theta}\right)\right\} \cdot H,\left\{\left(e^{i p_{+} \theta}, e^{i q_{+} \theta}\right)\right\} \cdot H \supset H_{-} \cdot H_{+} \\
& K^{-} \neq K^{+}, \operatorname{gcd}\left(q_{-}, q_{+}, d\right)=1, \quad \text { where } d=\#\left(K_{0}^{-} \cap K_{0}^{+}\right) / \#\left(H \cap K_{0}^{-} \cap K_{0}^{+}\right) .
\end{aligned}
$$

The referee pointed out that the condition $\operatorname{gcd}\left(q_{-}, q_{+}, d\right)=1$ can be replaced by either condition $\operatorname{gcd}\left(q_{-}, d\right)=1$ or $\operatorname{gcd}\left(q_{+}, d\right)=1$. Notice that $d \mid \#\left(K_{0}^{-} \cap K_{0}^{+}\right)=$ $\left|p_{-} q_{+}-p_{+} q_{-}\right|$. Therefore $\operatorname{gcd}\left(d, q_{+}\right)$divides both $p_{-} q_{+}-p_{+} q_{-}$and $p_{-} q_{+}$and hence $\operatorname{gcd}\left(d, q_{+}\right) \mid p_{+} q_{-}$. Therefore $\operatorname{gcd}\left(d, q_{+}\right) \mid q_{-}$since $\operatorname{gcd}\left(p_{+}, q_{+}\right)=1$. In particular $\operatorname{gcd}\left(q_{-}, q_{+}, d\right)=\operatorname{gcd}\left(q_{+}, d\right)$ and similarly $\operatorname{gcd}\left(q_{-}, q_{+}, d\right)=\operatorname{gcd}\left(q_{-}, d\right)$.

We next examine the remaining possibilities for $G$ : Cases $25_{5}-5$ from Table III. Cases $2_{5}, 4_{5}$ and 5. In Case $2_{5}, G=S^{3} \times T^{2}$ and $H_{0}=S^{1} \times 1$ where rank $S^{3}=$ rank $S^{1}$. Proposition 1.18 then says that the resulting action must be a product action. In Case 45 , we know from Section 1.24 that $H_{0}=\mathrm{U}(2)$ is maximal among connected subgroups of $G=\mathrm{SU}(3)$. Hence any action with these groups would be an isometric two-fixed-point action on a sphere, by Proposition 1.22. Finally, Case $5_{5}$ is fully described by Proposition 1.20.

Case 3. Now $G=S^{3} \times S^{3}$ and $H_{0}=S^{1} \times S^{1}$. Then from Section 1.24, any proper connected subgroup $K$ of $G$, containing $H_{0}$ and of higher dimension, must be $S^{3} \times S^{1}$ or $S^{1} \times S^{3}$. Then, since our only possibilities for $K$ have $\operatorname{dim}(K / H)=2$, Corollary 1.9 implies that all of $H, K^{-}$and $K^{+}$are connected. Therefore, up to equivalence, we only have the following possible diagrams:

$$
\begin{aligned}
& S^{3} \times S^{3} \supset S^{3} \times S^{1}, S^{3} \times S^{1} \supset S^{1} \times S^{1}, \\
& S^{3} \times S^{3} \supset S^{3} \times S^{1}, S^{1} \times S^{3} \supset S^{1} \times S^{1} .
\end{aligned}
$$

Conversely, it is clear that these both give simply connected manifolds. The first of these actions is a product action and the second is a sum action.

\section{Classification in dimension six}

Throughout this section we will keep the notations and conventions established at the beginning of Section 2, this time for a 6-dimensional manifold $M$. As in the previous case we have the following result to describe the possible groups.

Proposition 3.1. $G$ and $H_{0}$ must be one of the pairs of groups listed in Table IV, up to equivalence. 


\begin{tabular}{ccc}
\hline Case & $G$ & $H_{0}$ \\
\hline $1_{6}$ & $S^{3} \times T^{2}$ & $\{1\}$ \\
$2_{6}$ & $S^{3} \times S^{3}$ & $\left\{\left(e^{i p \theta}, e^{i q \theta}\right)\right\}$ \\
$3_{6}$ & $S^{3} \times S^{3} \times S^{1}$ & $T^{2} \times 1$ \\
$4_{6}$ & $\mathrm{SU}(3)$ & $\mathrm{SU}(2), \mathrm{SO}(3)$ \\
$5_{6}$ & $\mathrm{SU}(3) \times S^{1}$ & $\mathrm{U}(2) \times 1$ \\
$6_{6}$ & $\mathrm{Sp}(2) \times S^{1}$ & $\mathrm{Sp}(1) \mathrm{Sp}(1) \times 1$ \\
$7_{6}$ & $\mathrm{Spin}(6)$ & $\mathrm{Spin}(5)$ \\
\hline
\end{tabular}

Table IV. Possibilities for $G$ and $H_{0}$ in the 6-dimensional case.

Proof. We first show that all the possibilities for $G$ are listed in the table. We know from Proposition 1.19 that $5 \leq \operatorname{dim} G \leq 15$ in this case and $\operatorname{dim} G=\operatorname{dim} H+5$ since $\operatorname{dim}(G / H)=5$. From Section $1.24, G$ must have the form $\left(S^{3}\right)^{m} \times T^{n}$, $\mathrm{SU}(3) \times\left(S^{3}\right)^{m} \times T^{n}, \mathrm{Sp}(2) \times\left(S^{3}\right)^{m} \times T^{n}, \mathrm{G}_{2} \times T^{n}$, or $\operatorname{Spin}(6)$. Further, by Proposition 1.18, we can assume $n \leq 2$ in all cases.

First suppose $G=\left(S^{3}\right)^{m} \times T^{n}$. Then $3 m+n-5=\operatorname{dim} H \leq m$ by Proposition 1.25 and hence $0 \leq 5-2 m-n$. Therefore $m \leq 2$ and if $m=2$ then $n \leq 1$. We see that all of these possibilities are recorded in the table. Next assume $G=\mathrm{SU}(3) \times\left(S^{3}\right)^{m} \times T^{n}$. Then by Proposition 1.25 again we know $8+3 m+n-5=\operatorname{dim} H \leq 4+m$ or $0 \leq 1-2 m-n$. Hence $m=0$ and $n \leq 1$. Note again that these two possibilities for $G$ are listed in the table. Next if $G=\operatorname{Sp}(2) \times\left(S^{3}\right)^{m} \times T^{n}$, Proposition 1.25 gives $0 \leq 1-2 m-n$ again. So again $m=0$ and $n \leq 1$. However, if $G=\operatorname{Sp}(2)$ then $\operatorname{dim} H=5$ and $\operatorname{rank} H \leq \operatorname{rank} G=2$. Yet, from Section 1.24, there are no 5-dimensional compact groups of rank two or less. So in fact, $\operatorname{Sp}(2)$ is not a possibility for $G$. Finally suppose that $G=\mathrm{G}_{2} \times T^{n}$. Then by Proposition 1.25, $\operatorname{dim} H \leq 8$ and yet $H$ would have to be $9+n$-dimensional in this case. Hence this is not a possibility either.

Now we will show that for each $G$ in the table, all the possibilities for $H_{0}$ are listed. First, if $G=G_{1} \times T^{m}$, then for the action to be nonreducible, we can assume $\operatorname{proj}_{2}\left(H_{0}\right)$ is trivial in these cases. Then, we use the list in Section 1.24 to find the possibilities for $H_{0}$ in each case, up to conjugation. For the last case, Proposition 1.20 tells us the full story.

We will now continue with the classification case by case. As in dimension 5, the case that $H$ is discrete is the most difficult.

Case $1_{6}\left(G=S^{3} \times T^{2}\right)$. Here $H$ is discrete. By Proposition 1.18, we see that $K_{0}^{ \pm}$ must both be circle groups in $G$, say $K_{0}^{ \pm}=\left\{\left(e^{x_{ \pm} a_{ \pm} \theta}, e^{i b_{ \pm} \theta}, e^{i c_{ \pm} \theta}\right)\right\}$ for $x_{ \pm} \in \operatorname{Im} S^{3}$, where $\left(b_{-}, c_{-}\right)$and $\left(b_{+}, c_{+}\right)$are linearly independent. After conjugation, we can assume that $x_{-}=i$ and we claim we can also assume that $x_{+}=i$. If one of 
$a_{ \pm}$is zero then this is clear. Otherwise we have $N\left(K_{0}^{-}\right)=\left\{\left(e^{i \theta}, e^{i \phi}, e^{i \psi}\right)\right\}$ and $N\left(K_{0}^{+}\right)=\left\{\left(e^{x_{+} \theta}, e^{i \phi}, e^{i \psi}\right)\right\}$ and $H \subset N\left(K_{0}^{-}\right) \cap N\left(K_{0}^{+}\right)=\left\{\left( \pm 1, e^{i \phi}, e^{i \psi}\right)\right\}$ if $x_{+} \neq$ $\pm i$. But then $H$ would be normal in $G$ and by Proposition 1.2, we would be able to conjugate $K^{+}$to make $x_{+}=i$ without affecting the resulting manifold. So we can assume $K_{0}^{ \pm}=\left\{\left(e^{i a_{ \pm} \theta}, e^{i b_{ \pm} \theta}, e^{i c_{ \pm} \theta}\right)\right\}$.

Let $\alpha_{ \pm}:[0,1] \rightarrow K_{0}^{ \pm}$be curves with $\alpha_{ \pm}(0)=1$ that represent $\pi_{1}\left(K^{ \pm} / H\right)$. Lemma 1.10 says that $M$ will be simply connected if and only if $H$ is generated by $\alpha_{ \pm}(1)$ as a group and the $\alpha_{ \pm}$generate $\pi_{1}(G)$. Assuming that $H$ is generated by the $\alpha_{ \pm}(1)$, we will find the conditions under which the $\alpha_{ \pm}$generate $\pi_{1}(G)$.

Notice that Lemma 1.10 implies that $K^{ \pm}$and $H$ must all be contained in $T^{3}=$ $\left\{\left(e^{i \theta}, e^{i \phi}, e^{i \psi}\right)\right\}$ in order for $M$ to be simply connected. Now consider the cover $\wp: \mathbb{R}^{3} \rightarrow T^{3},(x, y, z) \mapsto\left(e^{2 \pi i x}, e^{2 \pi i y}, e^{2 \pi i z}\right)$. In $\mathbb{R}^{3}$, let $\widetilde{K}^{ \pm}$be the line through the origin and the point $\left(a_{ \pm}, b_{ \pm}, c_{ \pm}\right)$. Then it is clear that $\wp^{-1}\left(K_{0}^{ \pm}\right)=\widetilde{K}^{ \pm}+\mathbb{Z}^{3}$. Next, denote the plane spanned by $\widetilde{K}^{-}$and $\widetilde{K}^{+}$by $Q$ and the lattice $Q \cap \mathbb{Z}^{3}$ by $L$.

We then see that any loop generated by $\alpha_{ \pm}$will lift to a path in $Q$ from the origin to a point in $L=Q \cap \mathbb{Z}^{3}$. Finally define the map $\mathfrak{p}: \mathbb{R}^{3} \rightarrow \mathbb{R}^{2},(x, y, z) \mapsto(y, z)$. Then we have an isomorphism of $\pi_{1}(G) \rightarrow \mathbb{Z}^{2}$ given as follows: For $[c] \in \pi_{1}(G)$ lift $c$ to a curve $\tilde{c}$ in $\mathbb{R}^{3}$ starting from the origin via $\wp$, then take $[c] \mapsto \mathfrak{p}(\tilde{c}(1))$. It is clear that for the combinations of $\alpha_{ \pm}$that form loops in $G$ to generate $\pi_{1}(G)$, we must at least have $\mathfrak{p}(L)=\mathbb{Z}^{2}$. This means that $L$ must have the form

$$
L=\{(f(i, j), i, j) \mid i, j \in \mathbb{Z}\}
$$

for some function $f$ of the form $f(i, j)=r i+s j$ with fixed $r, s \in \mathbb{Z}$. In particular, it follows that $a_{ \pm}=f\left(b_{ \pm}, c_{ \pm}\right)=r b_{ \pm}+s c_{ \pm}$since $\left(a_{ \pm}, b_{ \pm}, c_{ \pm}\right) \in L$. Hence $\operatorname{gcd}\left(b_{ \pm}, c_{ \pm}\right)=1$ since we assumed that $\operatorname{gcd}\left(a_{ \pm}, b_{ \pm}, c_{ \pm}\right)=1$.

Now define the curve $\tilde{\gamma}:[0,1] \rightarrow \mathbb{R}^{3}$ as follows: $\tilde{\gamma}$ starts at the origin, follows $\widetilde{K}^{-}$to the first point of intersection in $\left(\widetilde{K}^{+}+\mathbb{Z}^{3}\right) \cap \widetilde{K}^{-}$, then follows $\widetilde{K}^{+}+\mathbb{Z}^{3}$ to the first integer lattice point $(f(\lambda, \mu), \lambda, \mu)$ in $\mathbb{Z}^{3}$. We now claim that $\left(a_{+}, b_{+}, c_{+}\right)$ and $(f(\lambda, \mu), \lambda, \mu)$ generate $L$. This follows from the fact that there are no points of $L$ in the region of $Q$ strictly between the lines $\widetilde{K}^{+}$and $(f(\lambda, \mu), \lambda, \mu)+\widetilde{K}^{+}$ since there are no lines of $\widetilde{K}^{+}+\mathbb{Z}^{3}$ in this region, by the construction of $\tilde{\gamma}$. Since $\left(a_{+}, b_{+}, c_{+}\right)$and $(f(\lambda, \mu), \lambda, \mu)$ generate $L,\left(b_{+}, c_{+}\right)$and $(\lambda, \mu)$ generate $\mathbb{Z}^{2}$.

Define $\tilde{\delta}_{ \pm}:[0,1] \rightarrow \mathbb{R}^{3}, t \mapsto t\left(a_{ \pm}, b_{ \pm}, c_{ \pm}\right)$and let $\gamma=\wp(\tilde{\gamma})$ and $\delta_{ \pm}=\wp\left(\tilde{\delta}_{ \pm}\right)$. If $d$ denotes the index of $H \cap K_{0}^{-} \cap K_{0}^{+}$in $K_{0}^{-} \cap K_{0}^{+}$, then we claim that $\delta_{+}$and $\gamma^{d}$ generate the same subgroup of $\pi_{1}(G)$ as $\alpha_{-}$and $\alpha_{+}$. To see this, notice that $\alpha_{ \pm}$ can be taken to be paths in $K_{0}^{ \pm}$from the identity to the first element of $H \cap K_{0}^{ \pm}$ and that any combination of $\alpha_{ \pm}$that forms a loop in $G$ can be expressed as a curve in $K_{0}^{-}$, from the identity to an element of $H \cap K_{0}^{-} \cap K_{0}^{+}$, followed by a curve in $K_{0}^{+}$back to the identity. We see from the construction of $\tilde{\gamma}$ that $\gamma^{d}$ is a loop from the identity, along $K_{0}^{-}$to the first element of $H \cap K_{0}^{-} \cap K_{0}^{+}$, then around $K_{0}^{+}$some 
number of times before returning to the identity. Since $H \cap K_{0}^{-} \cap K_{0}^{+} \subset K_{0}^{-} \cap K_{0}^{+}$ are both cyclic subgroups of $K_{0}^{-}$, we see that any loop generated by $\alpha_{-}$and $\alpha_{+}$can be expressed as a power of $\gamma^{d}$ followed by a power of $\delta_{+}$. So $\delta_{+}$and $\gamma^{d}$ generate the same subgroup of $\pi_{1}(G)$ as $\alpha_{-}$and $\alpha_{+}$.

Then by Lemma 1.10, $M$ will be simply connected if and only if $\delta_{+}$and $\gamma^{d}$ generate $\pi_{1}(G)$. Via the isomorphism $\pi_{1}(G) \rightarrow \mathbb{Z}^{2}$ described above, $\delta_{+}$and $\gamma^{d}$ correspond to $\left(b_{+}, c_{+}\right)$and $d(\lambda, \mu)$, respectively. Since $\left(b_{+}, c_{+}\right)$and $(\lambda, \mu)$ generate $\mathbb{Z}^{2}$, from above, it follows that $M$ will be simply connected if and only if $d=1$, or equivalently if $K_{0}^{-} \cap K_{0}^{+} \subset H$.

Hence we have precisely the family $N_{A}^{6}$ of diagrams:

$\left(N_{A}^{6}\right) \quad S^{3} \times T^{2} \supset\left\{\left(e^{i a_{-} \theta}, e^{i b_{-} \theta}, e^{i c_{-} \theta}\right)\right\} \cdot H,\left\{\left(e^{i a_{+} \theta}, e^{i b_{+} \theta}, e^{i c_{+} \theta}\right)\right\} \cdot H \supset H$

$$
\begin{aligned}
\text { where } K^{-} \neq K^{+}, H=H_{-} \cdot H_{+}, \operatorname{gcd}\left(b_{ \pm}, c_{ \pm}\right)=1, \\
\\
a_{ \pm}=r b_{ \pm}+s c_{ \pm}, \text {and } K_{0}^{-} \cap K_{0}^{+} \subset H .
\end{aligned}
$$

We can eliminate several parameters from the expression of the group diagram above. After an automorphism of $G$, we can assume that $K_{0}^{-} \subset S^{3} \times S^{1} \times 1$ and hence $\left(a_{-}, b_{-}, c_{-}\right)=(r, 1,0)$. However the symmetric presentation in $\left(N_{A}^{6}\right)$ will be preferred for our purposes.

We will now address Cases $2{ }_{6}-7_{6}$ from Table IV.

Case $2_{6}\left(G=S^{3} \times S^{3}\right)$. Here $H_{0}=\left\{\left(e^{i p \theta}, e^{i q \theta}\right)\right\}$, for $(p, q)=1$ and $p, q \geq 0$, after conjugation of $G$. Then, from Section 1.24, the possible compact connected proper subgroups $K$ containing $H$ with $K / H \approx S^{l}$ are: any torus $T^{2} \supset H ; S^{3} \times 1$ if $q=0 ; 1 \times S^{3}$ if $p=0 ; \Delta S^{3}$ if $p=q=1 ; S^{3} \times S^{1}$ where $S^{3} \times S^{1} / H \approx S^{3}$ if and only if $q=1$; or $S^{1} \times S^{3}$ where $S^{1} \times S^{3} / H \approx S^{3}$ if and only if $p=1$.

We will now break this into cases by pairing together all of the possibilities for $K^{ \pm}$, remembering that we can switch the places of $K^{-}$and $K^{+}$without affecting the resulting action.

Case $2_{6} \mathrm{~A}\left(K_{0}^{-}\right.$and $K_{0}^{+}$are both tori). Here we need to break this up further into two more cases depending on whether or not $K_{0}^{-}$and $K_{0}^{+}$are the same torus.

Case $2_{6} \mathrm{~A} \mathbf{1}\left(K_{0}^{-}\right.$and $K_{0}^{+}$are the same torus). Here $K_{0}^{-}=K_{0}^{+}=T^{2}$ and hence, by Lemma 1.10, $H \subset K_{0}^{ \pm}$and $K^{ \pm}$are both connected. We also see from Lemma 1.10 that any $H \subset K^{ \pm}$with $H_{0}=S^{1}$ will give a simply connected manifold. In general such groups $H$ will have the form $\left\{\left(e^{i p \theta}, e^{i q \theta}\right)\right\} \cdot \mathbb{Z}_{n}$ after a conjugation of $G$. Therefore we get the family $N_{B}^{6}$ of actions in this case:

$$
S^{3} \times S^{3} \supset\left\{\left(e^{i \theta}, e^{i \phi}\right)\right\},\left\{\left(e^{i \theta}, e^{i \phi}\right)\right\} \supset\left\{\left(e^{i p \theta}, e^{i q \theta}\right)\right\} \cdot \mathbb{Z}_{n}
$$

Case $2{ }_{6} \mathrm{A2}\left(K_{0}^{-}\right.$and $K_{0}^{+}$are different tori). For $K_{0}^{-}$and $K_{0}^{+}$to be different tori, both containing the circle $H_{0}=\left\{\left(e^{i p \theta}, e^{i q \theta}\right)\right\}$, it follows that either $p$ or $q$ must be 
zero. Suppose, without loss of generality that $q=0$, so that $H_{0}=\left\{\left(e^{i \theta}, 1\right)\right\}$. It then follows that $K_{0}^{ \pm}$must have the form $K_{0}^{ \pm}=\left\{\left(e^{i \theta}, e^{x_{ \pm} \phi}\right)\right\}$ for some $x_{ \pm} \in \operatorname{Im}\left(S^{3}\right)$. For $M$ to be simply connected $H \subset\left\{\left(e^{i \theta}, g\right)\right\}=S^{1} \times S^{3}$ by Lemma 1.10 . Therefore $H$ and $K^{ \pm}$all have the form $H=S^{1} \times \widehat{H}$ and $K^{ \pm}=S^{1} \times \widehat{K}^{ \pm}$, where $\widehat{K}^{ \pm} / \widehat{H} \approx S^{1}$. That means $S^{3} \supset \widehat{K}^{+}, \widehat{K}^{-} \supset \widehat{H}$ gives a four-dimensional cohomogeneity one manifold. Further, from Lemma 1.10, it follows that this 4-manifold will be simply connected if and only if $M$ is. Hence our action is a product action with some simply connected 4-manifold.

Case $2_{6} \mathbf{B}\left(K_{0}^{-}=T^{2}\right.$ and $\left.K_{0}^{+}=S^{3} \times 1\right)$. Then $q=0$ and $H_{0}=\left\{\left(e^{i \theta}, 1\right)\right\}$.

From Lemma 1.10, $H$ must be of the form $S^{1} \times \mathbb{Z}_{n} \subset T^{2}$. This gives the family $N_{C}^{6}$ of group diagrams:

$$
S^{3} \times S^{3} \supset T^{2}, S^{3} \times \mathbb{Z}_{n} \supset S^{1} \times \mathbb{Z}_{n}
$$

Conversely, these diagrams obviously determine simply connected manifolds, by Lemma 1.10 .

Case $2_{6} \mathbf{C}\left(K_{0}^{-}=T^{2}\right.$ and $\left.K_{0}^{+}=\Delta S^{3}\right)$. Then $p=q=1$ and $H_{0}=\Delta S^{1}$.

Again, by Lemma $1.10, H$ will have the form $\Delta S^{1} \cdot \mathbb{Z}_{n}$. Yet, every compact Lie group is contained in the normalizer of its identity component. In particular $K^{+} \subset N\left(\Delta S^{3}\right)= \pm \Delta S^{3}=\{(g, \pm g)\}$. This means that $n$ is at most two. Therefore, we have the following two possibilities for group diagrams:

$$
S^{3} \times S^{3} \supset T^{2}, \Delta S^{3} \cdot \mathbb{Z}_{n} \supset \Delta S^{1} \cdot \mathbb{Z}_{n}, \quad \text { where } n=1 \text { or } 2 .
$$

From Lemma 1.10, we see that these are both in fact simply connected.

Case $2_{6} \mathbf{D}\left(K_{0}^{-}=T^{2}\right.$ and $\left.K_{0}^{+}=S^{3} \times S^{1}\right)$. Then $q=1$ and $H_{0}=\left\{\left(e^{i p \theta}, e^{i \theta}\right)\right\}$.

It is clear in this case that $K_{0}^{-} \subset K_{0}^{+}$. Further, for $K^{+} / H$ to be a 3-sphere, $H \cap K_{0}^{+}=H_{0}$. Therefore $H$ and $K^{ \pm}$are all connected. We then have the family $N_{D}^{6}$ of diagrams, which all give simply connected manifolds by Lemma 1.10:

$$
S^{3} \times S^{3} \supset T^{2}, S^{3} \times S^{1} \supset\left\{\left(e^{i p \theta}, e^{i \theta}\right)\right\} .
$$

Case $2_{6} \mathbf{E}\left(K_{0}^{-}=S^{3} \times 1\right.$ and $\left.K_{0}^{+}=S^{3} \times 1\right)$. Then $q=0$ and $H_{0}=\left\{\left(e^{i \theta}, 1\right)\right\}$.

From Corollary 1.9, we know that $H$ and $K^{ \pm}$must all be connected in this case. We then have the following group diagram, which gives a simply connected manifold by Lemma 1.10:

$$
S^{3} \times S^{3} \supset S^{3} \times 1, S^{3} \times 1 \supset S^{1} \times 1 .
$$

We note that this is a product action. 
Case $2{ }_{6} \mathbf{F}\left(K_{0}^{-}=S^{3} \times 1\right.$ and $\left.K_{0}^{+}=S^{1} \times S^{3}\right)$. Then $q=0, p=1$ and $H_{0}=\left\{\left(e^{i \theta}, 1\right)\right\}$. As in the previous case, we get the following simply connected group diagram:

$$
S^{3} \times S^{3} \supset S^{3} \times 1, S^{1} \times S^{3} \supset S^{1} \times 1 .
$$

We see that this is a sum action.

Case $2{ }_{6} \mathbf{G}\left(K_{0}^{-}=\Delta S^{3}\right.$ and $\left.K_{0}^{+}=\Delta S^{3}\right)$. Then $p=q=1$ and $H_{0}=\Delta S^{1}$.

As above we have the following group diagram:

$$
S^{3} \times S^{3} \supset \Delta S^{3}, \Delta S^{3} \supset \Delta S^{1} .
$$

Case ${ }_{6} \mathbf{H}\left(K_{0}^{-}=\Delta S^{3}\right.$ and $\left.K_{0}^{+}=S^{3} \times S^{1}\right)$. Then $p=q=1$ and $H_{0}=\Delta S^{1}$.

Again, we have

$$
S^{3} \times S^{3} \supset \Delta S^{3}, S^{3} \times S^{1} \supset \Delta S^{1} .
$$

Case $2_{6} \mathbf{I}\left(K_{0}^{-}=S^{3} \times S^{1}\right.$ and $\left.K_{0}^{+}=S^{3} \times S^{1}\right)$. Then $q=1$ and $H_{0}=\left\{\left(e^{i p \theta}, e^{i \theta}\right)\right\}$. Here, as above, we have the family $N_{E}^{6}$ :

$$
S^{3} \times S^{3} \supset S^{3} \times S^{1}, S^{3} \times S^{1} \supset\left\{\left(e^{i p \theta}, e^{i \theta}\right)\right\} .
$$

Case $2_{6} \mathbf{J}\left(K_{0}^{-}=S^{3} \times S^{1}\right.$ and $\left.K_{0}^{+}=S^{1} \times S^{3}\right)$. Then $p=q=1$ and $H_{0}=\Delta S^{1}$.

Our last possibility in this case is the following diagram:

$$
S^{3} \times S^{3} \supset S^{3} \times S^{1}, S^{1} \times S^{3} \supset \Delta S^{1} .
$$

Case $3_{6}\left(G=S^{3} \times S^{3} \times S^{1}\right)$. Here $H_{0}=T^{2} \times 1 \subset S^{3} \times S^{3} \times S^{1}$. By Proposition 1.18, one of $K^{ \pm} / H$ must be a circle, say $K^{-} / H \approx S^{1}$. Furthermore, since $\operatorname{rank}(H)=$ $\operatorname{rank}\left(S^{3} \times S^{3}\right)$, Proposition 1.18 says $K^{-}=T^{2} \times S^{1}$, and all of $H, K^{-}$and $K^{+}$ are connected. We will now find the possibilities for $K^{+}$. Notice that if $\operatorname{proj}_{3}\left(K^{+}\right)$ is nontrivial, then by Proposition $1.18, K^{+}=T^{2} \times S^{1}$, giving one possibility. Otherwise $K^{+} \subset S^{3} \times S^{3} \times 1$. In this case, $K^{+}$, which must contain $H$, must be one of $S^{3} \times S^{1} \times 1, S^{1} \times S^{3} \times 1$ or $S^{3} \times S^{3} \times 1$. But $S^{3} \times S^{3} \times 1 / S^{1} \times S^{1} \times 1 \approx S^{2} \times S^{2}$, which is not a sphere. Putting this together, we see our only possible group diagrams, up to automorphism, are

$$
\begin{aligned}
& S^{3} \times S^{3} \times S^{1} \supset S^{1} \times S^{1} \times S^{1}, S^{1} \times S^{1} \times S^{1} \supset S^{1} \times S^{1} \times 1, \\
& S^{3} \times S^{3} \times S^{1} \supset S^{1} \times S^{1} \times S^{1}, S^{3} \times S^{1} \times 1 \supset S^{1} \times S^{1} \times 1 .
\end{aligned}
$$

We see, however, that both of these actions are product actions.

Case $4_{6}(G=\mathrm{SU}(3))$. Here $H_{0}$ must be $\mathrm{SO}(3)$ or $\mathrm{SU}(2)$. Since $\mathrm{SO}(3)$ is maximalconnected in $\mathrm{SU}(3)$, we may disregard this case by Proposition 1.22. So assume $H_{0}=\mathrm{SU}(2)=\{\operatorname{diag}(A, 1)\}$.

Then a proper closed subgroup $K$ with $K / H \approx S^{l}$ must be a conjugate of $\mathrm{U}(2)$, from Section 1.24. Now notice that the only conjugate of $U(2)$ that contains 
$\mathrm{SU}(2)=\{\operatorname{diag}(A, 1)\}$ is $\mathrm{U}(2)=\{\operatorname{diag}(A, \operatorname{det}(\bar{A}))\}$. So we can assume $K_{0}^{ \pm}=$ $\mathrm{U}(2)=\{\operatorname{diag}(A, \operatorname{det}(\bar{A}))\}$.

Recall that $H$ must be generated by a subgroup of $K_{0}^{ \pm}=\{\operatorname{diag}(A, \operatorname{det}(\bar{A}))\}$. Therefore $H=H_{0} \cdot \mathbb{Z}_{n} \subset K_{0}^{ \pm}$and $K^{ \pm}$are connected. We then get the family $N_{F}^{6}$ of diagrams:

$\left(N_{F}^{6}\right) \quad \operatorname{SU}(3) \supset\{\operatorname{diag}(A, \operatorname{det}(\bar{A}))\},\{\operatorname{diag}(A, \operatorname{det}(\bar{A}))\} \supset\{\operatorname{diag}(A, 1)\} \cdot \mathbb{Z}_{n}$

Conversely these all give simply connected manifolds by Lemma 1.10.

Case $5_{6}\left(G=\operatorname{SU}(3) \times S^{1}\right)$. Now $H_{0}=\mathrm{U}(2) \times 1=\{\operatorname{diag}(A, \operatorname{det}(\bar{A}))\} \times 1$. Since $\operatorname{rank}(\mathrm{U}(2))=\operatorname{rank}(\mathrm{SU}(3))$ we may assume $K^{-}=\mathrm{U}(2) \times S^{1}$ by Proposition 1.18, and that $H$ and $K^{+}$are connected. Then from Section 1.24, $\operatorname{proj}_{1}\left(K^{+}\right)$is either $\mathrm{U}(2)$ or $\mathrm{SU}(3)$. However $\mathrm{SU}(3) / \mathrm{U}(2)$ is not a sphere so $K^{+}=\mathrm{U}(2) \times S^{1}$ by Proposition 1.18. Therefore we have the following possibility:

$$
\mathrm{SU}(3) \times S^{1} \supset \mathrm{U}(2) \times S^{1}, \mathrm{U}(2) \times S^{1} \supset \mathrm{U}(2) \times 1
$$

which we see is simply connected by Lemma 1.10 . However, we see this is a product action.

Case $6_{6}\left(G=\operatorname{Sp}(2) \times S^{1}\right)$. Here $H_{0}=\operatorname{Sp}(1) \operatorname{Sp}(1) \times 1$. To find the possibilities for connected groups $K$ with $K / H \approx S^{l}$, note that if $\operatorname{proj}_{2}(K) \subset S^{1}$ is nontrivial then $K=\operatorname{Sp}(1) \operatorname{Sp}(1) \times S^{1}$, by Proposition 1.18. Otherwise $K \subset \mathrm{Sp}(2) \times 1$ and hence from Section $1.24, K=\operatorname{Sp}(2) \times 1$. In either case $K / H \approx S^{l}$, in fact. Further by Proposition 1.18, we can assume $K^{-}=\operatorname{Sp}(1) \operatorname{Sp}(1) \times S^{1}$ and all of $K^{ \pm}$and $H$ are connected. Therefore we have the two possibilities

$$
\begin{aligned}
& \mathrm{Sp}(2) \times S^{1} \supset \mathrm{Sp}(1) \operatorname{Sp}(1) \times S^{1}, \operatorname{Sp}(1) \operatorname{Sp}(1) \times S^{1} \supset \operatorname{Sp}(1) \operatorname{Sp}(1) \times 1, \\
& \mathrm{Sp}(2) \times S^{1} \supset \mathrm{Sp}(1) \operatorname{Sp}(1) \times S^{1}, \operatorname{Sp}(2) \times 1 \supset \operatorname{Sp}(1) \operatorname{Sp}(1) \times 1,
\end{aligned}
$$

both of which are simply connected by Lemma 1.10 . We easily see that the first action is a product action and the second action is a sum action.

Case $7_{6}(G=\operatorname{Spin}(6))$. In this case we know from Proposition 1.20 that this gives a two-fixed-point action on a sphere.

\section{Classification in dimension seven}

As in the previous section we keep the notation and conventions established in Section 2, this time for a 7-dimensional manifold $M$. In this case, the next proposition gives us the possibilities for $G$ and $H_{0}$.

Proposition 4.1. Table V lists all the possibilities for $G$ and $H_{0}$, up to equivalence. 


\begin{tabular}{ccc}
\hline Case & $G$ & $H_{0}$ \\
\hline $1_{7}$ & $S^{3} \times S^{3}$ & $\{1\}$ \\
$2_{7}$ & $S^{3} \times S^{3} \times S^{1}$ & $\left\{\left(e^{i p \theta}, e^{i q \theta}\right)\right\} \times 1$ \\
$3_{7}$ & $S^{3} \times S^{3} \times T^{2}$ & $T^{2} \times 1$ \\
$4_{7}$ & $\mathrm{SU}(3)$ & $T^{2}$ \\
$5_{7}$ & $S^{3} \times S^{3} \times S^{3}$ & $T^{3}$ \\
$6_{7}$ & $\mathrm{SU}(3) \times S^{1}$ & $\mathrm{SU}(2) \times 1, \mathrm{SO}(3) \times 1$ \\
$7_{7}$ & $\mathrm{SU}(3) \times T^{2}$ & $\mathrm{U}(2) \times 1$ \\
$8_{7}$ & $\mathrm{Sp}(2)$ & $\mathrm{U}(2)_{\max }, \mathrm{Sp}(1) \mathrm{SO}(2)$ \\
$9_{7}$ & $\mathrm{SU}(3) \times S^{3}$ & $\mathrm{U}(2) \times S^{1}$ \\
$10_{7}$ & $\mathrm{Sp}(2) \times T^{2}$ & $\mathrm{Sp}(1) \mathrm{Sp}(1) \times 1$ \\
$11_{7}$ & $\mathrm{Sp}(2) \times S^{3}$ & $\mathrm{Sp}(1) \mathrm{Sp}(1) \times S^{1}$ \\
$12_{7}$ & $\mathrm{G}$ & $\mathrm{SU}(3)$ \\
$13_{7}$ & $\mathrm{SU}(4)$ & $\mathrm{U}(3)$ \\
$14_{7}$ & $\mathrm{SU}(4) \times S^{1}$ & $\mathrm{Sp}(2) \times 1$ \\
$15_{7}$ & $\mathrm{Spin}(7)$ & $\mathrm{Spin}(6)$ \\
\hline
\end{tabular}

Table V. Possibilities for $G$ and $H_{0}$ in the 7-dimensional case.

Proof. We will first show that all the possibilities for $G$ are listed in Table V. Recall that $6 \leq \operatorname{dim}(G) \leq 21$ by Proposition 1.19 and that $\operatorname{dim} H=\operatorname{dim} G-6$, since $\operatorname{dim} G / H=\operatorname{dim} M-1=6$ in this case. A priori, from Section 1.24, we need to check all of the possibilities for $G$ of the form $\left(S^{3}\right)^{m} \times T^{n},(\operatorname{SU}(3))^{l} \times\left(S^{3}\right)^{m} \times T^{n}$, $(\mathrm{Sp}(2))^{k} \times\left(S^{3}\right)^{m} \times T^{n}, \mathrm{G}_{2} \times\left(S^{3}\right)^{m} \times T^{n}, \mathrm{SU}(4) \times\left(S^{3}\right)^{m} \times T^{n}, \mathrm{Sp}(2) \times \mathrm{SU}(3) \times$ $\left(S^{3}\right)^{m} \times T^{n}, \operatorname{Sp}(3)$ and $\operatorname{Spin}(7)$. Note that by Proposition 1.18 we can assume that $n \leq 2$ in all cases.

First suppose $G=\left(S^{3}\right)^{m} \times T^{n}$. By Proposition 1.25, 3m+n-6=dim $(H) \leq m$, which means $0 \leq 6-2 m-n$ and so $m \leq 3$ and if $m=3$ then $n=0$. Notice that all of these possibilities are listed in the table. Next if $G=(\mathrm{SU}(3))^{l} \times\left(S^{3}\right)^{m} \times T^{n}$ for $l>0$, then as before $8 l+3 m+n-6=\operatorname{dim}(H) \leq 4 l+m$ or $0 \leq 6-4 l-2 m-n$. Hence $l=1$, and $m=1$ and $n=0$, or $m=0$ and $n \leq 2$. All of these possibilities are listed in the table. Next suppose $G=(\operatorname{Sp}(2))^{k} \times\left(S^{3}\right)^{m} \times T^{n}$. Then we get $10 k+3 m+n-6=\operatorname{dim}(H) \leq 6 k+m$ or $0 \leq 6-4 k-2 m-n$. As before $k=1$, and $m=1$ and $n=0$, or $m=0$ and $n \leq 2$. However, if $G=\operatorname{Sp}(2) \times S^{1}$ then $\operatorname{dim} H=5$ and by Proposition 1.18, $H_{0} \subset \mathrm{Sp}(2) \times 1$. Then $\operatorname{rank} H \leq \operatorname{rank} \operatorname{Sp}(2)=2$ and yet there are no compact 5-dimensional groups of rank 2 or less. So $\operatorname{Sp}(2) \times S^{1}$ is not a possibility for $G$. Next, if $G=\operatorname{Sp}(2) \times \mathrm{SU}(3) \times\left(S^{3}\right)^{m} \times T^{n}$ then $0 \leq-2-2 m-n$, which is impossible. Now say $G=\mathrm{G}_{2} \times\left(S^{3}\right)^{m} \times T^{n}$. We get $14+3 m+n-6 \leq 8+m$ or $0 \leq-2 m-n$ and hence $m=n=0$. Lastly, if $G=\mathrm{SU}(4) \times\left(S^{3}\right)^{m} \times T^{n}$ then $15+3 m+n-6 \leq 10+m$ or $0 \leq 1-2 m-n$. Therefore $m=0$ and $n \leq 1$. 
Finally if $\operatorname{dim}(G)=21$ we know from Proposition 1.20 that $G$ must be isomorphic to $\operatorname{Spin}(7)$ and in this case $H$ will be $\operatorname{Spin}(6)$.

Next we check that in the rest of the cases, we have listed all the possibilities for $H_{0}$. Again, we can assume that $H_{0} \subset G_{1} \times 1$ in the cases that $G=G_{1} \times T^{m}$. Then we use Section 1.24 to find the possibilities for $H_{0}$. The only exceptional cases are $9_{7}$ and $11_{7}$, where $G=G_{1} \times S^{3}$. By Proposition $1.25, H_{0} \subset L \times S^{1}$, where $L$ is of dimension 4 or less in Case $9_{7}$ and dimension 6 or less in Case $11_{7}$. However, since $\operatorname{dim} H=\operatorname{dim} G-6$, we see that $H_{0}=L \times S^{1}$ where $L$ is of maximal dimension in each case. From Section 1.24, we see that $H_{0}$ must be one of the groups listed below.

As in the previous sections we find all possible diagrams case by case.

Cases $2_{7}$ and $6_{7}$. Both cases involve the same difficulty that arises in the case of $G=S^{3} \times S^{1}$ in dimension 5 .

Lemma 4.2. Let $M$ be a simply connected cohomogeneity one manifold given by the group diagram $G \supset K^{-}, K^{+} \supset H$, with $G=G_{1} \times S^{1}$ for $G_{1}$ simply connected and $H_{0}=H_{1} \times 1$. Suppose further that there is a compact subgroup $L \subset G_{1}$ of the form $L=H_{1} \cdot\{\beta(\theta)\}$, where $\{\beta(\theta)\}$ is a circle group of $G$ parameterized once around by $\beta:[0,1] \rightarrow G_{1}$ and $\{\beta(\theta)\} \cap H_{1}=1$. Define $\delta:[0,1] \rightarrow G, t \mapsto\left(1, e^{2 \pi i t}\right)$ to be a loop once around $1 \times S^{1}$. If $K_{0}^{ \pm} \subset L \times S^{1}$, then the group diagram for $M$ has one of the following forms, all of which give simply connected manifolds:

$G_{1} \times S^{1} \supset H_{+} \cdot\left\{\left(\beta\left(m_{-} \theta\right), \delta\left(n_{-} \theta\right)\right)\right\}, H_{-} \cdot\left\{\left(\beta\left(m_{+} \theta\right), \delta\left(n_{+} \theta\right)\right)\right\} \supset H$, where $H=H_{-} \cdot H_{+}, K^{-} \neq K^{+}, \operatorname{gcd}\left(n_{-}, n_{+}, d\right)=1$ and $d$ is the index of $H \cap K_{0}^{-} \cap K_{0}^{+}$in $K_{0}^{-} \cap K_{0}^{+}$, $G_{1} \times S^{1} \supset\{(\beta(m \theta), \delta(\theta))\} \cdot H_{0},\{(\beta(m \theta), \delta(\theta))\} \cdot H_{0} \supset H_{0} \cdot \mathbb{Z}_{n}$,

where $\mathbb{Z}_{n} \subset\{(\beta(m \theta), \delta(\theta))\}$.

Proof. It is clear, as in Proposition 1.18 , that $K^{ \pm} / H$ must be circles and hence $K_{0}^{ \pm}=H_{0} \cdot\left\{\left(\beta\left(m_{ \pm} \theta\right), \delta\left(n_{ \pm} \theta\right)\right)\right\}$. From Lemma 1.10, $H$ must have the form $H=$ $H_{-} \cdot H_{+}$for $H_{ \pm}=K_{0}^{ \pm} \cap H=H_{0} \cdot \mathbb{Z}_{k_{ \pm}}$, with $\mathbb{Z}_{k_{ \pm}} \subset\left\{\left(\beta\left(m_{ \pm} \theta\right), \delta\left(n_{ \pm} \theta\right)\right)\right\}$. Then with the notation of Lemma 1.10, we see that the $\alpha_{ \pm}$can be taken as $\alpha_{ \pm}(t)=$ $\left(\beta\left(m_{ \pm} t / k_{ \pm}\right), \delta\left(n_{ \pm} t / k_{ \pm}\right)\right)$. Then Lemma 1.10 says that $M$ is simply connected if and only if the $\alpha_{ \pm}$generate $\pi_{1}\left(G / H_{0}\right)$. Since $\{\beta(\theta)\} \cap H_{1}=1$ we see that $\{\beta(\theta)\}$ injects onto a circle in $G / H_{0}$ that is contractible since $G_{1}$ is simply connected. We also see that $\delta$ generates $\pi_{1}\left(G / H_{0}\right)$ since $H_{0} \subset G_{1} \times 1$.

This brings us precisely to the situation we encountered in Case $1{ }_{5} \mathrm{~B} 2$. The argument there shows that if $K_{0}^{-}=K_{0}^{+}$we get the second diagram from the lemma, and if $K_{0}^{-} \neq K_{0}^{+}$, then $M$ is simply connected if and only if $\operatorname{gcd}\left(n_{-}, n_{+}, d\right)=1$, 
where $d$ is the index of $H / H_{0} \cap K_{0}^{-} / H_{0} \cap K_{0}^{+} / H_{0}$ in $K_{0}^{-} / H_{0} \cap K_{0}^{+} / H_{0}$. We can also write $d$ as the index of $H \cap K_{0}^{-} \cap K_{0}^{+}$in $K_{0}^{-} \cap K_{0}^{+}$.

We now address Cases 27 and $6_{7}$ individually, using the lemma when needed.

Case $2_{7}\left(G=S^{3} \times S^{3} \times S^{1}\right)$. Here $H_{0}=\left\{\left(e^{i p \theta}, e^{i q \theta}, 1\right)\right\}$. After an automorphism of $G$ we can assume that $p \geq q \geq 0$ and in particular $p \neq 0$. We know from Proposition 1.18 that $K_{0}^{-}$, say, is a two torus. After conjugation we can assume that $K_{0}^{-}=\left\{\left(e^{i a_{-} \theta}, e^{i b_{-} \theta}, e^{i c_{-} \theta}\right)\right\} \cdot\left\{\left(e^{i p \theta}, e^{i q \theta}, 1\right)\right\}$ even if $q=0$. From Proposition 1.18, if $\operatorname{proj}_{3}\left(K_{0}^{+}\right)$is nontrivial then $K_{0}^{+}$is also a torus. Otherwise $K_{0}^{+} \subset S^{3} \times S^{3} \times 1$. Therefore from Section 1.24, we see that $K_{0}^{+}$must be one of the following groups: $T^{2}, S^{3} \times 1 \times 1$ if $q=0, \Delta S^{3} \times 1$ if $p=q=1$, or $S^{3} \times S^{1} \times 1$ if $q=1$ and allowing arbitrary $p$. We will now break this up into cases depending on what $K_{0}^{+}$is.

Case $2{ }_{7} \mathrm{~A}\left(\operatorname{dim} K^{+}>2\right)$. Then by Corollary $1.9, K^{-}$is connected and $M$ is simply connected if and only if $G / K^{-}$is. So we can assume $K^{-}=\left\{\left(e^{i a \theta}, e^{i b \theta}, e^{i \theta}\right)\right\}$. $\left\{\left(e^{i p \theta}, e^{i q \theta}, 1\right)\right\}$, that is, $c=1$. We also know that $H$ is a subgroup of $K^{-}$of the form $\left\{\left(e^{i p \theta}, e^{i q \theta}, 1\right)\right\} \cdot \mathbb{Z}_{n}$ for $\mathbb{Z}_{n} \subset\left\{\left(e^{i a \theta}, e^{i b \theta}, e^{i \theta}\right)\right\}$ such that $\mathbb{Z}_{n} \cap K_{0}^{+}=1$, which is automatic, and $\mathbb{Z}_{n} \subset N\left(K_{0}^{+}\right)$.

Case $27 \mathbf{A 1}\left(K_{0}^{+}=S^{3} \times 1 \times 1\right.$ and $\left.q=0\right)$. Then $H_{0}=S^{1} \times 1 \times 1$ and $K^{-}=$ $\left\{\left(1, e^{i b \theta}, e^{i \theta}\right)\right\} \cdot\left\{\left(e^{i \theta}, 1,1\right)\right\}$. Then we see that the $\mathbb{Z}_{n}$ in $H$ can be arbitrary and we get the family:

$\left(Q_{C}^{7}\right) \quad S^{3} \times S^{3} \times S^{1} \supset\left\{\left(e^{i \phi}, e^{i b \theta}, e^{i \theta}\right)\right\}, S^{3} \times 1 \times 1 \cdot \mathbb{Z}_{n} \supset S^{1} \times 1 \times 1 \cdot \mathbb{Z}_{n}$, where $\mathbb{Z}_{n} \subset\left\{\left(1, e^{i b \theta}, e^{i \theta}\right)\right\}$.

Case $2{ }_{7} \mathbf{A 2}\left(K_{0}^{+}=\Delta S^{3} \times 1\right.$ and $\left.p=q=1\right)$. Here $H_{0}=\left\{\left(e^{i \theta}, e^{i \theta}, 1\right)\right\}$ and we can take $K^{-}=\left\{\left(1, e^{i b \theta}, e^{i \theta}\right)\right\} \cdot\left\{\left(e^{i \theta}, e^{i \theta}, 1\right)\right\}$ for a new $b$. Then for $\mathbb{Z}_{n} \subset\left\{\left(1, e^{i b \theta}, e^{i \theta}\right)\right\}$ to satisfy $\mathbb{Z}_{n} \subset N\left(K_{0}^{+}\right)$simply means that $n \mid 2 b$. Then the further condition that $H \cap 1 \times 1 \times S^{1}=1$ for the action to be effective means that $n$ is 1 or 2 . Therefore we have these diagrams in this case:

$\left(Q_{D}^{7}\right) \quad S^{3} \times S^{3} \times S^{1} \supset\left\{\left(e^{i \phi}, e^{i \phi} e^{i b \theta}, e^{i \theta}\right)\right\}, \Delta S^{3} \times 1 \cdot \mathbb{Z}_{n} \supset \Delta S^{1} \times 1 \cdot \mathbb{Z}_{n}$, with $\mathbb{Z}_{n} \subset\left\{\left(1, e^{i b \theta}, e^{i \theta}\right)\right\}$, where $n$ is 1 or 2 .

This family will be described in more detail in Section 5.2.

Case $2_{7} \mathbf{A 3}\left(K_{0}^{+}=S^{3} \times S^{1} \times 1, q=1\right.$ and $p$ arbitrary $)$. Here $H_{0}=\left\{\left(e^{i p \theta}, e^{i \theta}, 1\right)\right\}$ and we can take $K^{-}=\left\{\left(e^{i a \theta}, 1, e^{i \theta}\right)\right\} \cdot\left\{\left(e^{i p \theta}, e^{i \theta}, 1\right)\right\}$ for a new $a$. Then the $\mathbb{Z}_{n} \subset\left\{\left(e^{i a \theta}, 1, e^{i \theta}\right)\right\}$ in $H$ automatically satisfies the condition $\mathbb{Z}_{n} \subset N\left(K_{0}^{+}\right)$. Hence we have the diagrams

$$
\begin{array}{r}
\left(N_{F}^{7}\right) \quad S^{3} \times S^{3} \times S^{1} \supset\left\{\left(e^{i p \phi} e^{i a \theta}, e^{i \phi}, e^{i \theta}\right)\right\}, S^{3} \times S^{1} \times \mathbb{Z}_{n} \supset\left\{\left(e^{i p \phi}, e^{i \phi}, 1\right)\right\} \cdot \mathbb{Z}_{n} \\
\mathbb{Z}_{n} \subset\left\{\left(e^{i a \theta}, 1, e^{i \theta}\right)\right\} .
\end{array}
$$


Case $2{ }_{7} \mathbf{B}\left(\operatorname{dim} K^{+}=2\right.$ so $\left.K_{0}^{+} \approx T^{2}\right)$. Here $H_{0}=\left\{\left(e^{i p \theta}, e^{i q \theta}, 1\right)\right\}$, again where we assume $p \geq q \geq 0$ and $K_{0}^{-}=\left\{\left(e^{i a_{-} \theta}, e^{i b_{-} \theta}, e^{i c_{-} \theta}\right)\right\} \cdot\left\{\left(e^{i p \theta}, e^{i q \theta}, 1\right)\right\}$. We now break this into two cases depending on whether or not $q$ is zero.

Case $2{ }_{7} \mathbf{B 1}(q=0)$. Here $H_{0}=S^{1} \times 1 \times 1$ and so we know that $K_{0}^{ \pm}=S^{1} \times \bar{K}_{0}^{ \pm}$for some groups $\bar{K}_{0}^{ \pm} \subset S^{3} \times S^{1}$. Then from Lemma 1.10, $H$ has the form $S^{1} \times \bar{H}$ for a subgroup $\bar{H}$ generated by $\bar{H} \cap \bar{K}_{0}^{-}$and $\bar{H} \cap \bar{K}_{0}^{+}$. Similarly, by Lemma 1.10 , the manifold $M$ will be simply connected if and only if the 5-manifold $\bar{M}$ given by the group diagram $S^{3} \times S^{1} \supset \bar{K}^{-}, \bar{K}^{+} \supset \bar{H}$ is simply connected. So these actions are product actions with some simply connected 5-dimensional cohomogeneity one manifold.

Case $2{ }_{7} \mathbf{B 2}(p, q \neq 0)$. We can take $K_{0}^{ \pm}=\left\{\left(e^{i a_{ \pm} \theta}, e^{i b_{ \pm} \theta}, e^{i c_{ \pm} \theta}\right)\right\} \cdot\left\{\left(e^{i p \theta}, e^{i q \theta}, 1\right)\right\}$ although there is a more convenient way to write these groups in our case. Note that for $p \mu-q \lambda=1$, we can write any element of the torus $T^{2}$ uniquely as $\left(e^{i p \theta}, e^{i q \theta}\right)\left(e^{i \lambda \phi}, e^{i \mu \phi}\right)=\left(z^{p}, z^{q}\right)\left(w^{\lambda}, w^{\mu}\right)$. Then we can write

$$
K_{0}^{ \pm}=\left\{\left(z^{p}, z^{q}, 1\right)\left(w^{m_{ \pm} \lambda}, w^{m_{ \pm} \mu}, w^{n_{ \pm}}\right)\right\}
$$

for some $m_{ \pm}, n_{ \pm} \in \mathbb{Z}$ with $\operatorname{gcd}\left(m_{ \pm}, n_{ \pm}\right)=1$. Then letting $\beta(t)=\left(e^{2 \pi i \lambda t}, e^{2 \pi i \mu t}\right)$, we see this satisfies the conditions of Lemma 4.2. By that lemma, we have precisely these two families of diagrams:

$$
\begin{array}{r}
S^{3} \times S^{3} \times S^{1} \supset\left\{\left(z^{p} w^{\lambda m_{-}}, z^{q} w^{\mu m_{-}}, w^{n_{-}}\right)\right\} H,\left\{\left(z^{p} w^{\lambda m_{+}}, z^{q} w^{\mu m_{+}}, w^{n_{+}}\right)\right\} H \supset H, \\
\text { where } H=H_{-} \cdot H_{+}, H_{0}=\left\{\left(z^{p}, z^{q}, 1\right)\right\}, K^{-} \neq K^{+}, p \mu-q \lambda=1, \\
\operatorname{gcd}\left(n_{-}, n_{+}, d\right)=1, \text { where } d \text { is the index of } H \cap K_{0}^{-} \cap K_{0}^{+} \text {in } K_{0}^{-} \cap K_{0}^{+}, \\
S^{3} \times S^{3} \times S^{1} \supset\left\{\left(z^{p} w^{\lambda m}, z^{q} w^{\mu m}, w\right)\right\},\left\{\left(z^{p} w^{\lambda m}, z^{q} w^{\mu m}, w\right)\right\} \supset H_{0} \cdot \mathbb{Z}_{n}, \\
\text { where } H_{0}=\left\{\left(z^{p}, z^{q}, 1\right)\right\}, p \mu-q \lambda=1 \text { and } \mathbb{Z}_{n} \subset\left\{\left(w^{\lambda m}, w^{\mu m}, w\right)\right\} .
\end{array}
$$

These two families are $N_{E}^{7}$ and $N_{D}^{7}$, respectively.

Case $6_{7}\left(G=\mathrm{SU}(3) \times S^{1}\right)$. Here $H_{0}$ is either $\mathrm{SU}(2) \times 1$ or $\mathrm{SO}(3) \times 1$. First, if $H_{0}=$ $\mathrm{SO}(3) \times 1$ then $H_{1}=\mathrm{SO}(3)$ is maximal in $\mathrm{SU}(3)$ and so by Proposition $1.18, H, K^{-}$ and $K^{+}$are all connected, $K^{-}$, say, is $\mathrm{SO}(3) \times S^{1}$ and $K^{+}$is either $\mathrm{SO}(3) \times S^{1}$ or $\mathrm{SU}(3) \times 1$. Since $\mathrm{SU}(3) / \mathrm{SO}(3)$ is not a sphere we have only one possible diagram,

$$
\mathrm{SU}(3) \times S^{1} \supset \mathrm{SO}(3) \times S^{1}, \mathrm{SO}(3) \times S^{1} \supset \mathrm{SO}(3) \times 1,
$$

which comes from a product action.

For the other case assume $H_{0}=\mathrm{SU}(2) \times 1$ where $\mathrm{SU}(2)=\mathrm{SU}(1) \mathrm{SU}(2)$ is the lower right block. From Section 1.24, $\operatorname{proj}_{1}\left(K_{0}^{ \pm}\right)$is either $\mathrm{SU}(2), \mathrm{U}(2)$ or SU(3). As in Proposition 1.18, if $\operatorname{proj}_{2}\left(K_{0}^{ \pm}\right)$is nontrivial then $K_{0}^{ \pm}=H_{0} \cdot S^{1}$ and hence has the form $\left\{\left(\beta\left(m_{ \pm} \theta\right), e^{i n_{ \pm} \theta}\right)\right\} \cdot H_{0}$ where $\beta(\theta)=\operatorname{diag}\left(e^{-i \theta}, e^{i \theta}, 1\right) \in \operatorname{SU}(3)$. In 
fact $K_{0}^{-}$must have this form, so assume $K_{0}^{-}=\left\{\left(\beta\left(m_{-} \theta\right), e^{i n_{-} \theta}\right)\right\} \cdot H_{0}$. The other possibility for $K_{0}^{+}$is $\mathrm{SU}(3) \times 1$, which does give $K_{0}^{+} / H_{0} \approx S^{5}$.

First suppose $K_{0}^{+}=\mathrm{SU}(3) \times 1$. Then from Corollary $1.9, K^{-}$is connected and $\pi_{1}(M) \approx \pi_{1}\left(G / K^{-}\right)$. It then follows that $n_{-}=1$, so $K^{-}=\left\{\left(\beta(m \theta), e^{i \theta}\right)\right\} \cdot H_{0}$ in this case. From Lemma $1.10, H=H_{0} \cdot \mathbb{Z}_{n}$ for $\mathbb{Z}_{n} \subset\left\{\left(\beta(m \theta), e^{i \theta}\right)\right\}$. The condition that $H \cap \mathrm{SU}(3) \times 1=H_{0}$ means that $\operatorname{gcd}(m, n)=1$. Therefore we get the family $Q_{G}^{7}$ of diagrams

$$
\begin{aligned}
& \mathrm{SU}(3) \times S^{1} \supset\left\{\left(\beta(m \theta), e^{i \theta}\right)\right\} \cdot H_{0}, \mathrm{SU}(3) \times \mathbb{Z}_{n} \supset H_{0} \cdot \mathbb{Z}_{n}, \\
& H_{0}=\mathrm{SU}(1) \mathrm{SU}(2) \times 1, \quad \mathbb{Z}_{n} \subset\left\{\left(\beta(m \theta), e^{i \theta}\right)\right\}, \\
& \beta(\theta)=\operatorname{diag}\left(e^{-i \theta}, e^{i \theta}, 1\right), \quad \operatorname{gcd}(m, n)=1 .
\end{aligned}
$$

Next assume $K_{0}^{ \pm}=\left\{\left(\beta\left(m_{ \pm} \theta\right), e^{i n_{ \pm} \theta}\right)\right\} \cdot H_{0}$. Notice that $\{\beta(\theta)\} \cap H_{0}=1$ and hence this situation satisfies the hypotheses of Lemma 4.2, for $L=\mathrm{U}(2)$. Then, by that lemma, we have precisely the family

$\left(N_{H}^{7}\right) \quad \mathrm{SU}(3) \times S^{1} \supset\left\{\left(\beta\left(m_{-} \theta\right), e^{i n_{-} \theta}\right)\right\} \cdot H,\left\{\left(\beta\left(m_{+} \theta\right), e^{i n_{+} \theta}\right)\right\} \cdot H \supset H$

$$
\begin{aligned}
H_{0} & =\mathrm{SU}(1) \operatorname{SU}(2) \times 1, \quad H=H_{-} \cdot H_{+}, K^{-} \neq K^{+}, \\
\beta(\theta) & =\operatorname{diag}\left(e^{-i \theta}, e^{i \theta}, 1\right), \quad \operatorname{gcd}\left(n_{-}, n_{+}, d\right)=1,
\end{aligned}
$$

where $d$ is the index of $H \cap K_{0}^{-} \cap K_{0}^{+}$in $K_{0}^{-} \cap K_{0}^{+}$,

and the family

$$
\begin{gathered}
\mathrm{SU}(3) \times S^{1} \supset\left\{\left(\beta(m \theta), e^{i \theta}\right)\right\} \cdot H_{0},\left\{\left(\beta(m \theta), e^{i \theta}\right)\right\} \cdot H_{0} \supset H_{0} \cdot \mathbb{Z}_{n} \\
H_{0}=\mathrm{SU}(1) \mathrm{SU}(2) \times 1, \quad \mathbb{Z}_{n} \subset\left\{\left(\beta(m \theta), e^{i \theta}\right)\right\}, \\
\beta(\theta)=\operatorname{diag}\left(e^{-i \theta}, e^{i \theta}, 1\right) .
\end{gathered}
$$

Now we address the remaining cases from Table $\mathrm{V}$.

Cases $3_{7}, 7_{7}, 10_{7}$ and $14_{7}$. In the first three cases, we have $G=G_{s s} \times T^{2}$, where $G_{s s}$ is semisimple and $\operatorname{rank}(H)=\operatorname{rank}\left(G_{s s}\right)$. Therefore by Proposition 1.18, the resulting actions must all be product actions.

In Case $14_{7}$ we see from Section 1.24 that $H_{1}=\mathrm{Sp}(2)$ is maximal among connected subgroups in SU(4). Therefore, Proposition 1.18 says $K^{-}, K^{+}$and $H$ are connected. Further, we can assume $K^{-}=H_{1} \times S^{1}$ and that $K^{+}$is either $H_{1} \times S^{1}$ or has the form $K_{1} \times 1$ for $K_{1} / H_{1} \approx S^{l}$. If $K^{+}=K_{1} \times 1$ then from Section 1.24, $K_{1}$ would have to be $\mathrm{SU}(4)$ and in this case we do have $\mathrm{SU}(4) / \mathrm{Sp}(2) \approx S^{5}$. Therefore we have the possibilities

$$
\mathrm{SU}(4) \times S^{1} \supset \mathrm{Sp}(2) \times S^{1}, \mathrm{Sp}(2) \times S^{1} \supset \mathrm{Sp}(2) \times 1,
$$

and

$$
\mathrm{SU}(4) \times S^{1} \supset \mathrm{Sp}(2) \times S^{1}, \mathrm{SU}(4) \times 1 \supset \mathrm{Sp}(2) \times 1,
$$


both of which give simply connected manifolds. The first is a product action and the second is a sum action.

Cases 97 and 117. In both cases $G=G_{1} \times S^{3}$ and $H_{0}=H_{1} \times S^{1}$, where $H_{1}$ is maximal among connected subgroups of $G_{1}$. Then $\operatorname{proj}_{1}\left(K_{0}^{ \pm}\right)$are either $H_{1}$ or $G_{1}$ and $\operatorname{proj}_{2}\left(K_{0}^{ \pm}\right)$are either $S^{1}$ or $S^{3}$. It is also clear that if $\operatorname{proj}_{2}\left(K_{0}^{ \pm}\right)=S^{3}$ then $K_{0}^{ \pm} \supset$ $1 \times S^{3}$ and so if $\operatorname{proj}_{1}\left(K_{0}^{ \pm}\right)=G_{1}$ then $K_{0}^{ \pm} \supset G_{1} \times 1$ as well. Therefore the proper subgroups $K_{0}^{ \pm}$must each be either $G_{1} \times S^{1}$ or $H_{1} \times S^{3}$. Note that $H_{1} \times S^{3} / H_{1} \times S^{1}$ is always a sphere. In Case $9_{7}, G_{1} \times S^{1} / H_{1} \times S^{1} \approx \mathrm{SU}(3) / \mathrm{U}(2) \approx \mathbb{C P}^{2}$ so this is not a possibility for $K^{ \pm}$but in Case $11_{7}, G_{1} \times S^{1} / H_{1} \times S^{1}$ is a sphere. Notice that in all cases $l_{ \pm}>1$ so $H, K^{-}$and $K^{+}$must all be connected by Corollary 1.9. Therefore we have these possible diagrams:

$$
\begin{gathered}
\mathrm{SU}(3) \times S^{3} \supset \mathrm{U}(2) \times S^{3}, \mathrm{U}(2) \times S^{3} \supset \mathrm{U}(2) \times S^{1}, \\
\mathrm{Sp}(2) \times S^{3} \supset \mathrm{Sp}(1) \mathrm{Sp}(1) \times S^{3}, \mathrm{Sp}(1) \mathrm{Sp}(1) \times S^{3} \supset \mathrm{Sp}(1) \mathrm{Sp}(1) \times S^{1}, \\
\mathrm{Sp}(2) \times S^{3} \supset \mathrm{Sp}(1) \mathrm{Sp}(1) \times S^{3}, \mathrm{Sp}(2) \times S^{1} \supset \mathrm{Sp}(1) \operatorname{Sp}(1) \times S^{1}, \\
\mathrm{Sp}(2) \times S^{3} \supset \mathrm{Sp}(2) \times S^{1}, \mathrm{Sp}(2) \times S^{1} \supset \mathrm{Sp}(1) \mathrm{Sp}(1) \times S^{1},
\end{gathered}
$$

all of which are simply connected by Lemma 1.10 . The third is a sum action and the remaining three actions are product actions.

Cases $12,1_{7}$ and $15_{7}$. In each of these cases, $H_{0}$ is maximal in $G$ among connected subgroups. Therefore, Proposition 1.22 gives a full description of these types of actions. Proposition 1.20 also deals with Case $15_{7}$ separately.

Case $1_{7}\left(G=S^{3} \times S^{3}\right)$. Here $H$ is discrete. From this it follows that for $K^{ \pm} / H$ to be spheres, $K_{0}^{ \pm}$must themselves be covers of spheres. From Section 1.24 we see that $K_{0}^{ \pm}$must be one of $\left\{\left(e^{x_{ \pm} p_{ \pm} \theta}, e^{y_{ \pm} q_{ \pm} \theta}\right)\right\}$ for $x_{ \pm}, y_{ \pm} \in \operatorname{Im}(\mathbb{H}), S^{3} \times 1,1 \times S^{3}$ or $\Delta_{g_{0}} S^{3}=\left\{\left(g, g_{0} g g_{0}^{-1}\right)\right\}$ for $g_{0} \in S^{3}$. We break this into cases of $K^{ \pm}$.

Case $1_{7} \mathbf{A}\left(K_{0}^{-} \approx S^{3}\right.$ and $\left.K_{0}^{+} \approx S^{3}\right)$. In this case we know from Corollary 1.9 that $H, K^{-}$and $K^{+}$must all be connected. Hence $N(H)_{0}=S^{3} \times S^{3}$ and we can conjugate $K^{-}$and $K^{+}$by anything in $S^{3} \times S^{3}$ without changing the manifold, by Proposition 1.2. In particular if $K^{ \pm}=\Delta_{g_{0}} S^{3}$ then we can assume $g_{0}=1$. Therefore we get the following possible groups diagrams up to automorphism of $G$, all of which are clearly simply connected by Lemma 1.10 :

$\left(Q_{A}^{7}\right)$

$\left(Q_{B}^{7}\right)$

$$
\begin{gathered}
S^{3} \times S^{3} \supset S^{3} \times 1, S^{3} \times 1 \supset 1, \\
S^{3} \times S^{3} \supset S^{3} \times 1,1 \times S^{3} \supset 1, \\
S^{3} \times S^{3} \supset S^{3} \times 1, \Delta S^{3} \supset 1, \\
S^{3} \times S^{3} \supset \Delta S^{3}, \Delta S^{3} \supset 1
\end{gathered}
$$

The first of these actions is a product action and the second is a sum action. 
Case $1_{7} \mathbf{B}\left(K_{0}^{-} \approx S^{1}\right.$ and $\left.K_{0}^{+} \approx S^{3}\right)$. From Lemma 1.10 , we know that $K^{-}$is connected and $H=\mathbb{Z}_{n} \subset K^{-}$such that $H \cap K_{0}^{+}=1$. After conjugation of $G$ we can assume that $K^{-}=\left\{\left(e^{i p \theta}, e^{i q \theta}\right)\right\}$. If $K_{0}^{+}=S^{3} \times 1$ then the condition $H \cap K_{0}^{+}=1$ means that $n$ and $q$ are relatively prime. Therefore we have this family of diagrams:

$\left(N_{C}^{7}\right) \quad S^{3} \times S^{3} \supset\left\{\left(e^{i p \theta}, e^{i q \theta}\right)\right\}, S^{3} \times \mathbb{Z}_{n} \supset Z_{n}, \quad$ where $(q, n)=1$

which all give simply connected manifolds by Lemma 1.10 .

Next suppose that $K_{0}^{+}=\Delta_{g_{0}} S^{3}$ for some $g_{0} \in S^{3}$. Notice that $N\left(K_{0}^{+}\right)=$ $\left\{\left( \pm g, g_{0} g g_{0}^{-1}\right)\right\}$ and since $L \subset N\left(L_{0}\right)$ for every subgroup $L$, it follows that $K^{+}$ can have at most two components and hence $H$ can have at most two elements. In particular this means that $H$ is normal in $G$ and hence by Proposition 1.2, we can conjugate $K^{+}$by $\left(1, g_{0}^{-1}\right)$ without changing the resulting manifold. Lastly, if $n=2$ the condition that $H \cap K_{0}^{+}=1$ means that $p$ and $q$ are not both odd and not both even since $(p, q)=1$. Without loss of generality we can assume that $p$ is even and $p$ is odd. Therefore we have the following family of diagrams, all of which are simply connected by Lemma 1.10 :

$\left(P_{D}^{7}\right) \quad S^{3} \times S^{3} \supset\left\{\left(e^{i p \theta}, e^{i q \theta}\right)\right\}, \Delta S^{3} \cdot \mathbb{Z}_{n} \supset Z_{n}$

where $(p, q)=1$; and $n$ is 1 and $p$ and $q$ arbitrary, or $n$ is 2 and $p$ even.

Case $1_{7} \mathbf{C}\left(K_{0}^{-} \approx S^{1}\right.$ and $\left.K_{0}^{+} \approx S^{1}\right)$. Here we have $K_{0}^{ \pm}=\left\{\left(e^{x_{ \pm} p_{ \pm} \theta}, e^{y_{ \pm} q_{ \pm} \theta}\right)\right\}$. To address this case we will break it up into further cases depending on how big the group generated by $K_{0}^{-}$and $K_{0}^{-}$is.

Case $1_{7} \mathbf{C 1}$ ( $K_{0}^{-}$and $K_{0}^{+}$are both contained in some torus). After conjugation we can assume that $K_{0}^{ \pm}=\left\{\left(e^{i p_{ \pm} \theta}, e^{i q_{ \pm} \theta}\right)\right\}$. By Lemma $1.10, H=H_{-} \cdot H_{+}$, where $H_{ \pm}=\mathbb{Z}_{n_{ \pm}} \subset K_{0}^{ \pm}$, and conversely by Lemma 1.10 , such groups will always give simply connected manifolds. Therefore we have these possibilities: .

$\left(N_{A}^{7}\right) \quad S^{3} \times S^{3} \supset\left\{\left(e^{i p_{-} \theta}, e^{i q_{-} \theta}\right)\right\} \cdot H_{+},\left\{\left(e^{i p_{+} \theta}, e^{i q_{+} \theta}\right)\right\} \cdot H_{-} \supset H_{-} \cdot H_{+}$,

where $H_{ \pm}=\mathbb{Z}_{n_{ \pm}} \subset K_{0}^{ \pm}$.

Case ${ }_{7} \mathbf{C 2}\left(K_{0}^{-}\right.$and $K_{0}^{+}$are both contained in $\left.S^{3} \times 1\right)$. Here it follows from Lemma 1.10 that $H, K^{-}$and $K^{+}$are all contained in $S^{3} \times 1$. It also follows from Lemma 1.10 that $M^{7}$, given by the diagram $G \supset K^{-}, K^{+} \supset H$, will be simply connected if and only if the manifold $N^{4}$ given by the diagram $S^{3} \times 1 \supset K^{-}, K^{+} \supset H$ is simply connected. Therefore this gives a product action.

Case $1_{7} \mathrm{C3}\left(K_{0}^{-}\right.$and $K_{0}^{+}$are both contained in $S^{3} \times S^{1}$ but not in $T^{2}$ or $\left.S^{3} \times 1\right)$. It follows from Lemma 1.10 that $H, K^{-}$and $K^{+}$must all be contained in $S^{3} \times S^{1}$ in this case. Notice further that if both $p_{-} q_{-}=0$ and $p_{+} q_{+}=0$, then we would be back in one of the previous cases. So after conjugation of $G$ and switching of and + , we can assume that $K_{0}^{-}=\left\{\left(e^{i p_{-} \theta}, e^{i q_{-} \theta}\right)\right\}$, where $p_{-} q_{-} \neq 0$. For $K_{0}^{+}$we 
can assume that $y_{+}=i$ and denote $x_{+}=x$. It also follows that $p_{+} \neq 0$ and $x \neq \pm i$ since otherwise we would be in a previous case again.

Notice that $N\left(K_{0}^{-}\right)=\left\{\left(e^{i \theta}, e^{i \phi}\right)\right\} \cup\left\{\left(j e^{i \theta}, j e^{i \phi}\right)\right\}$ and $K^{-} \subset S^{3} \times S^{1}$ and hence $K^{-} \subset\left\{\left(e^{i \theta}, e^{i \phi}\right)\right\}$. Similarly if $q_{+} \neq 0$ then $N\left(K_{0}^{+}\right)=\left\{\left(e^{x \theta}, e^{i \phi}\right)\right\} \cup\left\{\left(w e^{x \theta}, j e^{i \phi}\right)\right\}$ for $w \in x^{\perp} \cap \operatorname{Im} S^{3}$. Therefore $K^{+} \subset\left\{\left(e^{x \theta}, e^{i \phi}\right)\right\}$ in this case as well. However $H$ would then be a subset of the intersection of these two sets, $H \subset\left\{\left( \pm 1, e^{i \phi}\right)\right\}$, and $N(H)_{0}$ would contain $S^{3} \times 1$. We would then be able to conjugate $K^{+}$into the set $\left\{\left(e^{i \theta}, e^{i \phi}\right)\right\}$ without changing the resulting manifold, by Proposition 1.2. This would put us back into Case $1_{7} \mathrm{C} 1$, so we can assume that $q_{+}=0$ and $K_{0}^{+}=\left\{\left(e^{x \theta}, 1\right)\right\}$.

Therefore $N\left(K_{0}^{+}\right)=\left(\left\{e^{x \theta}\right\} \cup\left\{w e^{x \theta}\right\}\right) \times S^{3}$. Again we see for $N\left(K_{0}^{-}\right) \cap N\left(K_{0}^{+}\right) \nsubseteq$ $\left\{\left( \pm 1, e^{i \phi}\right)\right\}$ we need $x \perp i$. So after conjugation we can assume $K_{0}^{+}=\left\{\left(e^{j \theta}, 1\right)\right\}$. Then $H \subset\{ \pm 1, \pm i\} \times S^{1}$. By Lemma $1.10, H=H_{-} \cdot H_{+}$for $H_{ \pm}=\mathbb{Z}_{n_{ \pm}} \subset K_{0}^{ \pm}$. We see then that $n_{+}$is 1 or 2 and the conditions that $H \subset\{ \pm 1, \pm i\} \times S^{1}$ but $H \nsubseteq\{ \pm 1\} \times S^{1}$ mean that $4 \mid n_{-}$and $p_{-} \equiv \pm n_{-} / 4 \bmod n_{-}$. Conversely we see we get the following possible diagrams:

$$
\left(N_{B}^{7}\right) \quad S^{3} \times S^{3} \supset\left\{\left(e^{i p \theta}, e^{i q \theta}\right)\right\} \cdot H_{+},\left\{\left(e^{j \theta}, 1\right)\right\} \cdot H_{-} \supset H_{-} \cdot H_{+},
$$

where $H_{ \pm}=\mathbb{Z}_{n_{ \pm}} \subset K_{0}^{ \pm}, n_{+} \leq 2,4 \mid n_{-}$and $p \equiv \pm \frac{1}{4} n_{-} \bmod n_{-}$,

all of which give simply connected manifolds by Lemma 1.10.

Case $1_{7} \mathbf{C 4}\left(K_{0}^{-}\right.$and $K_{0}^{+}$are not both contained in $S^{3} \times S^{1}$ or $\left.S^{1} \times S^{3}\right)$. As in the previous case, we can assume here that both $p_{-} q_{-} \neq 0$ and $p_{+} q_{+} \neq 0$ and after conjugation $K_{0}^{-}=\left\{\left(e^{i p_{-} \theta}, e^{i q_{-} \theta}\right)\right\}$ and $K_{0}^{+}=\left\{\left(e^{x p_{+} \theta}, e^{y q_{+} \theta}\right)\right\}$. Then if $u \in$ $x^{\perp} \cap \operatorname{Im} S^{3}$ and $w \in y^{\perp} \cap \operatorname{Im} S^{3}$ then we have $N\left(K_{0}^{-}\right)=\left\{\left(e^{i \theta}, e^{i \phi}\right)\right\} \cup\left\{\left(j e^{i \theta}, j e^{i \phi}\right)\right\}$, $N\left(K_{0}^{+}\right)=\left\{\left(e^{x \theta}, e^{y \phi}\right)\right\} \cup\left\{\left(u e^{x \theta}, v e^{y \phi}\right)\right\}$ and $H \subset N\left(K_{0}^{-}\right) \cap N\left(K_{0}^{+}\right)$.

We now claim that we can assume $x$ and $i$ are perpendicular. Suppose they are not. Then if we denote the two elements in $i^{\perp} \cap x^{\perp} \cap \operatorname{Im} S^{3}$ by $\pm w$, we would have $H \subset\{ \pm 1, \pm w\} \times S^{3}$. Notice that conjugation by $\left(e^{w \alpha}, 1\right)$ fixes $\{ \pm 1, \pm w\} \times S^{3}$ pointwise and hence $\left(e^{w \alpha}, 1\right) \in N(H)_{0}$ for all $\alpha \in \mathbb{R}$. Therefore, by Proposition 1.2 we can conjugate $K^{+}$by $\left(e^{w \alpha}, 1\right)$ without changing the resulting manifold. Since $w \perp\{x, i\}$, conjugation by $\left(e^{w \alpha}, 1\right)$ fixes the $1 w$-space and rotates the $i x$-space by $2 \alpha$. So for the right choice of $\alpha$ we can rotate $x$ into $i$. Therefore we could assume that $K_{0}^{+}=\left\{\left(e^{i p_{+} \theta}, e^{y q_{+} \theta}\right)\right\}$, bringing us back to an earlier case. Hence we can assume that $x \perp i$ and similarly $y \perp i$. Then after conjugation of $G$ we can take $K_{0}^{+}=\left\{\left(e^{j p_{+} \theta}, e^{j q_{+} \theta}\right)\right\}$, without affecting $K^{-}$.

Then the condition $H \subset N\left(K_{0}^{+}\right) \cap N\left(K_{0}^{-}\right)$becomes 
where

$Q=\{ \pm 1, \pm i, \pm j, \pm k\}$ and $\Delta_{-} Q=\{ \pm(1,-1), \pm(i,-i), \pm(j,-j), \pm(k,-k)\}$.

In particular, if $\left(h_{1}, h_{2}\right) \in H$ then $h_{1}= \pm h_{2}$.

We also know from Lemma 1.10 that $H$ is generated by $H \cap K_{0}^{-}=: H_{-}$and $H \cap K_{0}^{+}=: H_{+}$, where $H_{ \pm}$are both cyclic subgroups of the circles $K_{0}^{ \pm}$. Let $h_{ \pm}=\left(h_{1}^{ \pm}, h_{2}^{ \pm}\right)$be generators of $H_{ \pm}$, so that $h_{-}$and $h_{+}$generate $H$. If both $h_{ \pm}$ were to have order 1 or 2 , then $H$ would be contained in $\{ \pm 1\} \times\{ \pm 1\}$ and we would be back in a previous case, as before. So assume that $h_{-}$has order 4 . After conjugation of $G$ we can assume that $h_{-}=(i, i)$. The condition that $h_{-} \in K_{0}^{-}$ means that $p_{-}, q_{-} \equiv \pm 1 \bmod 4$; however, after switching the sign of both $p_{-}$ and $q_{-}$, we can assume that $p_{-}, q_{-} \equiv 1 \bmod 4$.

We will now break our study into further cases depending on the order of $h_{+}$, which is either 1,2 or 4 .

Case $1_{7} \mathbf{C 4 a}\left(h_{+} \in\langle(i, i)\rangle\right)$. Then $H=\langle(i, i)\rangle$. Hence we get the family

$\left(P_{A}^{7}\right) \quad S^{3} \times S^{3} \supset\left\{\left(e^{i p_{-} \theta}, e^{i q_{-} \theta}\right)\right\},\left\{\left(e^{j p_{+} \theta}, e^{j q_{+} \theta}\right)\right\} \cdot H \supset\langle(i, i)$,

where $p_{-}, q_{-} \equiv 1 \bmod 4$.

Case $1_{7} \mathbf{C 4 b}\left(\#\left(h_{+}\right)=2\right.$ but $\left.h_{+} \notin\langle(i, i)\rangle\right)$. It follows that $h_{+}$must be $(1,-1)$ or $(-1,1)$; after switching the factors of $G=S^{3} \times S^{3}$ we can assume the former. The condition that $h_{+} \in K_{0}^{+}$means that $p_{+}$is even. Therefore we have the following family of possibilities:

$\left(P_{B}^{7}\right) \quad S^{3} \times S^{3} \supset\left\{\left(e^{i p_{-} \theta}, e^{i q_{-} \theta}\right)\right\} \cdot H,\left\{\left(e^{j p_{+} \theta}, e^{j q_{+} \theta}\right)\right\} \cdot H \supset\langle(i, i),(1,-1)\rangle$, where $p_{-}, q_{-} \equiv 1 \bmod 4$ and $p_{+}$is even.

Case $1_{7} \mathbf{C 4 c}\left(\#\left(h_{+}\right)=4\right)$. In this last case, $h_{+}$must be one of $(j, j),(j,-j)$, $(-j, j)$ or $(-j,-j)$; after conjugation of $G$ by $( \pm i, \pm i)$ we can assume the first. As before, the condition that $h_{+} \in K_{0}^{+}$means that $p_{+}, q_{+} \equiv \pm 1 \bmod 4$ but we can assume that $p_{+}, q_{+} \equiv 1 \bmod 4$, after a change of signs on $p_{+}$and $q_{+}$. Then $H=\Delta Q$ and we have the possibilities

$\left(P_{C}^{7}\right) \quad S^{3} \times S^{3} \supset\left\{\left(e^{i p_{-} \theta}, e^{i q_{-} \theta}\right)\right\} \cdot H,\left\{\left(e^{j p_{+} \theta}, e^{j q_{+} \theta}\right)\right\} \cdot H \supset \Delta Q$,

where $p_{ \pm}, q_{ \pm} \equiv 1 \bmod 4$.

By Lemma 1.10, all of the diagrams above do give simply connected manifolds.

Case $4_{7}(G=\mathrm{SU}(3))$. In this case, $H_{0}=T^{2}$. From Section 1.24, the proper subgroups $K_{0}^{ \pm}$must both be U(2) up to conjugacy. It then follows from Corollary 1.9 that $H, K^{-}$and $K^{+}$are all connected. Now fix $H=\operatorname{diag}(\operatorname{SU}(3)) \approx T^{2}$. If $K^{ \pm}$ contains this $T^{2}$, then it must be a conjugate of $\mathrm{U}(2)$ by an element of the Weyl group $W=N\left(T^{2}\right) / T^{2}$. We see that there are precisely three such conjugates of 
$\mathrm{U}(2)$ and they are permuted by the elements of $W$. Therefore, there are two possibilities for the pair $K^{-}, K^{+}$up to conjugacy of $G$ : $\mathrm{S}(\mathrm{U}(1) \mathrm{U}(2)), \mathrm{S}(\mathrm{U}(1) \mathrm{U}(2))$ or $\mathrm{S}(\mathrm{U}(1) \mathrm{U}(2)), \mathrm{S}(\mathrm{U}(2) \mathrm{U}(1))$. This gives us precisely these two simply connected diagrams:

$\left(N_{G}^{7}\right)$

$$
\mathrm{SU}(3) \supset \mathrm{S}(\mathrm{U}(1) \mathrm{U}(2)), \mathrm{S}(\mathrm{U}(1) \mathrm{U}(2)) \supset T^{2} \text {, }
$$

and

$$
\mathrm{SU}(3) \supset \mathrm{S}(\mathrm{U}(1) \mathrm{U}(2)), \mathrm{S}(\mathrm{U}(2) \mathrm{U}(1)) \supset T^{2} \text {. }
$$

Case $5_{7}\left(G=S^{3} \times S^{3} \times S^{3}\right)$. It is clear that if $\operatorname{proj}_{1}\left(K_{0}^{ \pm}\right) \neq S^{1}$, then $K_{0}^{ \pm} \supset S^{3} \times 1 \times 1$ and similarly for the other factors. Hence each $K_{0}^{ \pm}$will be a product of $S^{3}$ factors and $S^{1}$ factors. Further, it is clear that for $K_{0}^{ \pm} / H$ to be a sphere we need $K_{0}^{ \pm}$to be one of $S^{3} \times S^{1} \times S^{1}, S^{1} \times S^{3} \times S^{1}$ or $S^{1} \times S^{1} \times S^{3}$. Then by Corollary 1.9, all of $H, K^{-}$and $\mathrm{K}^{+}$must be connected. Putting this together, we see we have the following possible simply connected diagrams, up to $G$-automorphism:

$$
\begin{aligned}
& S^{3} \times S^{3} \times S^{3} \supset S^{3} \times S^{1} \times S^{1}, S^{3} \times S^{1} \times S^{1} \supset S^{1} \times S^{1} \times S^{1}, \\
& S^{3} \times S^{3} \times S^{3} \supset S^{3} \times S^{1} \times S^{1}, S^{1} \times S^{3} \times S^{1} \supset S^{1} \times S^{1} \times S^{1} .
\end{aligned}
$$

It is clear that both of these are product actions.

Case $8_{7}(G=\mathrm{Sp}(2))$. Here $H_{0}$ is either $\mathrm{U}(2)_{\max }=\{\operatorname{diag}(z g, \bar{z} g)\}$ or $\mathrm{Sp}(1) \mathrm{SO}(2)$. Since $\mathrm{U}(2)_{\max }$ is maximal among connected subgroups, and $\mathrm{Sp}(2) / \mathrm{U}(2)_{\max }$ is not a sphere, we see this is not a possibility for $H_{0}$. So assume $H_{0}=\operatorname{Sp}(1) \operatorname{SO}(2)$. Then from Section 1.24, we see the proper subgroups $K_{0}^{ \pm}$must be conjugates of $\mathrm{Sp}(1) \mathrm{Sp}(1)$. Since the only conjugate of $\mathrm{Sp}(1) \mathrm{Sp}(1)$ that contains $\mathrm{Sp}(1) \mathrm{SO}(2)$ is the usual $\mathrm{Sp}(1) \operatorname{Sp}(1)$, we see $K_{0}^{ \pm}=\operatorname{Sp}(1) \operatorname{Sp}(1)$. Then by Corollary $1.9, H, K^{-}$ and $K^{+}$must all be connected. Therefore we get the one possible diagram:

$\left(N_{I}^{7}\right)$

$$
\mathrm{Sp}(2) \supset \mathrm{Sp}(1) \mathrm{Sp}(1), \operatorname{Sp}(1) \mathrm{Sp}(1) \supset \mathrm{Sp}(1) \mathrm{SO}(2) .
$$

\section{Identifying some actions}

Here we will identify many of the actions arising from the classification. We will review what is known about the remaining unidentified actions.

5.1. Isometric actions on symmetric spaces. In this section we will list all isometric cohomogeneity one actions on compact simply connected symmetric spaces of dimension seven or less. Hsiang and Lawson [1971] classified cohomogeneity one actions on symmetric spheres in (see [Straume 1996] for correction) and later Uchida [1977] did the same for complex projective spaces. Kollross [2002] generalized these results to a classification of cohomogeneity one actions on irreducible symmetric spaces of compact type. 
The only maximal isometric cohomogeneity one actions on compact simply connected irreducible symmetric spaces of dimension 7 or less are the following, up to equivalence: the sum actions of $\mathrm{SO}\left(k_{1}\right) \times \mathrm{SO}\left(k_{2}\right)$ on $S^{k_{1}+k_{2}-1} \subset \mathbb{R}^{k_{1}} \times \mathbb{R}^{k_{2}}$ for $k_{i} \geq 1$; the tensor actions of $\mathrm{SO}(k) \times \mathrm{SO}(2)$ on $S^{2 k-1} \subset \mathbb{R}^{k \times 2}$ via $(A, B) \star M=$ $A M B^{-1}$ for $k=3,4$; the irreducible linear action of $\mathrm{SO}(3)$ on $S^{4}$, the $\mathrm{SU}(3)$ action on $S^{7} \subset \mathfrak{s u}(3)$ via Ad, and the $\mathrm{SO}(4)$ action on $S^{7}$ via the isotropy representation of $\mathrm{G}_{2} / \mathrm{SO}(4)$; the linear actions on complex projective spaces of $\mathrm{U}(n)$ or $\mathrm{SO}(n+1)$ on $\mathbb{C P}^{n}=\mathrm{SU}(n+1) / \mathrm{U}(n)$, or $\mathrm{S}(\mathrm{U}(2) \times \mathrm{U}(2))$ on $\mathbb{C P}^{3}=\mathrm{SU}(4) / \mathrm{U}(3)$; and the two remaining symmetric space actions of $\mathrm{U}(2)$ on $\mathrm{SU}(3) / \mathrm{SO}(3)$ and $\mathrm{SO}(4)$ on $\mathrm{SO}(5) / \mathrm{SO}(2) \mathrm{SO}(3)$. Here, a maximal cohomogeneity one action is one that cannot be extended to a cohomogeneity one action by a larger connected group.

Several actions do not appear in this list since they are equivalent to actions that do appear. For example, the complex tensor action of $\mathrm{SU}(2) \times \mathrm{U}(2)$ on $S^{7} \subset \mathbb{C}^{2 \times 2}$ via $(A, B) \star M=A M B^{-1}$ is equivalent to the real tensor action of $\mathrm{SO}(4) \times \mathrm{SO}(2)$ on $S^{7} \subset \mathbb{R}^{4 \times 2}$.

For each action of $G$ on $M$, it is not difficult to see which subgroups of $G$ act on $M$ with the same orbits. Many of these actions are simply sum actions or fixedpoint actions as described in Section 1.21. Since we have already examined these special cases we will not address them again here. In Table VI we list the remaining nonreducible cohomogeneity one actions on irreducible symmetric spaces.

For a complete list of cohomogeneity one actions on compact simply connected symmetric spaces we must only find such actions on product symmetric spaces. By looking at each such product individually, considering its full isometry group, then determining which subgroups of the isometry group can act by cohomogeneity one on the product, we can get a list of all possible actions. Many of these actions will be simple product actions, and since we have already addressed those in general in Section 1.21, we will not consider them again here. The remaining actions that are also nonreducible are listed in Tables VII and VIII.

In Tables VI, VII and VIII, we also list the families these actions belong to. These families are easily determined by computing the group diagrams for each action. The group diagram for the last entry in Table VI is described [Grove et al. 2008, Section 4]. In one case, the action is equivalent to a product action, even though the action itself is not a product action; here the word "product" is printed in the left-hand column.

5.2. Brieskorn varieties. The group $S^{1} \times \mathrm{SO}(n)$ has a well-known cohomogeneity one action on the Brieskorn variety

$$
B_{d}^{2 n-1}=\left\{\left.z \in \mathbb{C}^{n+1}\left|z_{0}^{d}+z_{1}^{2}+z_{2}^{2}+\cdots+z_{n}^{2}=0, \sum_{i=0}^{n}\right| z_{i}\right|^{2}=1\right\},
$$

given by $(w, A) \star\left(z_{0}, z_{1}, z_{2}, \ldots, z_{n}\right)=\left(w^{2} z_{0}, w^{d} A\left(z_{1}, z_{2}, \ldots, z_{n}\right)^{t}\right)$. 
$Q_{B}^{5}$ (part) $\mathrm{SO}(3) \times \mathrm{SO}(2)$ on $S^{5} \subset \mathbb{R}^{3 \times 2}$ via $(A, B) \star M=A M B^{-1}$

$P^{5}$ (part) $\mathrm{U}(2)$ on $\mathrm{SU}(3) / \mathrm{SO}(3)$

$Q_{C}^{5}$ (all) $S^{3} \times S^{1}$ on $S^{5} \subset \mathbb{H} \times \mathbb{C}$ via $(g, z) \star(p, w)=\left(g p \bar{z}^{n}, z^{m} w\right)$

$Q_{A}^{6}$ (all) $\quad \begin{aligned} & \mathrm{SO}(4) \text { on } \mathbb{C P}^{3}=\mathrm{SU}(4) / \mathrm{U}(3) \\ & \mathrm{SO}(4) \text { on } \mathrm{SO}(5) / \mathrm{SO}(2) \mathrm{SO}(3)\end{aligned}$

$\mathrm{SO}(4)$ on $\mathrm{SO}(5) / \mathrm{SO}(2) \mathrm{SO}(3)$

$Q_{C}^{6}($ all $) \quad S^{3} \times S^{3}$ on $S^{6} \subset \mathbb{H} \times \operatorname{Im}(\mathbb{M})$ via $\left(g_{1}, g_{2}\right) \star(p, q)=\left(g_{1} p g_{2}^{-1}, g_{2} q g_{2}^{-1}\right)$

$Q_{D}^{6}($ all $) \quad \mathrm{SU}(2) \times \mathrm{SU}(2)$ on $\mathbb{C P}^{3}=\mathrm{SU}(4) / \mathrm{U}(3)$

$Q_{A}^{7}$ (all) $S^{3} \times S^{3}$ on $S^{7} \subset \mathbb{H} \times \mathbb{W}$ via $\left(g_{1}, g_{2}\right) \star(p, q)=\left(g_{1} p g_{2}^{-1}, g_{2} q\right)$

$Q_{D}^{7}$ (part) $\mathrm{SO}(4) \times \mathrm{SO}(2)$ on $S^{7} \subset \mathbb{R}^{4 \times 2}$ via $(A, B) \star M=A M B^{-1}$

$Q_{E}^{7}$ (all) $\mathrm{SU}(3)$ on $S^{7} \subset \mathfrak{s u}(3)$ via Ad

$Q_{G}^{7}$ (all) $\mathrm{SU}(3) \times S^{1}$ on $S^{7} \subset \mathbb{C}^{3} \times \mathbb{C}$ via $(A, z) \star(x, w)=\left(z^{n} A x, z^{m} w\right)$

$P_{C}^{7}$ (part) $\mathrm{SO}(4)$ on $S^{7}$ via the isotropy representation of $\mathrm{G}_{2} / \mathrm{SO}(4)$

Table VI. Nonreducible isometric cohomogeneity one actions on compact simply connected irreducible symmetric spaces in dimensions 5, 6 and 7 that are not sum actions or fixed-point actions. Also indicated is whether the family of actions listed in the right column makes up all or part of the family listed in the left.

These actions were extensively studied in [Grove et al. 2006]. In particular they describe the group diagrams for the actions. In dimension 5 the group diagrams are

$$
\begin{aligned}
& S^{3} \times S^{1} \supset\left\{\left(e^{i \theta}, 1\right)\right\} \cdot H,\left\{\left(e^{j d \theta}, e^{2 i \theta}\right)\right\} \supset\langle(j,-1)\rangle \text { for } d \text { odd, } \\
& S^{3} \times S^{1} \supset\left\{\left(e^{i \theta}, 1\right)\right\},\left\{\left(e^{j d \theta}, e^{i \theta}\right)\right\} \supset 1 \quad \text { for } d \text { even, }
\end{aligned}
$$

where we have taken a more effective version of the diagram in the second case. The first diagram is precisely the diagram of $Q_{B}^{5}$ for $d=p$ and hence $Q_{B}^{5} \approx B_{d}^{5}$ for $d$ odd. Since $H$ is trivial in the second diagram, Proposition 1.2 says this diagram is equivalent to one of type $N^{5}$ for certain parameters.

In dimension 7, after lifting the action to $S^{3} \times S^{3} \times S^{1}$, the group diagrams are given by

$$
S^{3} \times S^{3} \times S^{1} \supset\left\{\left(e^{i \phi}, e^{i \phi} e^{i d \theta}, e^{i \theta}\right)\right\}, \pm \Delta S^{3} \times \pm 1 \supset \pm \Delta S^{1} \times \pm 1 \quad \text { if } d \text { is odd },
$$

where the $\pm \Delta S^{3}=\{(g, \pm g)\}$ and where the \pm signs are correlated, and

$$
S^{3} \times S^{3} \times S^{1} \supset\left\{\left(e^{i \phi}, e^{i \phi} e^{i d \theta}, e^{2 i \theta}\right)\right\}, \Delta S^{3} \times 1 \supset \Delta S^{1} \times 1 \quad \text { if } d \text { is even. }
$$




\begin{tabular}{|c|c|}
\hline $\begin{array}{c}Q_{A}^{5} \\
(\text { part })\end{array}$ & $\begin{aligned} S^{3} \times S^{1} \text { on } S^{2} \times S^{3} \subset \operatorname{Im}(\mathbb{M}) \times \mathbb{W} & \\
& \operatorname{via}(g, z) \star(p, q)=\left(g p g^{-1}, g q z^{-1}\right)\end{aligned}$ \\
\hline $\begin{array}{c}Q_{A}^{5} \\
(\text { all })\end{array}$ & $\begin{aligned} S^{3} \times S^{1} \text { on } S^{2} \times S^{3} \subset & \operatorname{Im}(\mathbb{H}) \times \mathbb{U} \\
& \operatorname{via}(g, z) \star(p, q)=\left(z^{n} p \bar{z}^{n}, g q \bar{z}^{m}\right)\end{aligned}$ \\
\hline $\begin{array}{c}N_{A}^{6} \\
\text { (all [Hoelscher 2010a]) }\end{array}$ & $\begin{array}{l}S^{3} \times T^{2} \text { on } S^{3} \times S^{3} \subset \mathbb{M} \times \mathbb{M} \\
\quad \operatorname{via}(g, z, w) \star(p, q)=\left(z^{a} w^{b} p \bar{z}^{c} \bar{w}^{d}, g q \bar{z}^{n} \bar{w}^{m}\right)\end{array}$ \\
\hline $\begin{array}{c}N_{A}^{6} \\
\text { (part) }\end{array}$ & $\begin{aligned} S^{3} \times T^{2} \text { on } S^{3} \times S^{3} \subset \mathbb{M} \times \mathbb{M} & \\
& \text { via }(g, z, w) \star(p, q)=(g p \bar{z}, g q \bar{w})\end{aligned}$ \\
\hline $\begin{array}{c}Q_{B}^{6} \\
(\text { all })\end{array}$ & $\begin{array}{l}S^{3} \times S^{3} \text { on } S^{3} \times S^{3} \subset \mathbb{M} \times \mathbb{M} \\
\quad \text { via }\left(g_{1}, g_{2}\right) \star(p, q)=\left(g_{1} p g_{1}^{-1}, g_{1} q g_{2}^{-1}\right)\end{array}$ \\
\hline$Q_{B}^{6}($ all $)$ & $\mathrm{SO}(4)$ on $S^{3} \times S^{3} \quad$ via $A \star(x, y)=(A x, A y)$ \\
\hline $\begin{array}{l}N_{E}^{6} \\
\text { (part) }\end{array}$ & $\begin{array}{l}S^{3} \times S^{3} \text { on } S^{2} \times S^{4} \subset \operatorname{Im}(\mathbb{M}) \times(\mathbb{H} \times \mathbb{R}) \\
\quad \operatorname{via}\left(g_{1}, g_{2}\right) \star(p, q, t)=\left(g_{1} p g_{1}^{-1}, g_{1} q g_{2}^{-1}, t\right)\end{array}$ \\
\hline product & $\begin{aligned} S^{3} \times S^{3} \text { on } S^{3} \times S^{4} \subset \mathbb{W} \times(\mathbb{N} \times \mathbb{R}) \\
\\
\operatorname{via}\left(g_{1}, g_{2}\right) \star(p, q, t)=\left(g_{1} p, g_{1} q g_{2}^{-1}, t\right)\end{aligned}$ \\
\hline $\begin{array}{c}N_{A}^{7} \\
\text { (part) }\end{array}$ & $\begin{array}{l}S^{3} \times S^{3} \text { on } S^{2} \times S^{2} \times S^{3} \subset \operatorname{Im}(\mathbb{M}) \times \operatorname{Im}(\mathbb{M}) \times \mathbb{M} \\
\text { via }\left(g_{1}, g_{2}\right) \star\left(p_{1}, p_{2}, q\right)=\left(g_{1} p_{1} g_{1}^{-1}, g_{1} p_{2} g_{1}^{-1}, g_{1} q g_{2}^{-1}\right)\end{array}$ \\
\hline $\begin{array}{c}N_{A}^{7} \\
\text { (part) }\end{array}$ & $\begin{array}{l}S^{3} \times S^{3} \text { on } S^{2} \times S^{2} \times S^{3} \subset \operatorname{Im}(\mathbb{M}) \times \operatorname{Im}(\mathbb{M}) \times \mathbb{M} \\
\text { via }\left(g_{1}, g_{2}\right) \star\left(p_{1}, p_{2}, q\right)=\left(g_{1} p_{1} g_{1}^{-1}, g_{2} p_{2} g_{2}^{-1}, g_{1} q g_{2}^{-1}\right)\end{array}$ \\
\hline
\end{tabular}

Table VII. Nonreducible isometric cohomogeneity one actions on compact simply connected products of irreducible symmetric spaces in dimensions 5, 6 and 7; these are not product actions. Also indicated is whether the family of actions listed in the righthand column makes up all or part of the family listed in the left. (First of two tables.)

This first diagram is exactly diagram $Q_{D}^{7}$ in the case that $n=2$ for $d=b$, since if $n=2$, then $b$ must be odd for the diagram to be effective. The second diagram above, is exactly $Q_{D}^{7}$ in the case $n=1$ since if $d$ is even we can take $d=2 b$ for $b$ in diagram $Q_{D}^{7}$. So the family $Q_{D}^{7}$ exactly corresponds to these actions on the Brieskorn varieties.

5.3. Important actions in more detail. We will now look at each of the actions in Tables I and II and summarize various facts that we have collected about them.

Primitive actions of Table I. 


\begin{tabular}{|c|c|c|}
\hline $\begin{array}{l}P_{A}^{7} \\
\text { part) }\end{array}$ & $S^{3} \times S^{3}$ on $S^{3} \times \mathbb{C} \mathbb{P}^{2}$ & $\begin{array}{l}\text { via }\left(g_{1}, g_{2}\right) \star(p, x)=\left(g_{1} p g_{2}^{-1}, g_{2} \star_{1} x\right) \\
\text { where } \star_{1} \text { is the action of } \operatorname{SO}(3) \text { on } \mathbb{C} \mathbb{P}^{2}\end{array}$ \\
\hline $\begin{array}{l}P_{C}^{7} \\
\text { art) }\end{array}$ & $\begin{array}{r}S^{3} \times S^{3} \text { on } S^{3} \times S^{4} \subset \\
\text { where }\end{array}$ & $\begin{array}{l}\text { via }\left(g_{1}, g_{2}\right) \star(p, y)=\left(g_{1} p g_{2}^{-1}, g_{2} \star_{1} y\right) \\
\text { irreducible linear action of } \mathrm{SO}(3) \text { on } S^{4}\end{array}$ \\
\hline $\begin{array}{l}P_{D}^{7} \\
\text { part) }\end{array}$ & $S^{3} \times S^{3}$ on $S^{3} \times \mathbb{C} \mathbb{P}^{2}$ & $\begin{array}{l}\text { via }\left(g_{1}, g_{2}\right) \star(p, x)=\left(g_{1} p g_{2}^{-1}, g_{2}\right. \\
\text { where } \star_{1} \text { is the action of } \operatorname{SU}(2) \text { or }\end{array}$ \\
\hline $\begin{array}{l}Q_{B}^{7} \\
\text { (all) }\end{array}$ & $S^{3} \times S^{3}$ on $S^{3} \times S^{4}$ & $\begin{array}{l}-\times \mathbb{R}) \\
\operatorname{via}\left(g_{1}, g_{2}\right) \star(p, q, t)=\left(g_{1} p g_{2}^{-1}, g_{2} q, t\right)\end{array}$ \\
\hline $\begin{array}{l}Q_{C}^{7} \\
\text { (all) }\end{array}$ & $\begin{array}{r}S^{3} \times S^{3} \times S^{1} \text { on } S^{3} \times \\
\mathrm{v}\end{array}$ & $\begin{array}{l}\times(\operatorname{Im}(\mathbb{M}) \times \mathbb{C}) \\
2, z) \star(p, q, w)=\left(g_{1} p \bar{z}^{n}, g_{2} q g_{2}^{-1}, z^{m} w\right)\end{array}$ \\
\hline $\begin{array}{l}Q_{D}^{7} \\
\text { part) }\end{array}$ & $\begin{array}{r}S^{3} \times S^{3} \times S^{1} \text { on } S^{3} \times \\
\text { vi }\end{array}$ & $\begin{array}{l}\times(\operatorname{Im}(\mathbb{I}) \times \mathbb{C}) \\
, z) \star(p, q, w)=\left(g_{1} p g_{2}^{-1}, g_{2} q g_{2}^{-1}, z w\right)\end{array}$ \\
\hline $\begin{array}{l}N_{F}^{7} \\
\text { part) }\end{array}$ & $S^{3} \times S^{3} \times S^{1}$ on $S^{2} \times$ & $\begin{array}{l}(\mathbb{W}) \times(\mathbb{H} \times \mathbb{C}) \\
, z) \star(p, q, w)=\left(g_{1} p g_{1}^{-1}, g_{1} q g_{2}^{-1}, z w\right)\end{array}$ \\
\hline $\begin{array}{l}Q_{F}^{7} \\
\text { (all) }\end{array}$ & $\mathrm{SU}(3) \times S^{1}$ on $S^{2} \times S$ & $\begin{array}{l}(\mathbb{U}) \times \mathbb{C}^{3} \\
\quad \operatorname{via}(A, z) \star(p, x)=\left(z^{n} p \bar{z}^{n}, z^{m} A x\right)\end{array}$ \\
\hline
\end{tabular}

Table VIII. Nonreducible isometric cohomogeneity one actions on compact simply connected products of irreducible symmetric spaces in dimensions 5, 6 and 7; these are not product actions. Also indicated is whether the family of actions listed in the right column makes up all or part of the family listed in the left. (Sequel to Table VII.)

$P^{5}$ : One example of this family is the usual $\mathrm{S}(\mathrm{U}(2) \mathrm{U}(1)) \subset \mathrm{SU}(3)$ action on $\mathrm{SU}(3) / \mathrm{SO}(3)$. This gives $P^{5}$ in the case $p=1$. In Section 7 we will show that $P^{5}$ is in fact always diffeomorphic to $\mathrm{SU}(3) / \mathrm{SO}(3)$.

$P_{A}^{7}$ : This family is interesting because of its similarity to the families $P_{B}^{7}$ and $P_{C}^{7}$. One very special case of this family is the action of $S^{3} \times S^{3}$ on $S^{3} \times \mathbb{C P}^{2}$ given by $\left(g_{1}, g_{2}\right) \star(p, x)=\left(g_{1} p g_{2}^{-1}, g_{2} \star_{1} x\right)$, where $\star_{1}$ is the action of $\mathrm{SO}(3)$ on $\mathbb{C P}^{2}$, which corresponds to the case $p_{-}=q_{-}=p_{+}=q_{+}=1$.

$P_{B}^{7}$ and $P_{C}^{7}$ : Grove, Wilking and Ziller [2008] computed the homology groups of these families. They showed that these two classes contain all the new candidates for compact simply connected cohomogeneity one manifolds with invariant metrics of positive curvature, with one exception. Grove and Ziller [2000] showed that the class $P_{C}^{7}$ contains all $S^{3}$ principal bundles over $S^{4}$. Two explicit actions of type $P_{C}^{7}$ are the isometric actions on $S^{7}$ and on $S^{3} \times S^{4}$ listed 
in Tables VI and VIII, respectively. An example of the family $P_{B}^{7}$ is the action of $\mathrm{SO}(3) \times \mathrm{SO}(3)$ on the Aloff-Wallach space $W^{7}=\mathrm{SU}(3) / \operatorname{diag}\left(z, z, \bar{z}^{2}\right)$, as described in [Grove et al. 2008, Section 4].

$P_{D}^{7}$ : This family contains the cohomogeneity one Eschenburg spaces

$$
E_{p}^{7}=\operatorname{diag}\left(z, z, z^{p}\right) \backslash \mathrm{SU}(3) / \operatorname{diag}\left(1,1, \bar{z}^{p+2}\right),
$$

where $\mathrm{SU}(2) \times \mathrm{SU}(2)$ acts on $E_{p}^{7}$ with the first factor acting on the left and the second on the right, both as the upper SU(2) block in SU(3). These actions correspond to the case $n=2$ and $(p, q)=(p, p+1)$ in the family $P_{D}^{7}$. It should be noted that all of these Eschenburg spaces admit invariant metrics of positive sectional curvature, by [Eschenburg 1984]. For details, see [Grove et al. 2008].

The action on $S^{3} \times \mathbb{C} \mathbb{P}^{2}$ given in Table VIII is another example of type $P_{D}^{7}$, this time with $n=1$ and $p=q=1$.

Nonprimitive actions of Table II. Recall from Proposition 1.5 that for a nonprimitive action of $G$ on $M_{G}$, with $G \supset L \supset K^{-}, K^{+} \supset H$, we have the fiber bundle $M_{L} \rightarrow M_{G} \rightarrow G / L$, where $M_{L}$ is the cohomogeneity one manifold given by the diagram $L \supset K^{-}, K^{+} \supset H$. Having such a fiber bundle says something about the topology of the manifold $M_{G}$. Because of this, we will list these bundles below. For more details about how we get the specific fiber bundles below, see Section 6 . $N^{5}$ : We saw in Section 5.2 that the Brieskorn varieties for $d$ even are all examples of this family. There is one more explicit action that is also of this type. Let $S^{3} \times S^{1}$ act on $S^{3} \times S^{2} \subset \mathbb{} \times \operatorname{Im} \mathbb{v}$ via $\left(g, e^{i \theta}\right) \star(q, v)=\left(g q \bar{g} e^{g v \bar{g} \theta}, g v \bar{g}\right)$. Here, we are using the notation $e^{x \theta}=\cos \theta+x \sin \theta \in S^{3}$ for $x \in S^{3} \cap \operatorname{Im}(\mathbb{M})$, so for example $g e^{x \theta} \bar{g}=e^{g x \bar{g} \theta}$. This action gives the diagram $S^{3} \times S^{1} \supset\left\{\left(e^{i \theta}, 1\right)\right\},\left\{\left(e^{i \theta}, e^{2 i \theta}\right)\right\} \supset\{( \pm 1,1)\}$, which is a special case of $N^{5}$. In Section 7, we will show every manifold of this type is either $S^{3} \times S^{2}$ or the nontrivial $S^{3}$ bundle over $S^{2}$.

$N_{A}^{6}$ : Consider the family of actions of $S^{3} \times S^{1} \times S^{1}$ on $S^{3} \times S^{3}$ given by $(g, z, w) \star$ $(x, y)=\left(g x \bar{z}^{r} \bar{w}^{s},\left(z^{c_{-}} \bar{w}^{b_{-}}, z^{c_{+}} \bar{w}^{b_{+}}\right) \star_{1} y\right)$, where $\star_{1}$ is the usual torus action on $S^{3} \subset \mathbb{C}^{2}$. This action gives the diagram

$$
\begin{aligned}
S^{3} \times T^{2} \supset\left\{\left(z^{r} w^{s}, z, w\right) \mid z^{c_{-}} \bar{w}^{b_{-}}=1\right\},\left\{\left(z^{r} w^{s}, z, w\right) \mid z^{c_{+}} \bar{w}^{b_{+}}=1\right\} \supset \\
\left\{\left(z^{r} w^{s}, z, w\right) \mid z^{c_{-}} \bar{w}^{b_{-}}=1=z^{c_{+}} \bar{w}^{b_{+}}\right\},
\end{aligned}
$$

which is an action of type $N_{A}^{6}$. In fact, it was shown in [Hoelscher 2010a] that every action of type $N_{A}^{6}$ can be obtained in this way. Therefore the family $N_{A}^{6}$ consists entirely of isometric actions on $S^{3} \times S^{3}$.

\footnotetext{
${ }^{1}$ In particular, $N_{A}^{6}$ could have been left out of Table II entirely; however I did not know this when first making the table. The notation has already been cited in the literature so I have left Table II unchanged.
} 
$N_{B}^{6}$ : Here, if we take $L=T^{2}$ we get the fiber bundle $S^{2} \rightarrow M \rightarrow S^{2} \times S^{2}$ for any $M$ in the family $N_{B}^{6}$. If $p=0$ in this family, we get the product action on $S^{2} \times M^{4}$, where $M^{4}$ is either $S^{2} \times S^{2}$ or $\mathbb{C P}^{2} \#-\mathbb{C P}^{2}$, depending on whether $n$ is even or odd, respectively (see [Parker 1986]).

$N_{C}^{6}$ : For each $M$ of this type, we can take $L=S^{3} \times S^{1}$ to get the fiber bundle $S^{4} \rightarrow M \rightarrow S^{2}$.

$N_{D}^{6}$ : If we let $L=S^{3} \times S^{1}$ in this case too, we get the fiber bundle $\mathbb{C P}^{2} \rightarrow M \rightarrow S^{2}$ for manifolds $M$ of this type. If $p=0, N_{D}^{6}$ is the product action on $\mathbb{C P}^{2} \times S^{2}$.

$N_{E}^{6}$ : In the case that $p=1$ we get the $S^{3} \times S^{3}$ action on $S^{2} \times S^{4} \subset \operatorname{Im} \mathbb{H} \times(\mathbb{H} \times \mathbb{R})$ given by $\left(g_{1}, g_{2}\right) \star(x,(y, t))=\left(g_{1} x g_{1}^{-1},\left(g_{1} y g_{2}^{-1}, t\right)\right)$. In this case we get the diagram

$$
S^{3} \times S^{3} \supset S^{3} \times S^{1}, S^{3} \times S^{1} \supset \Delta S^{1} .
$$

Similarly, when $p=0$ this is a product action on $S^{2} \times S^{4}$.

For a general $M$ in this family, if we take $L=S^{3} \times S^{1}$ we get the nonprimitivity fiber bundle $S^{4} \rightarrow M \rightarrow S^{2}$.

$N_{F}^{6}$ : One special case of this class of actions is the $\mathrm{SU}(3)$ action on $\mathbb{C P}^{3} \#-\mathbb{C P}^{3}$ obtained by gluing two copies of the $\mathrm{SU}(3)$ action on $\mathbb{C P}^{3}$ along the fixed point. We get

$$
\mathrm{SU}(3) \supset \mathrm{S}(\mathrm{U}(2) \mathrm{U}(1)), \mathrm{S}(\mathrm{U}(2) \mathrm{U}(1)) \supset \mathrm{SU}(2) \mathrm{SU}(1) \text {. }
$$

In general, for any $M$ in this family, we can take $L=\mathrm{S}(\mathrm{U}(2) \times \mathrm{U}(1))$ and get the fiber bundle $S^{2} \rightarrow M \rightarrow \mathbb{C} \mathbb{P}^{2}$.

$N_{A}^{7}$ : One special case of this family is the $S^{3} \times S^{3}$ action on $S^{2} \times S^{2} \times S^{3} \subset$ $\operatorname{Im}(\mathbb{H}) \times \operatorname{Im}(\mathbb{H}) \times \mathbb{U}$ via $\left(g_{1}, g_{2}\right) \star\left(p_{1}, p_{2}, q\right)=\left(g_{1} p_{1} g_{1}^{-1}, g_{1} p_{2} g_{1}^{-1}, g_{1} q g_{2}^{-1}\right)$, or the equivalent action of $\left(g_{1}, g_{2}\right) \star\left(p_{1}, p_{2}, q\right)=\left(g_{1} p_{1} g_{1}^{-1}, g_{2} p_{2} g_{2}^{-1}, g_{1} q g_{2}^{-1}\right)$. Also, the case $\left(p_{ \pm}, q_{ \pm}\right)=(0,1)$ and $H=\mathbb{Z}_{n}$ gives the product action on $S^{3} \times M^{4}$, where $M^{4}$ is either $\mathbb{C} \mathbb{P}^{2} \#-\mathbb{C P}^{2}$ or $S^{2} \times S^{2}$ depending on whether $n$ is odd or even.

For this family, the nonprimitivity fiber bundle depends heavily on the parameters of the action. For any $M$ in the family $N_{A}^{7}$ corresponding to a diagram with $K^{-} \neq K^{+}$, we can take $L=T^{2}$ to get the fiber bundle $L_{m}(n) \rightarrow M \rightarrow S^{2} \times S^{2}$, where $L_{m}(n)$ is some lens space that depends on the parameters of $M$ in $N_{A}^{7}$. If $M$ is a member of this family with $K^{-}=K^{+}$, then taking $L=T^{2}$ gives the fiber bundle $S^{2} \times S^{1} \rightarrow M \rightarrow S^{2} \times S^{2}$, and taking $L=K^{-}=K^{+}$in this case gives the bundle $S^{2} \rightarrow M \rightarrow S^{3} \times S^{2}$.

$N_{B}^{7}$ : For each $M$ in this family, we can take $L=S^{3} \times S^{1}$ to get the fiber bundle $M_{L} \rightarrow M \rightarrow S^{2}$, where $M_{L}$ is the cohomogeneity one manifold given by the diagram

$$
S^{3} \times S^{1} \supset\left\{\left(e^{i p \theta}, e^{i q \theta}\right)\right\} \cdot H,\left\{\left(e^{j \theta}, 1\right)\right\} \cdot H \supset H
$$


with the same restrictions on $H$ as in $N_{B}^{7}$. The manifolds $M_{L}$ will depend greatly on the parameters in the diagram. For example the actions $Q_{B}^{5}$ and $P^{5}$, the Brieskorn actions and the family of actions on $\mathrm{SU}(3) / \mathrm{SO}(3)$ respectively, as described in Sections 5.2 and 7.4 respectively, are both actions of this type. In fact these are the only cases when $M_{L}$ will be simply connected, assuming $p \neq 0$. Of course if $p=0$ then the original action would be of type $N_{A}^{7}$.

In the case $q=0$, the action $N_{B}^{7}$ becomes the product action on $S^{4} \times S^{3}$ or on $\mathbb{C} \mathbb{P}^{2} \times S^{3}$, depending on $H$.

$N_{C}^{7}$ : For a manifold $M$ of this type, we can take $L=S^{3} \times S^{1}$ to get the fiber bundle $S^{5} / \mathbb{Z}_{q} \rightarrow M \rightarrow S^{2}$ if $q \neq 0$, where $S^{5} / \mathbb{Z}_{q}$ is the lens space, as described in Section 6. If $q=0$ then the original action is just a product action on $\mathbb{C} \mathbb{P}^{2} \times S^{3}$.

$N_{D}^{7}$ : In this case we can take $L=T^{2}=K^{+}=K^{-}$to get the fiber bundle $S^{2} \rightarrow$ $M \rightarrow S^{3} \times S^{2}$ for any $M$ in this family. We can also take $L=T^{3} \subset S^{3} \times S^{3} \times S^{1}$ to get the bundle $S^{2} \times S^{1} \rightarrow M \rightarrow S^{2} \times S^{2}$ for any such $M$.

If $q=\lambda=0$, then this action is the product action on $S^{2} \times Q_{A}^{5}$, where $Q_{A}^{5}$ is the family of actions on $S^{2} \times S^{3}$ described above. Also if $m=0$, this is another product action on $S^{3} \times S^{2} \times S^{2}$, since it is known that $S^{3} \times S^{3} /\left\{\left(z^{p}, z^{q}\right)\right\}=S^{3} \times S^{2}$; see [Wang and Ziller 1990].

$N_{E}^{7}$ : For each $M$ in this family, let $L=T^{3} \subset S^{3} \times S^{3} \times S^{1}$. This gives the fiber bundle $L_{a}(b) \rightarrow M \rightarrow S^{2} \times S^{2}$, where $L_{a}(b)$ is some lens space that depends on the parameters of $M$ in $N_{E}^{7}$ in a complicated way.

As in the previous case, if we take $q=\lambda=0$ we get a product action on $S^{2} \times N^{5}$. Later, we will show that the actions of type $N^{5}$ are always on $S^{3} \times S^{2}$ or the nontrivial $S^{3}$ bundle over $S^{2}$.

$N_{F}^{7}$ : One example of this family is the action of $S^{3} \times S^{3} \times S^{1}$ on $S^{2} \times S^{5} \subset$ $\operatorname{Im}(\mathbb{H}) \times(\mathbb{H} \times \mathbb{C})$ given by $\left(g_{1}, g_{2}, z\right) \star(p, q, w)=\left(g_{1} p g_{1}^{-1}, g_{1} q g_{2}^{-1}, z w\right)$. Also, in the case $p=0$, we get the product action on $S^{2} \times Q_{C}^{5}$, where $Q_{C}^{5}$ is an action on $S^{5}$.

For a general $M$ in this family, taking $L=S^{3} \times S^{1} \times S^{1}$ gives the fiber bundle $S^{5} \rightarrow M \rightarrow S^{2}$.

$N_{G}^{7}$ : For this manifold, we have the bundle $S^{3} \rightarrow N_{G}^{7} \rightarrow \mathbb{C P}^{2}$, after taking $L=$ $U(2)=K^{ \pm}$.

$N_{H}^{7}$ : In this case, if we take $L=\mathrm{U}(2) \times S^{1} \subset \mathrm{SU}(3) \times S^{1}$ we get the fiber bundle $L_{a}(b) \rightarrow M \rightarrow \mathbb{C} \mathbb{P}^{2}$ for each $M$ in this family, where $L_{a}(b)$ is some lens space that depends on the parameters of $M$ in the class $N_{H}^{7}$.

$N_{I}^{7}$ : Finally, we have the fiber bundle $S^{3} \rightarrow N_{I}^{7} \rightarrow S^{4}$ for this manifold after taking $L=\operatorname{Sp}(1) \operatorname{Sp}(1)=K^{ \pm}$. 


\section{Curvature properties}

Here we will prove Theorem B. First, we have shown, through the classification, that every nonreducible cohomogeneity one action on a simply connected manifold in dimension 5, 6 or 7 must be a product action, a sum action, a fixed-point action or one of the actions found in the classification above. We know from Section 1.21 that sum actions and fixed-point actions are isometric actions on symmetric spaces, and hence they admit invariant metrics of nonnegative curvature. For product actions, let $M=N \times L / J$, where $N$ is a lower-dimensional cohomogeneity one manifold, $L / J$ is a homogeneous space, and $G=G_{1} \times L$ acts as a product. It is clear that the action of $G_{1}$ on $N$ is nonreducible if and only if the original action of $G$ on $M$ is. Further, $L / J$ admits an $L$ invariant metric of nonnegative sectional curvature, so if $N$ admits a $G_{1}$-invariant metric of nonnegative sectional curvature, $M=N \times L / J$ also has a $G$-invariant metric of nonnegative curvature from the product. To see that $N$ has such a metric in lower dimensions, recall from the classification of cohomogeneity one manifolds in dimensions 4 and lower [Parker 1986; Neumann 1968] that every nonreducible compact simply connected cohomogeneity one manifold in these dimensions is an isometric action on a symmetric space, with one exception: the manifolds given by the diagram $S^{3} \supset S^{1}, S^{1} \supset \mathbb{Z}_{n}$. However, these manifolds admit invariant metrics of nonnegative sectional curvature by the main result in [Grove and Ziller 2000]. Therefore, all our nonreducible product actions will admit $G$ invariant metrics of nonnegative sectional curvature.

Therefore we must only check that the remaining actions admit invariant metrics of nonnegative sectional curvature, except for the two exceptional families $P_{D}^{7}$ and $Q_{D}^{7}$ listed in Theorem B. Section 5.1 shows that many of these actions are isometric actions on symmetric spaces, and hence admit invariant metrics of nonnegative curvature. Many of the actions also have codimension two singular orbits. Therefore, by the main result in [Grove and Ziller 2000], these also admit invariant nonnegative curvature. After these two considerations, we are only left with these actions to check: $N_{C}^{6}, N_{D}^{6}, N_{E}^{6}, N_{C}^{7}, N_{F}^{7}, N_{G}^{7}$ and $N_{I}^{7}$.

These seven actions are all nonprimitive. We will use Proposition 1.5 to write each manifold $M$ of these types in the form $G \times{ }_{L} M_{L}$, and in each case we will see that the $L$ action on $M_{L}$ admits an invariant metric of nonnegative sectional curvature. This will show that $M \approx G \times{ }_{L} M_{L}$ admits a $G$ invariant metric of nonnegative sectional curvature, since we can take the metric mentioned above on $M_{L}$ and a biinvariant metric on $G$ to induce a submersed metric on $M$. This metric will still be nonnegatively curved by O'Niell's formula; see [Petersen 1998]. We will proceed to do this case by case.

In the case of $N_{C}^{6}$, the subdiagram corresponding to $M_{L}$ is given by $\left(N_{C}^{6}\right)$, but with $S^{3} \times S^{1}$ in place of $S^{3} \times S^{3}$. This action is ineffective, with the effective version 
given by taking $n=1$. We then recognize this effective version as an isometric sum action on $S^{4}$. Therefore $M_{L}$ admits an $L$ invariant metric of nonnegative sectional curvature.

We can do a similar thing for actions $N_{D}^{6}$ and $N_{E}^{6}$. The primitive subdiagram for $N_{D}^{6}$ is given by $\left(N_{D}^{6}\right)$, but with $S^{3} \times S^{1}$ in place of $S^{3} \times S^{3}$. This is the action of $\mathrm{SU}(2) \times S^{1}$ on $\mathbb{C} \mathbb{P}^{2}$ given by $(A, w) \star\left[z_{1}, z_{2}, z_{3}\right]=\left[w^{p} z_{1}, A\left(z_{2}, z_{3}\right)^{t}\right]$. Since this is an isometric action for the usual metric on $M_{L}=\mathbb{C P}^{2}$, this gives an invariant metric on $M \approx G \times{ }_{L} M_{L}$. Similarly the primitive subdiagram for $N_{E}^{6}$ is $\left(N_{E}^{6}\right)$, but with $S^{3} \times S^{1}$ in place of $S^{3} \times S^{3}$. This is the action of $S^{3} \times S^{1}$ on $S^{4} \subset \mathbb{M} \times \mathbb{R}$ by $(g, z) \star(p, t)=\left(g p \bar{z}^{p}, t\right)$. As above this also gives $N_{E}^{6}$ an invariant metric of nonnegative sectional curvature.

For $N_{C}^{7}$, the primitive subdiagram is given by $\left(N_{C}^{7}\right)$, but with $S^{3} \times S^{1}$ in place of $S^{3} \times S^{3}$, where $\operatorname{gcd}(p, q)=1$ and $\operatorname{gcd}(q, n)=1$. We claim this is an isometric action on the lens space $S^{5} / \mathbb{Z}_{q}$. It is easy to check that the special case of this action, when $q=1$, is the modified sum action of SU(2) $\times S^{1}$ on $S^{5} \subset \mathbb{C}^{2} \times \mathbb{C}$ by $(A, w) \star(x, z)=\left(w^{p} A x, w^{n} z\right)$. Then consider $S^{5} / \mathbb{Z}_{q}$, where $\mathbb{Z}_{q}$ acts as $\mathbb{Z}_{q} \subset 1 \times \mathbb{Z}_{q}$ with this same action, $\star$. Then $\mathrm{SU}(2) \times S^{1}$ still acts on the quotient $S^{5} / \mathbb{Z}_{q}$ and does so isometrically in the induced metric. If the original group diagram is taken along the geodesic $c$ in $S^{5}$ then we can take the group diagram of the induced action on $S^{5} / \mathbb{Z}_{q}$ along the image of $c$. When we do this we see we get exactly the diagram shown above. Hence this is an isometric action on $S^{5} / \mathbb{Z}_{q}$ in the usual, positively curved, metric. As above, this induces an invariant metric of nonnegative sectional curvature on $N_{C}^{7}$.

The last three cases are slightly easier to handle. For $N_{F}^{7}$ the primitive subdiagram is given by

$$
S^{3} \times S^{1} \times S^{1} \supset\left\{\left(e^{i p \phi} e^{i a \theta}, e^{i \phi}, e^{i \theta}\right)\right\}, S^{3} \times S^{1} \times \mathbb{Z}_{n} \supset\left\{\left(e^{i p \phi}, e^{i \phi}, 1\right)\right\} \cdot \mathbb{Z}_{n} .
$$

We see this is the modified sum action of $S^{3} \times S^{1} \times S^{1}$ on $S^{5} \subset \mathbb{M} \times \mathbb{C}$ given by $\left(g, z_{1}, z_{2}\right) \star(y, w)=\left(g y \bar{z}_{1}^{p} \bar{z}_{2}^{a}, z_{2}^{n} w\right)$. Similarly the primitive subdiagram for $N_{G}^{7}$ is given by the action of $\mathrm{U}(2)$ on $\mathrm{SU}(2) \approx S^{3}$ by conjugation and the subdiagram for $N_{I}^{7}$ corresponds to the action of $\mathrm{Sp}(1)$ on $\mathrm{Sp}(1)$ by conjugation. Both of these are isometries in the positively curved biinvariant metric on $\mathrm{Sp}(1) \approx \mathrm{SU}(2) \approx S^{3}$. Therefore these seven remaining cases do admit invariant metrics of nonnegative sectional curvature. This proves Theorem B.

\section{Topology of the 5-dimensional manifolds}

In this section we will determine the diffeomorphism type of the five-dimensional manifolds appearing in the classification and prove Theorem C. By the results of Smale and Barden [Barden 1965], the diffeomorphism type of a closed simply 
connected 5-manifold is determined by the second homology group and the second Stiefel-Whitney class. As we will see, we can compute the homology of our manifolds relatively easily. To compute the second Stiefel-Whitney class, we will use the topology of the frame bundle. Recall that the second Stiefel-Whitney class of a simply connected manifold is zero if and only if the manifold is a Spin-manifold, that is, the orthonormal frame bundle lifts to a Spin-bundle; see [Petersen 1998]. With this motivation, we will now look at the frame bundle in more detail.

Suppose $M^{n}$ is an oriented cohomogeneity one manifold with the group diagram $G \supset K^{-}, K^{+} \supset H$ as usual. Assume further that $G$ is connected so that the $G$ action preserves the orientation of $M$. Then let

$F M=\left\{f=\left(f_{1}, \ldots, f_{n}\right) \mid f_{1}, \ldots, f_{n}\right.$ is an oriented orthonormal frame at $\left.p \in M\right\}$

denote the orthonormal oriented-frame bundle of $M$. Recall that $\mathrm{SO}(n)$ acts on $F M$ from the left as

$$
\left(a_{i j}\right)_{i j} \star\left(f_{1}, \ldots, f_{n}\right)=\left(\sum_{j} a_{1 j} f_{j}, \ldots, \sum_{j} a_{n j} f_{j}\right) .
$$

This action makes $F M$ into an $\mathrm{SO}(n)$-principal bundle over $M$. We can put a metric on $F M$ by choosing a biinvariant metric on $\mathrm{SO}(n)$, keeping the original metric on $M$ and specifying a horizontal distribution. To describe this distribution, fix a point $p_{0} \in M$ and a frame $f_{p_{0}}$ at $p_{0}$. For each $p$ in a normal neighborhood of $p_{0}$, let $f_{p}$ be the frame gotten by parallel translating $f_{p_{0}}$ to $p$ along the minimal geodesic from $p_{0}$ to $p$. This gives a local orthonormal frame field, and we define the horizontal space at $f_{p_{0}} \in F M$ to be the tangent space of this frame field. Since parallel transport is an isometry, the action of $\mathrm{SO}(n)$ preserves this horizontal distribution.

Recall that $G$ acts on $M$ by isometry and hence takes orthonormal frames to orthonormal frames, while preserving orientation. Therefore we have an induced action of $G$ on $F M$ given by $g \star(p, f)=(g p, d g f)=\left(g p,\left(d g f_{1}, \ldots, d g f_{n}\right)\right)$. This action is isometric since it takes the horizontal space to the horizontal space and acts by isometry on both the vertical and horizontal spaces. This $G$-action also commutes with the action by $\mathrm{SO}(n)$, and so we have an action by $G \times \mathrm{SO}(n)$ on $F M$. Furthermore, this $G \times \mathrm{SO}(n)$ action on $F M$ is clearly cohomogeneity one since $\mathrm{SO}(n)$ acts transitively on the fibers of $F M$. If $c$ denotes a minimal geodesic in $M$ between nonprincipal orbits, then choose $f(t)$ to be a parallel orthonormal frame along $c$. Then $f$ is a horizontal curve in $F M$ and therefore a geodesic. $f(t)$ is clearly perpendicular to the $\mathrm{SO}(n)$ orbits, and it is perpendicular to the $G$ orbits since $c(t)$ is in $M$. Therefore $f(t)$ is a minimal geodesic in $F M$ between nonprincipal orbits.

Our next goal is to determine the isotropy groups of $G \times \mathrm{SO}(n)$ along $f(t)$. We see $(g, A) \star(p, f)=(p, f)$ if and only if $g \in G_{p}$ and $A \star d g f=f$, where $\star$ is 
from (7-1). To understand this second equality we rewrite it as

$$
\left(d g^{-1} f_{1}, \ldots, d g^{-1} f_{n}\right)=d g^{-1} f=A \star f=\left(\sum_{j} a_{1 j} f_{j}, \ldots, \sum_{j} a_{n j} f_{j}\right) .
$$

This precisely means $A^{t}=\left[d g^{-1}\right]_{f}$ or $A=[d g]_{f}$ where $[d g]_{f}$ is the representation of the linear operator $d g: T_{p} M \rightarrow T_{p} M$ as a matrix in the basis $f_{1}, \ldots, f_{n}$. In conclusion, the isotropy group of $G \times \mathrm{SO}(n)$ at $(p, f)$ is $\left\{\left(g,[d g]_{f}\right) \mid g \in G_{p}\right\}$. We have proved the following proposition.

Proposition 7.1. Let $M^{n}$ be an oriented cohomogeneity one manifold with group diagram $G \supset K^{-}, K^{+} \supset H$ for the normal geodesic c, and assume $G$ is connected. The orthonormal oriented frame bundle FM of $M$ admits a natural cohomogeneity one action with group diagram

$$
\begin{array}{r}
G \times \operatorname{SO}(n) \supset\left\{\left(k,[d k]_{f(-1)}\right) \mid k \in K^{-}\right\},\left\{\left(k,[d k]_{f(1)}\right) \mid k \in K^{+}\right\} \\
\\
\supset\left\{\left(h,[d h]_{f(0)}\right) \mid h \in H\right\},
\end{array}
$$

where $f(t)$ is a parallel frame along $c(t)$.

Corollary 7.2. Let $M$ be a cohomogeneity one manifold as in Proposition 7.1 and assume that $H$ is discrete. Let $\alpha_{ \pm}:[0,1] \rightarrow K^{ \pm}$be paths, based at the identity, that generate $\pi_{1}\left(K^{ \pm} / H\right)$. If $M$ is simply connected, then FM is simply connected if and only if there is some curve $\gamma=\alpha_{-}^{n} \cdot \alpha_{+}^{m}$ that gives a contractible loop in $G$ and where $\left[d \alpha_{-}^{n}\right]_{f(-1)} \cdot\left[d \alpha_{+}^{m}\right]_{f(1)}$ generates $\pi_{1}(\mathrm{SO}(n))$.

Proof. Notice that the maps $k \mapsto\left(k,[d k]_{f( \pm 1)}\right)$ give isomorphisms of $K^{ \pm}$with $\hat{K}^{ \pm}:=\left\{\left(k,[d k]_{f( \pm 1)}\right) \mid k \in K^{ \pm}\right\}$, the nonprincipal isotropy subgroups of the $\hat{G}:=$ $G \times \mathrm{SO}(n)$ action on $F M$. Also, this map takes $H$ to $\hat{H}:=\left\{\left(h,[d h]_{f(0)}\right) \mid h \in H\right\}$, the principal isotropy group of this action. Thus we see that $\hat{H}$ is generated by $\hat{H} \cap \hat{K}_{0}^{-}$and $\hat{H} \cap \hat{K}_{0}^{+}$by Lemma 1.10 , since $M$ is already assumed to be simply connected. Then by Lemma 1.10, FM is simply connected if and only if the curves $\hat{\alpha}_{ \pm}(t)=\left(\alpha_{ \pm}(t),\left[d \alpha_{ \pm}(t)\right]_{f( \pm 1)}\right)$ generate $\pi_{1}\left(\hat{G} / \hat{H}_{0}\right)$. Further, since $H$ is discrete, $\pi_{1}\left(\hat{G} / \hat{H}_{0}\right)=\pi_{1}(\hat{G}) \approx \pi_{1}(G) \times \pi_{1}(\mathrm{SO}(n))$.

Therefore, if $\pi_{1}(F M)=0$ then the claimed curve $\gamma$ must exist. Conversely, suppose such a curve $\gamma$ exists. We already know from Lemma 1.10 that $\alpha_{-}$and $\alpha_{+}$ generate $\pi_{1}(G)$, since $M$ is simply connected. Then it is clear that $\hat{\alpha}_{-}, \hat{\alpha}_{+}$and $\gamma$ would generate all of $\pi_{1}(\hat{G})$, proving $\pi_{1}(F M)=0$.

7.3. The family $\boldsymbol{P}^{\mathbf{5}}$. We will now compute the homology of the manifolds $P^{5}$ using the Mayer-Vietoris sequence. As in the proof of Proposition 1.8, we have the Mayer-Vietoris sequence for the spaces $M$, with $G / K^{-}, G / K^{+}$and $G / H$ as follows.

$$
\cdots \rightarrow H_{n}(G / H) \stackrel{\left(\rho_{*}^{-}, \rho_{*}^{+}\right)}{\longrightarrow} H_{n}\left(G / K^{-}\right) \oplus H_{n}\left(G / K^{+}\right) \stackrel{i_{*}^{-}-i_{*}^{+}}{\longrightarrow} H_{n}(M) \rightarrow \cdots
$$


To compute $H_{n}\left(P^{5}\right)$ in our case, first note that $G / K^{+}=S^{3} \times S^{1} /\left\{\left(e^{j p \theta}, e^{i \theta}\right)\right\} \approx S^{3}$, since $S^{3}$ acts transitively on this space with trivial isotropy group.

Next we claim that $G / H=S^{3} \times S^{1} /\langle(j, i)\rangle$ is $S^{3} \times S^{1}$. For this, denote

$$
\alpha: S^{3} \times S^{1} \rightarrow S^{3} \times S^{1}, \quad(g, z) \mapsto(g j, z i),
$$

so that $G / H=G /\langle\alpha\rangle$. Then define the map

$$
\phi: S^{3} \times S^{1} \rightarrow S^{3} \times S^{1}, \quad\left(g, e^{i \theta}\right) \mapsto\left(g e^{-j \theta}, e^{i \theta}\right),
$$

a diffeomorphism of manifolds. We notice that $\beta:=\phi \alpha \phi^{-1}:(g, z) \mapsto(g, z i)$. Therefore $G /\langle\alpha\rangle$ is diffeomorphic to $G /\langle\beta\rangle \approx S^{3} \times S^{1}$.

Finally we study $G / K^{-}=S^{3} \times S^{1} /\left\{\left(e^{i \theta}, 1\right)\right\} \cdot\langle(j, i)\rangle$. Since $K_{0}^{-}$is normal in $K^{-}$, we have $G / K^{-} \approx\left(G / K_{0}^{-}\right) /\left(K^{-} / K_{0}^{-}\right)$. We see $G / K_{0}^{-}=S^{3} \times S^{1} /\left\{\left(e^{i \theta}, 1\right)\right\} \approx$ $S^{2} \times S^{1}=\operatorname{Im}\left(S^{3}\right) \times S^{1}$ via $\left(g S^{1}, z\right) \mapsto\left(g i g^{-1}, z\right)$. Further, it is easy to see that $(j, i)$ acts on $S^{2} \times S^{1}$ as $(-\mathrm{Id}, i)$, via this correspondence. Therefore we have $G / K^{-} \approx S^{2} \times S^{1} /\langle(-\mathrm{Id}, i)\rangle$. We can identify this space with $S^{2} \times[0,1] / \sim$ where $(x, 0) \sim(-x, 1)$. Using Mayer-Vietoris for this space, we can easily compute that $H_{i}\left(G / K^{-}\right)$is equal to $\mathbb{Z}$ if $i=1$, to $\mathbb{Z}_{2}$ if $i=2$, and to 0 otherwise.

We are now ready to use the Mayer-Vietoris sequence for $P^{5}$. Equation (7-3) becomes

$$
\cdots \rightarrow 0 \rightarrow \mathbb{Z}_{2} \oplus 0 \rightarrow H_{2}\left(P^{5}\right) \rightarrow \mathbb{Z} \rightarrow \mathbb{Z} \oplus 0 \rightarrow 0 \rightarrow \cdots,
$$

since we know $H_{1}\left(P^{5}\right)=0$. Since the map $\mathbb{Z} \rightarrow \mathbb{Z} \oplus 0$ is onto, it must have trivial kernel and hence the map from $H_{2}\left(P^{5}\right)$ must be trivial. Therefore $\mathbb{Z}_{2} \oplus 0 \rightarrow H_{2}\left(P^{5}\right)$ must be an isomorphism. That is, $H_{2}\left(P^{5}\right)=\mathbb{Z}_{2}$. Poincaré duality then determines the rest of the homology groups.

To determine the second Steifel-Whitney class, we look at the fundamental group of the frame bundle $F\left(P^{5}\right)$. In the notation of Corollary 7.2, we can take $\alpha_{-}(\theta)=\left(e^{i \theta}, 1\right)$, in this case, since this is a curve in $K^{-}$that generates $\pi_{1}\left(K^{-} / H\right)$. We need to determine how $d\left(\alpha_{-}(\theta)\right)$ acts on $T_{c(-1)} M \approx T_{K^{-}}\left(G / K^{-}\right) \oplus T_{0} D_{-}$, where $D_{-}$is the disk normal to the orbit $G \cdot c(-1)$ at $c(-1)$. On $T_{K^{-}}\left(G / K^{-}\right)$, $d\left(\alpha_{-}(\theta)\right)$ has the form $\operatorname{diag}(R(2 \theta), 1)$ in the basis $\{(j, 0),(k, 0),(0,1)\}$, and since $d\left(\alpha_{-}(\theta)\right)$ is an isometry of $T_{K^{-}}\left(G / K^{-}\right)$there must be an orthonormal basis of $T_{K^{-}}\left(G / K^{-}\right)$in which $d\left(\alpha_{-}(\theta)\right)$ still has this form. Now, $d\left(\alpha_{-}(\theta)\right)$ on $D_{-}$acts isometrically as $R(\theta)$. Therefore there is an oriented orthonormal basis $f_{-}$of $T_{c(-1)} M$ for which $\left[d\left(\alpha_{-}(\theta)\right)\right]_{f_{-}}=\operatorname{diag}(R(2 \theta), 1, R(\theta))$. Since this generates $\pi_{1}(\mathrm{SO}(5))$ and since $\alpha_{-}$is contractible in $G$, it follows from Corollary 7.2 that $F\left(P^{5}\right)$ is simply connected, independent of $p$.

Therefore $P^{5}$ is not Spin and hence has nontrivial second Steifel-Whitney class for each $p$. By the results of Smale and Barden mentioned above this proves that the diffeomorphism type of $P^{5}$ is independent of $p$. In Section 5.3, we showed 
that $\mathrm{SU}(3) / \mathrm{SO}(3)$ is one example in this family. Hence $P^{5}$ is diffeomorphic to $\mathrm{SU}(3) / \mathrm{SO}(3)$ for all $p$.

7.4. The family $N^{5}$. We now compute the topology of the manifolds $N^{5}$, this time using the nonprimitive fiber bundle. Notice first that these manifolds are not primitive. In fact if we take $L=T^{2}$ then $K^{-}, K^{+}, H \subset L$. Therefore, by Proposition $1.5, N^{5}$ is fibered over $G / L \approx S^{2}$ with fiber $M_{L}$, the cohomogeneity one manifold given by

$$
S^{1} \times S^{1} \supset\left\{\left(e^{i p_{-} \theta}, e^{i q_{-} \theta}\right)\right\} \cdot H,\left\{\left(e^{i p_{+} \theta}, e^{i q_{+} \theta}\right)\right\} \cdot H \supset H_{-} \cdot H_{+} .
$$

Since $H$ is normal in $T^{2}$, it follows that the effective version of the $L$ action on $M_{L}$ is given by

$$
S^{1} \times S^{1} \supset\left\{\left(e^{i \theta}, 1\right)\right\},\left\{\left(e^{i p \theta}, e^{i q \theta}\right)\right\} \supset 1
$$

after taking an automorphism of $T^{2}$, where $q \neq 0$ since $K^{+} \neq K^{-}$in the original diagram. To identify this action first recall that $T^{2}$ acts by cohomogeneity one on $S^{3} \subset \mathbb{C}^{2}$, by multiplication on each factor. If we take $S^{3} /\left\langle\left(\xi_{q}, \xi_{q}^{p}\right)\right\rangle$, where $\xi_{q}$ is a $q$-th root of unity, this gives the lens space $L_{q}(p)$. Since the $T^{2}$ action on $S^{3}$ commutes with this subaction by $\mathbb{Z}_{q}$, we get an induced action on $L_{q}(p)$. It is not difficult to see that the effective version of this action is precisely the action given by (7-4). Therefore $M_{L}$ is $L_{q}(p)$ and we have the fibration $L_{q}(p) \rightarrow N^{5} \rightarrow S^{2}$.

Given that $N^{5}$ is simply connected, the long exact sequence of homotopy groups induced from this fibration contains the short exact sequence

$$
0 \rightarrow \pi_{2}\left(N^{5}\right) \rightarrow \pi_{2}\left(S^{2}\right) \rightarrow \pi_{1}\left(L_{q}(p)\right) \rightarrow 0 .
$$

Since the middle group is $\mathbb{Z}$ and the last group is $\mathbb{Z}_{q}$ for $q \neq 0$, it follows that $\pi_{2}\left(N^{5}\right) \approx \mathbb{Z}$ and hence $H_{2}\left(N^{5}\right) \approx \mathbb{Z}$, by the Hurewicz theorem.

We claim here that the frame bundle $F\left(N^{5}\right)$ can either be simply connected or not, depending on the parameters of the diagram. In Section 5.3, we saw one example of an action in this family on $S^{3} \times S^{2}$. This shows that some of these actions are on spin manifolds. To see that some of these manifolds are not spin we take a simple example. The manifold $M_{1}$ with group diagram

$$
S^{3} \times S^{1} \supset S^{1} \times 1,1 \times S^{1} \supset 1
$$

is an example of type $N^{5}$. If we let $\alpha_{-}(\theta)=\left(e^{i \theta}, 1\right)$, then $\alpha_{-}$generates $\pi_{1}\left(K^{-} / H\right)$. By precisely the same argument as in the case of $P^{5}$, we see that $F\left(M_{1}\right)$ is simply connected. Therefore the family $N^{5}$ contains both spin and nonspin manifolds, but always with the homology of $S^{3} \times S^{2}$. Using [Barden 1965] again, this proves Theorem C. 


\section{Acknowledgments}

This work was completed as a Ph.D. thesis at the University of Pennsylvania. I would like to extend my deepest thanks to my advisor, W. Ziller, for his tremendous help throughout the entire process. I also thank the referee for carefully reading the manuscript and for helpful suggestions.

\section{References}

[Barden 1965] D. Barden, "Simply connected five-manifolds", Ann. of Math. (2) 82 (1965), 365385. MR 32 \#1714 Zbl 0136.20602

[Besse 1978] A. L. Besse, Manifolds all of whose geodesics are closed, Ergebnisse der Mathematik und ihrer Grenzgebiete 93, Springer, Berlin, 1978. MR 80c:53044 Zbl 0387.53010

[Bredon 1972] G. E. Bredon, Introduction to compact transformation groups, Pure and Applied Mathematics 46, Academic Press, New York, 1972. MR 54 \#1265 Zbl 0246.57017

[Cleyton and Swann 2002] R. Cleyton and A. Swann, "Cohomogeneity-one $G_{2}$-structures", J. Geom. Phys. 44:2-3 (2002), 202-220. MR 2004a:53051 Zbl 1025.53024

[Conti 2007] D. Conti, "Cohomogeneity one Einstein-Sasaki 5-manifolds", Comm. Math. Phys. 274:3 (2007), 751-774. MR 2008k:53085 Zbl 1143.53041

[Cvetič et al. 2004] M. Cvetič, G. W. Gibbons, H. Lü, and C. N. Pope, "New cohomogeneity one metrics with Spin(7) holonomy", J. Geom. Phys. 49:3-4 (2004), 350-365. MR 2005f:53074 Zbl 1092.53024

[Dynkin 1952] E. B. Dynkin, "Maximal subgroups of the classical groups", Trudy Moskov. Mat. Ob̌̌č 1 (1952), 39-166. In Russian; translated in Amer. Math. Soc. Transl. (2) 6 (1957), 245-378. MR 14,244d Zbl 0077.03403

[Eschenburg 1984] J.-H. Eschenburg, Freie isometrische Aktionen auf kompakten Lie-Gruppen mit positiv gekrümmten Orbiträumen, Schriftenreihe des Mathematischen Instituts der Universität Münster (2) 32, 1984. MR 86a:53045 Zbl 0551.53024

[Escher and Ultman 2008] C. M. Escher and S. K. Ultman, "Cohomology rings of certain seven dimensional manifolds", preprint, version 2, 2008. arXiv 0810.2056v2

[Gambioli 2008] A. Gambioli, "SU(3)-manifolds of cohomogeneity one", Ann. Global Anal. Geom. 34:1 (2008), 77-100. MR 2009j:57039 Zbl 1155.57033

[Gauntlett et al. 2004] J. P. Gauntlett, D. Martelli, J. Sparks, and D. Waldram, "Sasaki-Einstein metrics on $S^{2} \times S^{3}$ ”, Adv. Theor. Math. Phys. 8:4 (2004), 711-734. MR 2006m:53067 Zbl 1136.53317

[Gibbons et al. 2004] G. W. Gibbons, S. A. Hartnoll, and Y. Yasui, "Properties of some fivedimensional Einstein metrics”, Classical Quantum Gravity 21:19 (2004), 4697-4730. MR 2006b: 53058 Zbl 1073.83035

[Grove and Ziller 2000] K. Grove and W. Ziller, "Curvature and symmetry of Milnor spheres", Ann. of Math. (2) 152:1 (2000), 331-367. MR 2001i:53047 Zbl 0991.53016

[Grove and Ziller 2002] K. Grove and W. Ziller, "Cohomogeneity one manifolds with positive Ricci curvature", Invent. Math. 149:3 (2002), 619-646. MR 2004b:53052 Zbl 1038.53034

[Grove et al. 2006] K. Grove, L. Verdiani, B. Wilking, and W. Ziller, "Non-negative curvature obstructions in cohomogeneity one and the Kervaire spheres", Ann. Sc. Norm. Super. Pisa Cl. Sci. (5) 5:2 (2006), 159-170. MR 2007h:53051 Zbl 1170.53307 
[Grove et al. 2008] K. Grove, B. Wilking, and W. Ziller, "Positively curved cohomogeneity one manifolds and 3-Sasakian geometry", J. Differential Geom. 78:1 (2008), 33-111. MR 2009m:53090 Zbl 1145.53023

[Hoelscher 2010a] C. A. Hoelscher, "Diffeomorphism type of six-dimensional cohomogeneity one manifolds", Ann. Glob. Anal. Geom. (2010).

[Hoelscher 2010b] C. A. Hoelscher, "On the homology of low-dimensional cohomogeneity one manifolds", Transformation Groups 15:1 (2010), 115-133.

[Hsiang and Lawson 1971] W.-y. Hsiang and H. B. Lawson, Jr., "Minimal submanifolds of low cohomogeneity", J. Differential Geometry 5 (1971), 1-38. MR 45 \#7645 Zbl 0219.53045

[Iwata 1981] K. Iwata, "Compact transformation groups on rational cohomology Cayley projective planes”, Tôhoku Math. J. (2) 33:4 (1981), 429-442. MR 83h:57047 Zbl 0506.57024

[Kollross 2002] A. Kollross, "A classification of hyperpolar and cohomogeneity one actions", Trans. Amer. Math. Soc. 354:2 (2002), 571-612. MR 2002g:53091 Zbl 1042.53034

[Neumann 1968] W. D. Neumann, "3-dimensional $G$-manifolds with 2-dimensional orbits", pp. 220-222 in Proc. Conf. on Transformation Groups (New Orleans, 1967), Springer, New York, 1968. MR 39 \#6355 Zbl 0177.52101

[Parker 1986] J. Parker, “4-dimensional G-manifolds with 3-dimensional orbits”, Pacific J. Math. 125:1 (1986), 187-204. MR 88e:57033 Zbl 0599.57016

[Petersen 1998] P. Petersen, Riemannian geometry, Graduate Texts in Mathematics 171, Springer, New York, 1998. MR 98m:53001 Zbl 0914.53001

[Reidegeld 2009] F. Reidegeld, "Special cohomogeneity one metrics with $Q^{111}$ or $M^{110}$ as principal orbit”, preprint, version 1, 2009. arXiv 0908.3965v1

[Straume 1996] E. Straume, Compact connected Lie transformation groups on spheres with low cohomogeneity, I, Mem. Amer. Math. Soc. 119:569, American Mathematical Society, Providence, RI, 1996. MR 96f:57036 Zbl 0854.57033

[Uchida 1977] F. Uchida, "Classification of compact transformation groups on cohomology complex projective spaces with codimension one orbits", Japan. J. Math. (N.S.) 3:1 (1977), 141-189. MR 80b:57036 Zbl 0374.57009

[Verdiani 2004] L. Verdiani, "Cohomogeneity one manifolds of even dimension with strictly positive sectional curvature", J. Differential Geom. 68:1 (2004), 31-72. MR 2006c:53033 Zbl 1100.53033

[Wang and Ziller 1990] M. Y. Wang and W. Ziller, "Einstein metrics on principal torus bundles", J. Differential Geom. 31:1 (1990), 215-248. MR 91f:53041 Zbl 0691.53036

[Ziller 1998] W. Ziller, "Homogeneous spaces, biquotients, and manifolds with positive curvature", lecture notes, 1998.

Received March 10, 2009.

\section{COREy A. HOElsCHER}

Hill AsSistant PROFESSOR

DEPARTMENT OF MATHEMATICS

RUTGERS UNIVERSITY

110 FRELINGHUYSEN ROAD

PISCATAWAY, NJ 08854-8019

UNITED STATES

coreyah@math.rutgers.edu

http://www.math.rutgers.edu/ coreyah/ 